\title{
PERIODS OF INTEGRALS ON ALGEBRAIC MANIFOLDS: SUMMARY OF MAIN RESULTS AND DISCUSSION OF OPEN PROBLEMS
}

\author{
BY PHILLIP A. GRIFFITHS
}

Table of Contents

0. Introduction .................................... 229

Part I. Summary of main results......................... 231

1. The geometric situation giving rise to variation of Hodge structure... 231

2. Data given by the variation of Hodge structure.............. 232

3. Theorems about monodromy of homology ................. 235

4. Theorems about Picard-Fuchs equations (Gauss-Manin connexion)... 237

5. Global theorems about holomorphic and locally constant cohomology

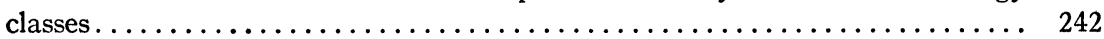

6. Global results on variation of Hodge structure............... 246

Part II. Problems and conjectures......................... 247

7. Problems on Torelli-type theorems..................... 247

8. Problems on local monodromy and variation of Hodge structure...... 248

9. Questions on compactification and the behavior of periods at infinity. . 251

AMS Subject Classifications. Primary 3260, 3265, 5366, 5380, 5760, 1401, 1455, 1449; Secondary 2065, 2257, 2270, 3247, 3250, 5550, 1418, 1420, 1451.

Key Words and Phrases. Algebraic family of algebraic varieties, smooth compactification, Gauss-Manin connexion, Hodge filtration, infinitesimal period relation, Hodge bilinear form, primitive cohomology, Lefschetz decomposition, period matrix space, period mapping, global monodromy group, monodromy theorem, PicardLefschetz transformation, Picard-Fuchs equations, regular singular points, hyperbolic complex analysis, regularity theorem, variation of Hodge structure, Hodge bundles, rigidity theorem, intermediate Jacobian, Abel-Jacobi homomorphism, generalized intermediate Jacobian, negatively curved holomorphic mappings, local Torelli problem, global Torelli problem, polarized K-3 surfaces, cubic threefolds, local invariant cycle problem, local vanishing cycle group, boundary components, parabolic subgroups, partial compactification, polarized Hodge structure, Picard-Lefschetz filtration, canonical bundle, canonical factor of automorphy, automorphic forms, automorphic cohomology, local Picard theorem, Siegel upper-half-plane, modular group, Riemann bilinear relations, Riemann extension theorem, unipotent radical, Satake topology, Siegel domain, ordinary singularities, Poincaré residue, adjoint conditions, Poincaré complete reducibility theorem, vanishing cycle, transverse cycle, intersection matrix, Hodge index theorem, Gysin homomorphism, Leray spectral sequence.

An address delivered before the 73rd Summer meeting of the Society by invitation of the Committee to Select Hour Speakers for Annual and Summer Meetings, August 29,1968 , under the title Some transcendental problems in algebraic geometry; received by the editors September 18, 1969. 
10. Problems on normalization and uniformization of periods . . . . . . 257

11. Problems on automorphic cohomology ................. 261

12. Some general problems on complex analysis and uniformization of algebraic function fields. . . . . . . . . . . . . . . . . . . . . . . . . . 265 Part III. Examples and special cases....................... 265

13. Periods of algebraic curves acquiring singularities............ 265

14. Periods of certain algebraic surfaces acquiring singularities......... 274

15. Some remarks on and examples of the local invariant cycle problem... 288 Appendix. Recent progress on the problems and conjectures .............. 293 Bibliography ........................................ 295

0. Introduction. The purpose of this paper is to give an overview of the general subject of period matrices or, what amounts to essentially the same thing, variation of Hodge structures. Our main emphasis is on the precise formulation and discussion of what I feel are the central problems and conjectures in the subject.

This paper is divided into three parts, and a short introduction to each will now be given.

(i) Part I is devoted to giving the basic framework and main results which have appeared thus far in the subject. No complete proofs are given, and only a few are even sketched. One problem in writing this part was to find formulations of the results in terminology which would be understandable to both complex analysts and algebraic geometers, and which would at the same time combine the classical, geometric flavour of the subject while taking advantage of the generality and precision offered by the current homological formulations. I have chosen a compromise course, one which seemed best to me, and I have also parenthetically inserted alternate terminology and formulations when it seemed appropriate.

The Table of Contents for Part I is fairly self-explanatory, and I should only like to add a parenthetical remark about two main areas in algebraic geometry and function theory which are playing a central role in the study of period matrices. The first of these is Hironaka's resolution of singularities (cf. the end of $\$ 1$ ), which in practice permits us to have a suitable localization around singular points of algebraic varieties. The second of these is hyperbolic complex analysis (cf. §6) which, to paraphrase Chern, is the general philosophy that suitable curvature conditions on complex manifolds impose very strong restrictions on the holomorphic mappings between such manifolds.

A third area which should play an increasingly important role in variation of Hodge structures is group theory. Both the theory of algebraic groups and their arithmetic subgroups [10], and the theory of representations of real, semisimple Lie groups [24] have a close relation to many of the problems given in Part II. 
About $1 / 3$ of the results listed below have been announced in print, another $1 / 3$ exist in preprint form, and the remaining $1 / 3$ have yet to even reach that point. Except for the foundational results in \$2, essentially none have been published with proofs. Given this, I have still included references as much as was possible.

(ii) In Part II I have given a fairly complete discussion of those central problems and conjectures about period matrices of which I am aware. Several of these are conjectures of others (such as Andreotti-Weil's conjecture about $K-3$ surfaces- $\$ 7$, Brieskorn's problem about the Picard-Lefschetz transformation around isolated singular points of hypersurfaces- $\$ 8$, or Deligne's conjecture about the Hodge structure of a degenerating algebraic variety- $-\$ 9)$. Some of the problems (such as the $K-3$ conjecture) are long-standing, and I have simply written these down together with whatever recent information was available. Other questions are of recent origin, and I have tried to give a precise and thorough explanation of these.

I have also used a system of putting asterisks "*" alongside the problem or conjecture. A single asterisk means that the problem should be accessible to present techniques, and occasionally I have even given an outline of how the problem might be approached (e.g., (8.4) and (11.2)). Two asterisks means that the problem is one which is perhaps accessible by current methods, and which, at any event, seems to me as though it could be profitably worked on. Three asterisks means that the problem will most likely require completely new insight. Of course these "ratings" are my own opinions and nothing more.

(iii) In Part III I have given some examples pertaining to the problems and conjectures in Part II. The first of these ( $\$ 13)$ is essentially an old unpublished result of Mayer and Mumford which says basically that all of our general problems and conjectures are satisfactory for curves or abelian varieties.

In $\$ 14$ there is given an example, due jointly to Clemens and myself, of the periods of an algebraic surface which is degenerating in an interesting manner. (Contrary to the case of curves, the "general singularity" which a surface acquires is, from the point of view of Hodge structures, not particularly interesting.) This example gives nontrivial substantiation to some of the conjectures in $\$ 9$.

In $\$ 15$ I have discussed the monodromy theorem ( $\$ 3)$ while also giving some illustrations of the local invariant cycle problem (\$8).

The appendix, which was added to the galley proofs, discusses some recent progress on the problems and conjectures given in Part II.

In closing I should like to express my appreciation to the many 
colleagues with whom I have had helpful discussions and many of whose suggestions have been incorporated into this paper. In particular, Professor Grothendieck read over two preliminary versions of the manuscript and pointed out several statements which needed clarification.

\section{PART I. SUmmary OF MAIN RESUlts}

1. The geometric situation giving rise to variation of Hodge structure. All algebraic varieties will be defined over $\mathbf{C}$ and will be reduced. The ad ective smooth for an algebraic variety will mean nonsingular; thus a smooth algebraic variety $X$ may be considered as a complex manifold, and we will denote by $\theta_{X}$ (or simply $\theta$ if no confusion is possible) the sheaf of germs of holomorphic functions on $X$. A morphism $f: X \rightarrow Y$ between algebraic varieties will be a rational, holomorphic mapping. If $X$ and $Y$ are smooth, then a morphism $f: X \rightarrow Y$ will be smooth if the mapping between tangent bundles $f_{*}: T(X) \rightarrow T(Y)$ induced by the differential of $f$ has everywhere maximal rank.

The situation we will study is that of an algebraic family of algebraic varieties. To be precise, such a situation is given by a proper morphism $f: X \rightarrow S$ between algebraic varieties where we make the following assumptions:

(a) $X, S$, and $f$ are smooth;

(b) $X, S$, and the fibres $V_{s}=f^{-1}(s)(s \in S)$ of $f$ are connected; and

(c) we are given a distinguished class of projective embeddings $X \subset P_{N}$ (i.e., a polarization of $X$ ).

We may intuitively think of this situation as a nice family $\left\{V_{s}\right\}_{s \in S}$ of smooth, complete (i.e., compact), and projective algebraic varieties with an algebraic parameter space $S$. In general $S$ will not be complete, and we should have the picture that the $V_{s}$ are acquiring singularities as $s$ tends to infinity in $S$.

We shall also consider smooth compactifications of our situation $f: X \rightarrow S$. By this we shall mean a diagram

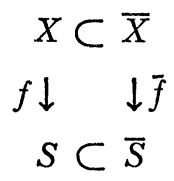

where $\bar{X}, \bar{S}$ are smooth and complete and where $\bar{X}-X$ and $\bar{S}-S$ are divisors with normal crossings. Thus, for example, $\bar{S}-S$ will be given locally by

$$
z_{1} \cdots z_{k}=0
$$


where $z_{1}, \cdots, z_{n}$ is a local holomorphic coordinate system on $\bar{S}$. The nonsingular divisors $z_{1}=0, \cdots, z_{k}=0$ will be referred to as the irreducible branches of $S$ at infinity. Another example is when $\operatorname{dim}_{C} S$ $=1$, so that $\bar{S}-S$ is given by $s=0$ for a local holomorphic coordinate $s$ on $\bar{S}$. Then $\bar{f}: \bar{X} \rightarrow \bar{S}$ will be given locally by

$$
x_{1}^{a_{1}} \cdots x_{r}^{a_{r}}=s
$$

where $x_{1}, \cdots, x_{r}$ is a local holomorphic coordinate system on $\bar{X}$.

Such smooth compactifications exist by the fundamental work of Hironaka. In fact, I should like to insert here the personal observation that Hironaka's work has found many important applications in the area of periods of integrals and Hodge structures. Among these applications are:

(i) Grothendieck's algebraic deRham theorem [5];

(ii) the local monodromy theorem stated in (3.1) below (cf. also $\S 15$ for the sketch of a proof);

(iii) the complete reducibility of global monodromy (3.3);

(iv) the regularity of the Picard-Fuchs equations (Theorem 4.1);

(v) the geometric analogue of Tate's conjecture (Theorem 5.1); and

(vi) the theorems applying hyperbolic complex analysis to the study of the behavior of period matrices at infinity (Theorems 6.3, 6.5 , and 6.6).

2. Data given by variation of Hodge structure. Given the situation $f: X \rightarrow S$ of $\S 1$, we may pass to the cohomology along the fibres and thereby effect a linearization of the geometric picture. Upon so doing the following data is obtained:

(a) A holomorphic vector bundle $\pi: E \rightarrow S$ with a canonical flat connexion $D$. Fixing a base point $s_{0} \in S$, we may define $E$ as being the holomorphic bundle, with constant transition functions, which is associated to the usual representation of the fundamental group $\pi_{1}\left(S, s_{0}\right)$ on the cohomology $H^{q}\left(V_{s_{0}}, C\right)$. The connexion $D$ is the usual flat connexion which one has on any bundle associated to a representation of the fundamental group.

The sheaf $\mathcal{O}(E)$ of holomorphic sections of $E$ contains the subsheaf $\mathcal{C}(E)=\{\phi \in \mathcal{O}(E): D \phi=0\}$ of locally constant sections of $E$. In fact, $\mathfrak{C}(E)$ is just the usual Leray sheaf $R_{f_{*}}^{q}(C)$, the latter, by definition, being the sheaf associated to the presheaf $U \rightarrow H^{a}\left(f^{-1}(U), \boldsymbol{C}\right)$ where $U$ runs through the family of open subsets of $S$. Following [5], $D$ is termed the Gauss-Manin connexion.

(b) A holomorphically varying filtration $F^{0} \subset \cdots \subset F^{q}=E$ (Hodge 
filtration) where the fibre $F_{s}^{r}$ of the holomorphic subbundle $F^{r}$ of $E$ is just $H^{q, 0}\left(V_{s}\right)+\cdots+H^{q-r, r}\left(V_{s}\right)$ viewed as a subspace of $H^{q}\left(V_{s}, C\right)$ $=E_{0}[2],[3]$.

(Grothendieck remarks that the above filtration of $E$ can be defined in a purely algebraic manner as follows: Let $\dot{\Omega}_{X / S}$ $=\bigoplus_{q=0}^{n=\operatorname{dim} V \cdot} \Omega_{X / S}^{q}$ be the algebraic coherent sheaf, in the Zariski topology, of relative differentials on $X$ which are simultaneously holomorphic and rational. The exterior differentiation on $\dot{\Omega}_{X}$ induces $d: \Omega_{X / S}^{q} \rightarrow \Omega_{X / S}^{a+1}$ which is $\Theta_{S}$-linear. Thus $\dot{\Omega}_{X / S}$ is a graded, differential sheaf and so the Leray hypercohomology sheaves $[5] R_{f_{*}}{ }^{*}\left(\dot{\Omega}_{X / S}\right)$ are defined and are coherent algebraic sheaves on $S$. By the main theorem in [5] we see that the analytic coherent sheaf associated to $R_{f_{*}}{ }^{*}\left(\dot{\Omega}_{X / S}\right)$ is just $R_{f_{*}}^{*}(\boldsymbol{C}) \otimes \Theta_{s}$. On the other hand there is the usual spectral sequence $\left\{E_{r}^{p, r}\right\}$ with $E_{1}^{p, r}=R_{f_{*}}^{r}(\Omega)_{X / S}^{p}$ and with $\bigoplus_{p+r=q} E_{\infty}^{p, r}$ being the graded sheaf associated to a filtration of $\left.\left\{\boldsymbol{R}_{f_{*}^{*}}{ }^{*} \dot{\Omega}_{X / S}\right)\right\}^{a}$ by coherent algebraic subsheaves. This is the algebraic definition of the filtration $F^{0} \subset \cdots \subset F^{q}=E$, since because of the degeneracy of the above spectral sequence the $F^{r}$ are just the holomorphic vector bundles associated to coherent algebraic subsheaves of $\left\{R_{f_{*}^{*}}^{*}\left(\dot{\Omega}_{X / S}\right)\right\}^{q}$.)

As conditions on the data (a) and (b) we have

(c) the Gauss-Manin connexion satisfies

$$
D: \Theta_{S}\left(F^{p}\right) \rightarrow \Omega_{S}^{1}\left(F^{p+1}\right) .
$$

This is the infinitesimal period relation or transversality condition [2]. Passing to the associated graded situation in (2.1), we see that $D$ induces an $\hat{\theta}_{S}$-linear mapping

$$
\widetilde{D}: \Theta_{S}\left(F^{p} / F^{p-1}\right) \rightarrow \Omega_{S}^{1}\left(F^{p+1} / F^{p}\right) .
$$

If we now denote by $\Omega_{X / S}^{r}$ the sheaf on $X$ of relative, holomorphic $r$-forms, then $\Theta_{S}\left(F^{p} / F^{p-1}\right)$ is just the Leray sheaf $R_{f}^{p}\left(\Omega_{X / S}^{q-p}\right)$. Thus, in ( $\widetilde{2.1}$ ) we see that $\widetilde{D}$ is given by a global section $\widetilde{D}$ of

$$
\operatorname{Hom}_{\Theta_{S}}\left(R_{f_{*}^{p}}^{p}\left(\Omega_{X / S}^{q-p}\right), R_{f_{*}}^{p+1}\left(\Omega_{X / S}^{q-p-1}\right)\right) \Omega \otimes_{S}^{1} .
$$

From [2] it follows that this $\tilde{D}$ is just the cup product with the Kodaira-Spencer infinitesimal deformation class

$$
\theta \in R_{f_{*}}^{1}\left(\operatorname{Hom}\left(\Omega_{X / S}^{1}, \mathcal{O}_{X}\right)\right) \otimes \Omega_{S}^{1} .
$$

(d) On each fibre $F_{s}^{0} \subset F_{s}^{1} \subset \cdots \subset F_{s}^{q-1} \subset E_{s}$ of the situation (b), there is defined the Hodge bilinear form $Q$, which is a bilinear form, flat with respect to $D$, and with the properties given in Hodge [6] or Weil [9]. 
We now want to formulate the data and conditions (a)-(d) just given in an equivalent, but perhaps more geometric, manner. The idea is to use a classifying space for Hodge filtrations. More specifically, we reconsider $E$ as now being the bundle formed from the primitive cohomology $H^{q}\left(V_{\varepsilon_{0}}, C\right)_{0}$ [2], [9]. Recall that the rational primitive cohomology $H^{q}\left(V_{s_{0}}, \boldsymbol{Q}\right)_{0}$ is, by definition, the kernel of

$$
L^{n-q+1}: H^{q}\left(V_{s_{0}}, Q\right) \rightarrow H^{2 n-q+2}\left(V_{s_{0}}, Q\right)
$$

where $L \in H^{2}\left(V_{s_{0}}, Q\right)$ is the cohomology class of a hyperplane section of $V_{s_{0}}$ relative to the given embedding $X \subset P_{N}$. It is clearly the case that $L$ operates on the Leray sheaves $R_{f}^{q}(Q)$, so that $\pi_{1}\left(S, s_{0}\right)$ leaves $H^{q}\left(V_{s_{0}}, Q\right)_{0}$ invariant as a subspace of ${ }^{*} H^{q}\left(V_{s_{0}}, Q\right)$. Because of the Lefschetz decomposition

$$
H^{q}\left(V_{s_{0}}, Q\right)=\sum_{r} L^{r} H^{q-2 r}\left(V_{s_{0}}, Q\right)_{0},
$$

any information about $H^{q}\left(V_{s_{0}}, Q\right)$ is a direct sum of information about the primitive cohomology groups. The reason for passing to the primitive cohomology is that the Hodge bilinear form $Q$ now has the nice positive-definite sign properties given in [6] and recalled in (9.8), (9.9) below.

Then we construct, as in [2], [3], the period matrix space $D$ of all possible inequivalent Hodge structures on a given vector space $E$ $\left(=H^{q}\left(V_{s_{0}}, \boldsymbol{C}\right)_{0}\right)$ having fixed $\boldsymbol{Z}$ lattice (=image of $\left.H^{q}\left(V_{s_{0}}, \boldsymbol{Z}\right)_{0}\right)$ and rational bilinear form $Q$ (= Hodge bilinear form on $\left.H^{q}\left(V_{s_{0}}, C\right)_{0}\right)$, and having given Hodge numbers $h^{a, b}\left(=\operatorname{dim} H^{a, b}\left(V_{s_{0}}\right)_{0}\right)$. The data and conditions (a), (b), (d) above are equivalent to giving a holomorphic mapping (period mapping)

$$
\Phi: S \rightarrow D / \Gamma
$$

where $\Gamma \subset \operatorname{Aut}_{z}(E)$ is the global monodromy group (which, by definition, is the image of $\pi_{1}\left(S, s_{0}\right)$ in $\left.\operatorname{Aut}_{z}\left\{H^{q}\left(V_{s_{0}}, C\right)_{0}\right\}\right)$.

To explain the infinitesmial period relation (2.1) in (c), we recall from [2] and [4] that $D$ is in a natural way a homogeneous complex manifold $H \backslash G$, where $G$ is the identity component of the real orthogonal group of the bilinear form $Q$, and where $H$ is the compact subgroup of $G$ which leaves a given Hodge structure invariant. Letting $K$ be the maximal compact subgroup of $G$ and $R=K \backslash G$ the corresponding Riemannian symmetric space, in the equivariant fibering

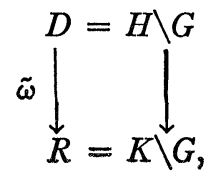


there is a unique, $G$-invariant connexion

$$
T_{\Omega}(D)=V_{\Omega} \oplus H_{\Omega}
$$

$(\Omega \in D)$ where the vertical space $V_{\Omega}$ is tangent to the $K$-orbit of $\Omega \cdot K$ in $D$ and $H_{\Omega}$ is the complementary horizontal space [4]. Now the condition (c) above implies to the statement:

(2.4). The period mapping $\Phi: S \rightarrow D / \Gamma$ is horizontal in the sense that $\Phi_{*}(t) \in H_{\Phi(s)}$ for a tangent vector $t \in T_{s}(S)$.

In general, a locally liftable holomorphic mapping $\Psi$ from a complex variety $W$ to $D / \Gamma$ is said to be horizontal if the condition on the differential $\Psi_{*}$ given in (2.4) is true at simple points of $W$. (Recall that a holomorphic mapping $\Psi: W \rightarrow D / \Gamma$ is locally liftable if, for each point $w \in W$ there is a neighborhood $U_{w}$ of $w$ in $W$ and a holomorphic mapping $\tilde{\Psi}: U_{w} \rightarrow D$ such that the diagram

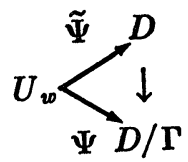

commutes.)

It is perhaps worth recalling here that the fibering (2.3) has the following properties [2]:

(i) the projection is not holomorphic unless $H=K$ and $D$ is a bounded symmetric domain in the sense of $\mathrm{E}$. Cartan;

(ii) the fibres $\tilde{\omega}^{-1}(r)$ are all nonsingular, complex submanifolds of $D$ which are biregularly equivalent to the rational, homogeneous projective variety $H \backslash K$; and

(iii) $R$ may roughly be thought of as the set of "real points" in the "Chow variety of all deformations of a fixed fibre $\tilde{\omega}^{-1}\left(r_{0}\right)$ in $D$ " (cf. Remark 11. 2 below).

3. Theorems about monodromy of homology. There are two main results here: the essential unipotence of the local action of $\pi_{1}(S)$ on $H^{q}\left(V_{s}, C\right)$ around the branches of $\bar{S}-S$ at infinity (this is the so-called monodromy theorem), and the global complete reducibility of the monodromy group $\Gamma$.

(a) The local theorem is this: We localize $\bar{f}: \bar{X} \rightarrow \bar{S}$ around a single irreducible branch of $\bar{S}-S$ and let $T: H^{q}\left(V_{s}, \boldsymbol{C}\right) \rightarrow H^{q}\left(V_{s}, \boldsymbol{C}\right)$ be the Picard-Lefschetz transformation (P.-L. transformation) obtained by transporting cycles around the branch of $\bar{S}-S$ [3], [7], [8], [19]. A more precise description of this localization is given in $\$ 8$ below.

(3.1) Theorem (Monodromy theorem). (i) The eigenvalues of $T$ are roots of unity, so that $T^{N}$ is unipotent for some $N>0$. 
(ii) The elementary divisors of $T^{N}$ are less than $q+1$, so that $\left(T^{N}-I\right)^{q+1}=0$.

(3.2). REMARK. There are four methods of proof of this theorem which I know of, and which I will discuss briefly.

(P-1). The first is the original topological argument based on the methods of Lefschetz (Landman [22]) and the direct topological proof by Clemens [14]. Grothendieck has also found a cohomological proof which is similar to Clemens'. In $\$ 15$ we will sketch this proof in conjunction with a discussion of one of our problems. Both arguments use the resolution of singularities of a singular fibre of $\bar{f}: \bar{X} \rightarrow \bar{S}$, and each gives estimates on $N$ and on the index of nilpotency of $T^{N}-I$.

(P-2). The second is Grothendieck's original arithmetic argument [19], which also uses resolution of singularities.

(P-3). The third is a combination arithmetical and analytic argument based on the fact that the Picard-Fuchs equations (GaussManin connexion) have regular singular points ([3] and $\$ 4$ below). As best as I can determine the two proofs of this kind are due to Brieskorn and Grothendieck-Katz. These methods do not require resolution of singularities, but the proofs I have seen only give the eigenvalue statement (i) in Theorem 3.1. An outline of this proof is given following Theorem 4.1 below.

(P-4). The last is a proof using hyperbolic complex analysis (cf. $\S 6$, and (8.3), (8.4) below), this argument being due to Borel. Here again use is not made of resolution of singularities, and thus far Borel gets out only the eigenvalue statement-cf. Problem (8.3) in $\S 8$ below.

(b) The main global theorem is the following result of Deligne [1] (cf. Lefschetz [7] and Hodge [6] for special cases).

(3.3) Theorem. The global monodromy group $\Gamma \subset \operatorname{Aut}_{Q}\left(H^{q}\left(V_{s_{0}}, C\right)\right)$ is completely reducible.

(3.4). REMARK. In outline, Deligne's proof of semisimplicity goes as follows: First, Deligne observes that the Leray spectral sequence of $f: X \rightarrow S$ degenerates at the $E_{2}$-term, so that in particular we have

$$
H^{*}(X, C) \stackrel{r}{\rightarrow} H^{0}\left(S, R_{f}^{*}(C)\right) \rightarrow 0 .
$$

Deligne then proves the basic fact that, in the diagram

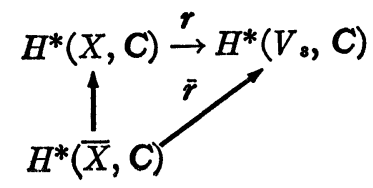


the image of $r$ equals that of $\bar{r}$. This proof is based on his notion of "weight filtration and generalized Hodge structure" on $H^{*}(X, \boldsymbol{C})$ (cf. $\$ 9$ below). Consider now the invariant part $I^{*}=H^{*}\left(V_{s_{0}}, \boldsymbol{C}\right)^{\pi_{1}(\boldsymbol{S})}$ $=H^{0}\left(S, R_{f}^{*}(\boldsymbol{C})\right)$. According to (3.5) and (3.6), $I^{*}$ is the image in $\bar{r}: H^{*}(\bar{X}, \boldsymbol{C}) \rightarrow H^{*}\left(V_{s_{0}}, \boldsymbol{C}\right)$ and is thus a "sub-Hodge-structure"; in particular, using the Hodge inner product, we have a $\pi_{1}(S)$-invariant splitting $H^{*}\left(V_{\boldsymbol{s}_{0}}, \boldsymbol{C}\right)=I^{*} \oplus\left(I^{*}\right)^{\perp}$. If now $F \subset H^{q}\left(V_{\boldsymbol{s}_{0}}, \boldsymbol{C}\right)$ is a maximal $\pi_{1}(S)$-invariant subspace, then, if $\operatorname{dim} F=l$, the $l$ th exterior power $\Lambda^{l} F \subset \Lambda^{l}\left\{H^{q}\left(V_{s_{0}}, C\right)\right\}$ gives an invariant line in

$$
\begin{gathered}
H^{q l}\left(V_{s_{0}} \times \cdots \times V_{s_{0}}, C\right), \\
(l \text { times })
\end{gathered}
$$

and we can find an invariant vector generating this line and proceed as before.

4. Theorems about Picard-Fuchs equations (Gauss-Manin connexion). The basic general fact is the result announced in [3] that the Picard-Fuchs equations have regular singular points. This is essentially equivalent to saying that the "periods" in our family $f: X \rightarrow S$ do not have essential singularities on $\bar{S}-S$, or what amounts to the same thing, that these periods have polynomial growth at infinity. Before stating the theorem, we will define the notion of regular singular points in our context.

Let $S$ be a smooth algebraic variety and $E \rightarrow S$ an algebraic vector bundle with a flat algebraic connexion $D$. (This applied to our situation as was remarked in (b) of $\$ 2$ ). We will say that $D$ has regular singular points at infinity on $S$ if the following is true:

For any smooth compactification $\bar{S}$ of $S$ given locally by (1.1), given $\bar{s} \in \bar{S}-S$ there is a topological neighborhood $U$ of $\bar{s}$ in $\bar{S}$ and rational sections $\phi_{1}, \cdots, \phi_{m}$ of $E \rightarrow S$ with the following properties:

(i) $\phi_{1}, \cdots, \phi_{m}$ are holomorphic in $U \cap S$ and give a basis for all of the fibres $E_{s}(s \in U \cap S)$; and

(ii) the connexion $D$ is given by

$$
D \phi_{j}=\sum_{\alpha=1}^{k} A_{j \alpha}^{i} \phi_{i} \otimes \frac{d z_{\alpha}}{z_{\alpha}}+\sum_{\mu=k+1}^{n} A_{j \mu}^{i} \phi_{i} \otimes d z_{\mu}
$$

where the $A_{j \alpha}^{i}, A_{j \mu}^{i}$ are holomorphic in $U$. Thus, e.g., if $\operatorname{dim} S=1$, then $D$ will have a regular singular point at $s=0$ if

$$
D \phi_{j}(s)=\sum_{i=1}^{m} A_{j}^{i}(s) \phi_{i}(s) \otimes \frac{d s}{s} .
$$


(4.3). Theorem (Regularity theorem). The Gauss-Manin connexion has regular singular points. Moreover, there exists a locally free coherent analytic sheaf $\Theta_{\bar{S}}(E)$ on $\bar{S}$ such that:

(i) $\mathcal{O}_{\bar{S}}(E)$ restricted to $S$ is just $\mathcal{O}_{S}(E)$; and

(ii) if $\phi$ is a local section of $\mathcal{O}_{\bar{S}}(E)$ in a neighborhood of $\bar{s} \in S-\bar{S}$, then

$$
D \phi=\sum_{j=1}^{k} \phi_{j} \otimes \frac{d z_{j}}{z_{j}}+\sum_{l=k+1}^{n} \phi_{l} \otimes d z_{l}
$$

where $\phi_{1}, \cdots, \phi_{n}$ are sections of $\mathcal{O}_{\bar{S}}(E)$.

REMARKs. We shall outline the original proof of (4.1) and then comment on two very interesting recent proofs by Deligne and Katz. Consider for simplicity the case $\operatorname{dim} S=1$ and let $s$ be a local holomorphic coordinate on $\bar{S}$ such that $\bar{S}-S$ is given by $s=0$. Fix $s_{0}$ near to $s$ and let $\Psi_{1}, \cdots, \Psi_{m}$ be a basis for $H^{q}\left(V_{s_{0}}, C\right)$. Then $\Psi_{1}, \cdots, \Psi_{m}$ will displace to a many-valued basis for $H^{q}\left(V_{s}, \boldsymbol{C}\right)$ in the punctured disc $0<|s|<\epsilon$. The indeterminacy in this procedure is given exactly by the P.-L. transformation $T$ discussed in $\S 3$.

For simplicity we assume that the eigenvalues of $T$ are all equal to 1. The case when all eigenvalues are equal to a fixed number $\rho$ is done exactly the same way, and the general case is then done by writing $H^{q}\left(V_{s_{0}}, C\right)$ as a direct sum of subspaces on which $T$ has a single eigenvalue. If we define

$$
\log T=(T-I)-(T-I)^{2} / 2+\cdots+(-1)^{q-1}(T-I)^{q} / q,
$$

then the matrix $\log T$ has the properties:

(i) $(\log T)^{a+1}=0$, and

(ii) $T=e^{\log T}=I+\log T+\cdots+\log T / q^{1}$ is a polynomial in $\log T$.

Let now $\Phi_{1}, \cdots, \Phi_{m}$ be any rational sections of $E \rightarrow S$ which are holomorphic and give a basis of $E_{s}$ for $0<|s|<\epsilon$. Then we will have

$$
\Phi_{j}(s)=\sum_{i=1}^{m} C_{j i}(s) \Psi_{i}
$$

where the matrix $C(s)=\left(C_{j i}(s)\right)$ is locally holomorphic in $0<|s|<\epsilon$ and satisfies

$$
C\left(e^{2 \pi i} s\right)=C(s) \cdot T,
$$

where $C\left(e^{2 \pi i} s\right)$ denotes the result of analytically continuing $C(s)$ around $s=0$. The entries $C_{j i}(s)$ are to be thought of as integrals

$$
\int_{\gamma} \Phi_{j}(s)
$$


of rational differential forms $\Phi_{j}(s)$ on $V_{s}$ which depend rationally on $s \in S$, and where the $\gamma_{i}$ are cycles in $H_{q}\left(V_{s}, Q\right)$. The main step in the proof of (4.1) is to prove the estimate

$$
\left|\int_{\gamma_{\boldsymbol{i}}} \Phi_{j}(s)\right| \leqq a|s|^{-b}|\log s|^{\bullet}
$$

for suitable constants $a, b, c$. This estimate is done using the method of Lefschetz [7] to describe the cycles on $V_{s}$ as $s$ tends to zero.

Now let $l(s)=\log s / 2 \pi i$ and write

$$
C(s)=B(s) e^{[l(s) \cdot \log T]} .
$$

By (4.4) we have $B\left(e^{2 \pi i} s\right)=B(s)$, and it follows from (4.5) and the fact that $e^{[l(s) \log T 1}=\sum_{\mu=0}^{q} l(s)^{\mu}(\log T)^{\mu}$ is a polynomial in $\log (s)$ that $B(s)=\sum_{\nu=-N}^{\infty} B_{\nu} s^{\nu}$ is single-valued and meromorphic at $s=0$, and holomorphic with $\operatorname{det} B(s) \neq 0$ in $0<|s|<\epsilon$. We may then obviously assume that our rational sections $\Phi_{1}, \cdots, \Phi_{m}$ have been selected so as to give a holomorphic basis for $E_{s}$ in $0<|s|<\epsilon$ and such that the matrix $B(s)$ in (4.6) is holomorphic in the whole disc $0 \leqq|s|<\epsilon$ and $\operatorname{det} B(0) \neq 0$. Since $D \Psi_{i}=0$ it follows that $D \Phi_{i}=\sum_{j} \theta_{j i} \Phi_{j}$ where

$$
\theta=d B \cdot B^{-1}+\left(B \log T B^{-1}\right) d s / s
$$

is the connexion matrix. Comparing this with (4.2) we find our theorem.

The proof by Deligne is of a somewhat more general result and is also based on the characterization of a flat, algebraic connexion with regular singular points by growth estimates similar to (4.5).

Before discussing Katz' argument, we remark (as was done in [3]) that (4.3) is a purely algebraic theorem whose proof was, however, based on analysis. This state of affairs has just been remedied and clarified by Katz, who gives a purely algebraic proof of the regularity theorem and which I will now outline.

(i) The first step is that the Gauss-Manin connexion admits a purely algebraic definition (cf. Katz and Oda, J. Math. Kyoto Univ. 8 (1968), 31-45). This is an extension of Grothendieck's comment given in $\S 2(\mathrm{~b})$. Thus we may think of $D$ as a rational differential operator

$$
D: R_{f_{*}}^{*}\left(\dot{\Omega}_{X / S}\right) \rightarrow R_{f_{*}}^{*}\left(\dot{\Omega}_{X / S}\right) \otimes \Omega_{S}^{1}
$$

where $R_{f_{*}}^{*}\left(\Omega_{X / S}\right)$ is the algebraic coherent sheaf coming from the presheaf $U \rightarrow H^{*}\left(f^{-1}(U), \dot{\Omega}_{X / S}\right)$ where $U$ is a Zariski open set in $S, \dot{\Omega}_{X / S}$ is 
the algebraic coherent sheaf of relative rational, holomorphic differentials on $X$, and $H^{*}\left(f^{-1}(U), \dot{\Omega}_{X / S}\right)$ means hypercohomology.

(ii) From (i) it follows that $D$ may be "reduced modulo $p$ " for almost all primes $p$ whenever the same is done to the situation $f: X \rightarrow S$. Katz then observes that, in the reduced situation modulo $p$ for almost all $p$, we have

$$
D^{M}=0
$$

for a suitable $M=M(p)$, this being related to the fact that, for rational, holomorphic functions $f(x)$, we have $d^{k} f(x) / d x^{k} \equiv 0$ (modulo $p$ ) for $k \geqq p$. We may think of (4.7) as the nilpotence of the Gauss-Manin connexion in characteristic $p$.

(iii) From the nilpotency of the Gauss-Manin connexion in characteristic $p$ for almost all $p$, Katz uses the following result of Turrittin (Acta Math. 93 (1955), 27-66) to deduce the regularity theorem: Let $A(z)=\sum_{\mu=-m}^{\infty} A_{\mu} z^{\mu}$ be a meromorphic (in the usual sense) $n \times n$ matrix defined on the disc $|z|<1$ which is holomorphic for $0<|z|<1$, and consider the system of ordinary differential equations

$$
d u(z) / d z=A(z) u(z)
$$

for an unknown vector function $u(z)$ in $0<|z|<1$. Then, by a change of variable $z=t^{k}$ and change of coordinates $B(t)=C(t) A(t)$ where $C(t)=\sum_{\nu=-l}^{\infty} C_{\nu} t^{\nu}$ is meromorphic in $|t|<1$ and holomorphic with $\operatorname{det} C(t) \neq 0$ in $0<|t|<1$, we may bring the system (4.8) into the canonical form

$$
d v(t) / d t=B(t) v(t)
$$

where $B(t)=\sum_{\mu=-h}^{\infty} B_{\mu} t^{\mu}$ and where $B_{-h}$ is not nilpotent if $h>1$.

The link between the nilpotency of the Gauss-Manin connexion in characteristic $p$ and (4.9) is roughly that, if we set $D=\{d / d t-B(t)\}$, then we cannot have $D^{M} \equiv 0$ (modulo $p$ ) for infinitely many primes $p$ if $h>1$ because $B_{-h}$ is not nilpotent in (4.9). Thus we must have $h=1$ and so (4.9) has a regular singular point at $t=0$.

The proof of the eigenvalue statement in the monodromy Theorem 3.1 also follows by a similar argument, since in fact $T=\exp \left(B_{-1}\right)$ in (4.9) while $B_{-1}$ must be nilpotent for the same reasons as before.

There are three applications which I know of the regularity theorem.

(a) The first is in the proof of the monodromy theorem discussed in Remark 3.4 and given in outline form just above. 
(b) The next is Deligne's generalization [15] of Grothendieck's algebraic deRham theorem [5]. For affine $S$, this result is:

(4.10). THEOREM. The cohomology $H^{*}\left(S, R_{*}^{*}(C)\right)$ is the same as the deRham cohomology of the complex of rational, holomorphic differential forms with values in $E$ (this has a meaning because of Theorem 4.3).

(c) The last application of Theorem 4.3 is the first, and fairly crude, result on the behavior of the period mapping (2.2) at infinity (or what amounts to the same thing, the limiting position of the Hodge filtration of $H^{q}\left(V_{s}, C\right)$ as s tends to $\left.\bar{s} \in \bar{S}-S\right)$. To explain this result, I first recall that there is naturally associated to $D$ a dual $\check{D}$ with the following properties [4]:

(i) $\check{D}$ is a rational, homogeneous projective algebraic variety of the form $\check{D}=B \backslash \breve{G}$ where $\check{G}$ is a complex, simple Lie group and $B$ is a parabolic subgroup;

(ii) the period matrix domain $D=H \backslash G$ where $G$ is a real form of $\check{G}$ and $H=G \cap B$; and

(iii) from (ii) it follows that there is a natural equivariant embedding $D \subset \check{D}$ whose topological boundary $\partial D=\bar{D}-D$ is a disjoint union of $G$-orbits.

The relation of $\check{D}$ to Hodge structures is the following (cf. [2], [3]): If we think of $D$ as all points on a flag manifold which satisfy the two Hodge bilinear relations $Q(\Omega, \Omega)=0$ and $Q(\Omega, \bar{\Omega})>0$, then $\check{D}$ is just these $\Omega$ which satisfy the first relation $Q(\Omega, \Omega)=0$ (cf. $\$ 13$ below where the case $q=1$ (Siegel-upper-half-plane) is discussed in this setting).

We now localize the period mapping around an irreducible branch $B$ of $\bar{S}-S$. If $z^{1}, \cdots, z^{n}$ is a coordinate system on $\bar{S}$ such that $B$ is given by $z^{1}=0$, we set $z^{1}=\exp (w), z=\left(z^{2}, \cdots, z^{n}\right)$, and lift the period mapping to

$$
\begin{array}{r}
H \times \Delta^{n-1}=H \times{ }^{-} \Delta \times \cdots \times \Delta \\
(n-1 \text { times })
\end{array}
$$

where $H$ is the usual upper-half-plane $\{w: \operatorname{Im} w>0\}$ and $\Delta$ is an ordinary disc in $C$. The lifted map $\Phi: H \times \Delta^{n-1} \rightarrow D$ satisfies

$$
\Phi(w+1, z)=\Phi(w, z) \cdot T
$$

where $T: H^{q}\left(V_{s}, \mathbf{C}\right) \rightarrow H^{q}\left(V_{s}, \mathbf{C}\right)$ is the P.-L. transformation obtained by turning around $B$.

(4.11). Theorem. The limit $\lim _{\operatorname{Im} w \rightarrow \infty} \Phi(w, z)$ exists as a point $\Phi(0, z) \in \bar{D}$ and has the properties: 
(i) $\Phi(0, z)=\Phi(0, z) \cdot T$ is a fixed point of $T$, and

(ii) $\Phi(0, z)$ depends holomorphically on $z=\left(z^{2}, \cdots, z^{n}\right)$.

REMARK. In particular if $T$ is of infinite order, then $\Phi(0, z) \in \partial D$ $=\bar{D}-D$ (cf. (6.4) below for the complement to this remark). Examples of Theorem 4.11 are given by (13.28) and (14.38) below. The general problem of describing the limiting period matrix in a family of degenerating algebraic varieties is discussed in $\$ 9$.

5. Global theorems about holomorphic and locally constant cohomology classes. Let $S$ be a smooth algebraic variety and $E \rightarrow S$ a holomorphic vector bundle with a flat holomorphic connexion

$$
D: \mathcal{O}_{S}(E) \rightarrow \Omega_{S}^{1}(E)
$$

as was discussed in $\S 2$. We suppose that $E$ has a flat conjugation $e \rightarrow \bar{e}$, a flat bilinear form

$$
Q: E \otimes E \rightarrow C, \quad Q\left(e, e^{\prime}\right)=(-1)^{q} Q\left(e^{\prime}, e\right),
$$

and a holomorphic filtration $F^{0} \subset F^{1} \subset \cdots \subset F^{q-1} \subset F^{q}=E$ such that the conditions (c) and (d) of $\$ 2$ are satisfied. We call $\varepsilon=(E, D, Q$, $\left.\left\{F^{k}\right\}\right)$ a variation of Hodge structure, and we observe that it is not necessary that $\mathcal{E}$ arise from an algebraic family of algebraic varieties $f: X \rightarrow S$. In case $\varepsilon$ does come from linearizing such a family as discussed in $\$ 2$, we shall say that $\varepsilon$ arises from a geometric situation. For the obvious reasons it is desirable to prove as much as possible about a variation of Hodge structure $\varepsilon=\left(E, D, Q,\left\{F^{k}\right\}\right)$ without assuming that it arises from a geometric situation.

We shall give some results about a variation of Hodge structure $\&$ when the base space $S$ is complete. These were given in [16] where it was falsely claimed that the results held for general $S$ (this claim was based on the assertion that Conjecture 8.1 below was "obvious"). In [1] Deligne has given an independent treatment of some of the material below and, using (3.5) and (3.6), he is able to remove the assumption that $S$ is complete in case $\varepsilon$ arises from a geometric situation $f: X \rightarrow S$.

Our methods are based on the structure equations of the variation of Hodge structure $\varepsilon=\left(E, D, Q,\left\{F^{k}\right\}\right)$, especially the curvature properties of the Hodge bundles $E^{k}=F^{k} / F^{k-1}$, together with use of the maximum principle. These structure equations, especially those arising from the infinitesimal period relation (2.1), are quite interesting in themselves.

The first result is 
(5.1) Theorem. Assume that $S$ is complete and that $\phi$ is a global holomorphic section of $F^{k}$ such that $D \phi$ projects to zero in $E / F^{k}$. Then there exists a global, constant section $\psi$ of $E$ such that $\phi-\psi$ is a section of $F^{k-1}$.

(5.2). Corollary. If $\phi$ is a section in $H^{0}(S, \mathfrak{e}(E))$, then the Hodge $(p, q)$ components of $\phi$ are also global sections of $\mathfrak{C}(E)$.

This corollary is given in [1] in case $\varepsilon$ arises from a geometric situation $f: X \rightarrow S$ where $S$ is arbitrary. As mentioned above, the proof of (5.1) follows from the maximum principle and a formula for the curvature in $E^{k}$ which we shall now explain. Recall first that if $H \rightarrow S$ is an arbitrary holomorphic vector bundle which has a (not necessarily positive-definite) Hermitian metric, then there is a unique metric connexion

$$
D_{H}: A^{0}(H) \rightarrow A^{1}(H) \quad\left(A^{q}(H)=C^{\infty} H \text {-valued } q \text {-forms on } S\right)
$$

which satisfies

(i) the metric in $H$ is flat, and

(ii) $D_{H}^{\prime \prime}=\bar{\partial}$ where $D_{H}=D_{H}^{\prime}+D_{H}^{\prime \prime}$ is the type decomposition of $D_{H}$. For example, if we take the Hermitian metric

$$
\left(e, e^{\prime}\right)=(-1)^{q} Q\left(e, \bar{e}^{\prime}\right)
$$

in $E$, then the metric connexion $D_{E}$ is just $D$. The Hodge bilinear form also induces Hermitian metrics in $F^{k}$ and $E^{k}$, where the metric in the latter bundle is positive definite. Returning to the general situation $H \rightarrow S$, the curvature $\Theta_{H}$ of the metric connexion is a $\operatorname{Hom}(H, H)$ valued $(1,1)$ form defined by

$$
\left(D_{H}\right)^{2} \psi=\Theta_{H} \cdot \psi \quad\left(\psi \in A^{0}(H)\right) .
$$

Thus, for example, $\Theta_{E}=0$. To give the formula $\Theta_{E}{ }^{k}$ for the metric curvature in the Hodge bundles, we refer to $(\tilde{2.1})$ and let

$$
\sigma_{k}: E^{k} \rightarrow E^{k+1} \otimes T^{*}
$$

( $T^{*}=$ cotangent bundle of $S$ ) be the linear bundle maps induced by $D$. Using the (positive-definite) Hermitian structure in the $\left\{E^{k}\right\}$, we may interpret

$$
\left(\sigma_{k}, \sigma_{k}\right)-\left({ }^{t} \sigma_{k-1},{ }^{t} \sigma_{k-1}\right) \quad\left(\sigma_{-1}=0=\sigma_{q+1}\right)
$$

as a $\operatorname{Hom}\left(E^{k}, E^{k}\right)$-valued $(1,1)$ form on $S$, which has a local expression $A \wedge t \bar{A}-B \wedge^{t} \bar{B}$ where $A, B$ are matrices of $(1,0)$ forms on $S$.

(5.3). Theorem. $\Theta_{E}{ }^{k}=\left(\sigma_{k}, \sigma_{k}\right)-\left({ }^{t} \sigma_{k-1},{ }^{t} \sigma_{k-1}\right)$. 
We have two applications of Theorem 5.1. To explain the first we let $\varepsilon=\left(E, D, Q,\left\{F^{k}\right\}\right)$ be a variation of Hodge structure and observe that $\varepsilon$ gives rise to a period matrix domain $D$, global monodromy group $\Gamma$, and period mapping $\Phi: S \rightarrow D / \Gamma$.

(5.4). Theorem (Rigidity theorem). Assume that $\mathcal{E}, \mathcal{E}^{\prime}$ are two variations of Hodge structure giving rise to period mappings $\Phi, \Phi^{\prime}$. Assume furthermore that

(i) either $S$ is complete or $\mathcal{E}$ and $\mathcal{E}^{\prime}$ both arise from geometric situations;

(ii) there is an isomorphism $\Psi: E_{8_{0}} \rightarrow E_{s_{0}}$ which is a $\Gamma$-invariant isomorphism of polarized Hodge structures.

Then the period mappings $\Phi$ and $\Phi^{\prime}$ coincide.

REMARKS. This rigidity theorem is due to Grothendieck [18] when $q=1$; it was also Grothendieck who conjectured (5.2) on the basis of the Tate conjectures. In case the period matrix domain $D$ is a bounded symmetric domain, a function-theoretic proof of (5.4) has been given by Borel-R. Narasimhan [11].

-The second application is a Mordell-Weil theorem for families of intermediate Jacobians. Let $f: X \rightarrow S$ be an algebraic family of algebraic varieties as in $\$ 1$ and let $V_{s}=f^{-1}(s)$. We set

$$
H_{+}^{2 q+1}\left(V_{s}\right)=H^{2 q+1,0}\left(V_{s}\right)+\cdots+H^{q+1, q}\left(V_{s}\right)
$$

and let

$$
T\left(V_{s}\right)=H_{+}^{2 q+1}\left(V_{s}\right) \backslash H^{2 q+1}\left(V_{s}, C\right) / H^{2 q+1}\left(V_{s}, Z\right)
$$

be the qth intermediate Jacobian of $V_{\text {s }}$ (cf. [2], [3], [23]). For $q=0$, $T\left(V_{s}\right)$ is the Picard-variety of $V_{s}$, and for $q=n-1 \quad\left(n=\operatorname{dim}_{C} V_{s}\right)$, $T\left(V_{s}\right)$ is the Albanese-variety of $V_{s}$.

(5.6). Proposition. There is a complex manifold $\mathrm{J}$ which is an analytic fibre space $\tilde{\omega}: J \rightarrow S$ of complex tori with $\tilde{\omega}^{-1}(s)=T\left(V_{s}\right)$ for $s \in S$.

Let $\mathcal{O}_{S}(\mathfrak{J})$ be the sheaf of holomorphic cross-sections of $\tilde{\omega}: \mathfrak{J} \rightarrow S$. From (2.1) and the definition of $T\left(V_{s}\right)$ we have an induced sheaf homomorphism

$$
D_{\jmath}: \Theta_{S}(J) \rightarrow \Omega_{S}^{1}\left(E / F^{q+1}\right)
$$

To explain the geometric meaning of $D_{\zeta}$, we let $\Theta\left(V_{s}\right)$ be the group of algebraic $(n-q-1)$-cycles on $V$, which are homologous to zero. Then 
there is an Abel-Jacobi homomorphism [3 ], [23 ]

$$
\phi: \Theta\left(V_{s}\right) \rightarrow T\left(V_{s}\right),
$$

which for $q=0$ and $n-1$ is just the classical mapping of divisors and zero-cycles on $V_{s}$ to the respective Picard and Albanese varieties. Let now $\Theta(S)$ be the sheaf on $S$ which assigns to each open set $U \subset S$ the group $\Theta(U)$ of analytic cycles $Z$ in $f^{-1}(U)$ which are of codimension $q+1$ on $f^{-1}(U)$ and have the properties that all intersections $Z_{s}=Z \cdot V$. are proper and that $Z_{s}$ is an algebraic $(n-q-1)$-cycle on $V_{s}$ which is homologous to zero. Then the Abel-Jacobi maps (5.8) fit together to give an Abel-Jacobi mapping

$$
\nu: \Theta(S) \rightarrow \mathcal{O}_{S}(\mathcal{J})
$$

depending holomorphically on parameters.

(5.10). Proposition. The homomorphism $\nu$ in (5.9) satisfies $D_{\zeta}(\nu)$ $=0$ in (5.7).

Our main result is

(5.11). TheOREM (MORdELL-WEIL FOR FAMILIES OF INTERMEdiate Jacobians). Assume $S$ is complete. Then the group $\operatorname{Hom}(S, J)$ of holomorphic sections $\nu$ of $J \rightarrow S$ which satisfy $D_{\mathfrak{J}} \nu=0$ is an extension of the intermediate Jacobian $T(X)$ of $X$ (the trace or fixed part) by a finitely generated abelian group.

REMARK. This theorem should remain true for arbitrary base $S$ as soon as a satisfactory theory of generalized intermediate Jacobians has been worked out. This theory has been worked out in case the singular $V_{\bar{s}}(\bar{s} \in \bar{S}-S)$ have ordinary double points as their only singularities (cf. $\$ \$ 15,16$ of the author's paper $O n$ the periods of certain rational integrals, Ann. of Math., November, 1969).

There is one final geometric application of Theorem 5.3 which we should like to give. For this recall that a cohomology class $\psi \in$ $H^{2 l}(S, R)$ is positive (semidefinite) if

$$
\int_{z} \psi \geqq 0
$$

for all complete, $l$-dimensional subvarieties $Z$ of $S$.

(5.12). Theorem. Let $f: X \rightarrow S$ be an algebraic family of algebraic varieties. Then the Chern classes $c_{l}=c_{l}\left(R_{f_{*}}^{a}\left(\theta_{X}\right)\right)$ of the Leray sheaf $R_{f_{*}}^{a}\left(\theta_{X}\right)$ are positive. In particular, if $S$ is complete curve, then $\int_{S} c_{1}>0$ for $q=1,2$ if the variation of Hodge structure is nontrivial 
6. Global results on variation of Hodge structure. The global information on the behavior of the period map (2.2) near infinity seems thus far to have come principally by the methods of hyperbolic complex analysis, but with reduction theory becoming increasingly important. To explain what we mean, we localize the period mapping around a point of $\bar{S}-S$ (cf. (1.1)) so that we have a horizontal, holomorphic mapping

$$
\Phi: \Delta^{* k} \times \Delta^{n-k} \rightarrow D / \Gamma
$$

where $\Delta, \Delta^{*}$ are a disc, respectively punctured disc in $\boldsymbol{C}$. Now $\Delta^{* k} \times \Delta^{n-k}$ carries the usual Poincare metric, and the Cartan-Killing form on $G$ induces an invariant Hermitian metric $d s_{D}^{2}$ on $D$. From [4] we then find that: the period mapping (6.1) is negatively curved in the sense that, with suitable normalization of constants, we have

$$
K_{D}\left(\Phi_{*}(\pi)\right) \leqq-1,
$$

where $\pi$ is a holomorphic 2-plane given by $\pi=\tau \wedge \bar{\tau}$ for some $(1,0)$ tangent vector $\tau$ to $S, \Phi_{*}(\pi)$ is the induced 2-plane on $D$, and $K_{D}\left(\Phi_{*}(\pi)\right)$ is the holomorphic sectional curvature in $\Phi_{*}(\pi)$ [26].

From (6.2) and the generalizations of the classical Schwarz lemma due to Ahlfors, Chern, Kobayashi, Wu [13], [26], we have [17]:

(6.3). Theorem (Generalized Schwarg Lemma). The period mapping $\Phi: S \rightarrow D / \Gamma$ is both distance and volume decreasing locally near infinity.

One of the main uses of this result is to allow us to prove in general certain statements which were easy in the special case when $D$ is a bounded domain (Hermitian symmetric space). For example, we have (cf. [16] and the references given there):

(6.4). Theorem (Removable Singularity theorem). Let $B$ be an irreducible branch of $\bar{S}-S$ around which the P.-L. transformation $T$ is of finite order. Then the period mapping (2.2) extends holomorphically across $B$.

Assume now that $\Phi: S \rightarrow D / \Gamma$ has been extended across the branches of $\bar{S}-S$ where P.-L. is of finite order. Then from [17] we have

(6.5). Theorem. The image $\Phi(S) \subset D / \Gamma$ is a closed complex analytic set of finite volume.

REMARK. Without assuming that we have extended $\Phi$ across the branches of $\bar{S}-S$ where P.-L. is of finite order, we can only assert that $\Phi(S)$ is a locally closed analytic subvariety of $D / \Gamma$.

Using (6.3) and the methods of reduction theory, Borel has proved 
the eigenvalue part of the monodromy theorem (3.1) and the following

(6.6). Theorem (Borel). In case $D$ is a bounded domain, the period mapping $\Phi: S \rightarrow D / \Gamma$ extends to a holomorphic mapping $\Phi: \bar{S} \rightarrow\{D / \Gamma\}^{*}$ where $\{D / \Gamma\} *$ is the Baily-Borel compactification of $D / \Gamma[10]$.

REMARks. This result applies in case the fibres of $f: X \rightarrow S$ are, for example, curves, $K-3$ surfaces, or cubic threefolds. Borel's proof of (6.6) makes use of the assumption that $\bar{S}-S$ has normal crossings, and so it is not known whether or not (6.6) remains true for other smooth compactifications of $S$ (i.e. it is not known in what sense $D / \Gamma$ is "minimal at infinity"). The proof of (6.6) when the fibres of $f: X \rightarrow S$ are curves is due to Mayer and Mumford, whose used a combination algebro-geometric and hyperbolic-complex-analytic argument (cf. $\$ 13$ below for a proof of their theorem).

\section{Part II. Problems and conjectures}

7. Problems on Torelli-type theorems. The local Torelli problem is the question of deciding when the Hodge structure on $H^{*}\left(V_{s}, C\right)$ separates points in the local moduli space (Kuranishi space) of $V_{s}$. This problem leads to a rather interesting multiplicative problem in cohomology [2], [3] which, in specific cases, usually reduces to a geometric question on linear series. Somewhat surprisingly the local Torelli theorem holds in many examples (e.g. curves (Rauch), nonsingular hypersurfaces in projective space (with the one exception of the cubic surface), hypersurfaces on abelian varieties, and varieties with trivial canonical bundle (essentially due to Andreotti)), and, so far as I know, has yet to fail significantly.

(7.1). Problem*. Find methods to treat the local Torelli theorem. In particular, decide whether it is true or false for simply-connected canonical surfaces (i.e. surfaces with ample canonical bundle).

The global Torelli problem is the question of whether or not the total period matrix (i.e. the polarized Hodge structure on $\bigoplus_{q=0}^{2 n}$ $\left.H^{q}\left(V_{s}, C\right), n=\operatorname{dim}_{C} V_{s}\right)$ characterizes uniquely the polarized algebraic structure of $V_{s}$. This is a really beautiful question about which nothing substantial, other than the case of curves (the affirmative answer in this case being the usual Torelli theorem), seems to be known (cf. the second remark below Problem (7.3)).

The main conjecture, which is due to Andreotti and Weil (cf. [2], [20] and the references given there), deals with polarized $K-3$ surfaces. By definition such surfaces are simply-connected, polarized algebraic surfaces which have a trivial canonical bundle. The period matrix domain for the 2 -forms is the bounded symmetric domain 
$D=\mathrm{SO}(19) \times \mathrm{SO}(2) \backslash \mathrm{SO}(19,2)[2]$, and as mentioned above, the local Torelli theorem is true in this case. In fact, both the local moduli space and period matrix domain are 19 dimensional.

(7.2). Conjecture***. The global Torelli theorem holds for $K-3$ surfaces.

Remarks. It follows from Borel's Theorem 6.6 that the period matrices coming from any maximal algebraic family (e.g. any of the countable sequences of algebraic families of polarized $K-3$ surfaces appearing in Italian algebraic geometry-cf. the Enriques book $L e$ superficie algebriche, Bologna, 1949) form a Zariski open set in $D / \Gamma$. Furthermore, in this case the boundary $\partial(D / \Gamma)=\{D / \Gamma\}^{*}-D / \Gamma$ of $D / \Gamma$ in the Baily-Borel compactification has complex dimension one, and we can identify a family of degenerate $K-3$ surfaces lying over $\partial(D / \Gamma)$ as being certain rational surfaces with an elliptic double curve. There has also been recent work by A. Mayer on $K-3$ surfaces with rational singularities (or duVal singularities); he used these to account for some of the elliptic transformations in $\Gamma$.

Speaking personally, it seems to me that the most promising avenue of approach to (7.2) is some sort of direct construction of the function field of a $K-3$ from its period matrix. This might be essentially a uniformization question (cf. \$12 below).

I should like to also put forth the problem of the global Torelli theorem for nonsingular cubic threefolds (cf. [2] and $\$ 23$ of the author's paper On periods of certain rational integrals, Ann. of Math., November, 1969).

(7.3). Problem. ${ }^{* *}$ Is the global Torelli theorem true for nonsingular cubic threefolds?

REMARK. I only put two asterisks here because of the following:

(i) the cubic threefold can most likely be reconstructed from the Fano surface of lines lying in it;

(ii) this Fano surface has irregularity $h^{1,0}=5$ and should be embedded in its Albanese; and

(iii) if this were the case, then the methods from the theory of curves might apply.

REMARK ADDED IN PROOF. I would like to call attention to the recent paper Periods of a moduli space of bundles on curves, Amer. J. Math. 90 (1968) by Mumford and Newstead which gives an interesting global Torelli theorem.

8. Problems on local monodromy and variation of Hodge structure. (a) In this paragraph we shall localize the situation $\bar{f}: \bar{X} \rightarrow \bar{S}$ of $\S 1$ over a disc $\Delta$ which meets a single irreducible branch of $\bar{S}-S$ 
transversely. Thus we assume we have the situation $f: \bar{Y} \rightarrow \Delta$ where

(i) $\Delta C C$ is an ordinary disc around the origin and with holomorphic coordinate $s$;

(ii) $\bar{Y}$ is smooth and the geometric fibres $V_{s}=f^{-1}(s)$ are all connected;

(iii) $f$ is proper and its restriction to $Y=\bar{Y}-V_{0}$ is smooth; and

(iv) there is a distinguished embedding $\bar{Y} \subset P_{N}$.

We let $\Delta^{*}=\Delta-\{0\}$ be the punctured disc and select a base point $s_{0} \in \Delta^{*}$. Denote by $T: H^{q}\left(V_{s_{0}}, C\right) \rightarrow H^{q}\left(V_{s_{0}}, C\right)$ the local P.-L. transformation (§3). The following is the local invariant cycle problem:

(8.1). Conjecture**. Let $\psi \in H^{q}\left(V_{s_{0}}, C\right)$ be a local invariant cohomology class (i.e. $T \psi=\psi)$. Then there is a class $\bar{\Psi} \in H^{q}(\bar{Y}, \mathbf{C})$ with $\bar{\Psi} \mid V_{s_{0}}=\psi$.

Remarks. It is trivial that there exists $\Psi \in H^{q}(Y, C)$ with $\Psi \mid V_{s_{0}}$ $=\psi$. However, it has thus far been amazingly difficult to prove 8.1, and the special cases which have been checked (essentially curves and some special surfaces-cf. §15) suggest that some sort of Hodge theory is needed so that the result (if true) is probably not topological. The global form of the conjecture is due to Deligne (3.6). It is not obvious, but would probably be the case, that the local version would imply the global one.

The principal motivation for the local invariant problem is the question of describing the local vanishing cycles in terms of the P.-L. transformation $T$. By definition, the local vanishing cycle group is the kernel of the inclusion $H_{p}\left(V_{s_{0}}, \boldsymbol{C}\right) \rightarrow H_{p}(\bar{Y}, \boldsymbol{C})$. An affirmative answer to (8.1) would give this vanishing cycle group as $(T-I) \cdot H_{p}\left(V_{s_{0}}, C\right)$.

(b) The next problem is a question raised by Brieskorn and related to me by Deligne. Assume that we are given the situation $f: \bar{Y} \rightarrow \Delta$ as above, but where the fibres $V_{s}$ are now only assumed to be local analytic hypersurfaces in an open set $U \subset C^{n+1}$, which we may take to be given by

$$
f\left(z_{1}, \cdots, z_{n+1}\right)=s
$$

where $f$ is a suitable analytic function of $z_{1}, \cdots, z_{n+1}$. We assume that the $V_{s}$ are nonsingular for $s \in \Delta^{*}$ and that $V_{0}$ has an isolated singular point at the origin in $\boldsymbol{C}^{n+1}$. This is the situation studied extensively by Pham, Brieskorn, et al., and reported on in Milnor's book [8], where also numerous examples are given. We let $M_{\text {s }}$ $=V_{s} \cap B$ where $B$ is a small ball around the origin in $C^{n+1}$. It may be assumed that the $M_{s}\left(s \in \Delta^{*}\right)$ are smooth, real $2 n$-manifolds with boundary, and we consider the cohomology group $H^{n}\left(M_{s_{0}}, Q\right)$ and corresponding P.-L. transformation $T$ [8]. 
(8.2). Problem. Is the P.-L. transformation $T: H^{n}\left(M_{s_{0}}, Q\right)$ $\rightarrow H^{n}\left(M_{s_{0}}, Q\right)$ of finite order?

REMARK. Grothendieck has verified that the eigenvalues of $T$ are roots of unity in this case-cf. [19].

(c) In this problem we return to the usual situation $f: \bar{Y} \rightarrow \Delta^{*}$ where $f$ is proper as in $\S 8(\mathrm{a})$ above.

(8.3). Problem*. Complete the proof, which is outlined in the remark below, of the eigenvalue statement of the monodromy theorem so as to also get the result about elementary divisors.

The solution to (8.3) along the lines proposed in the second remark below would lead to a solution of the following, which is implied by the Hodge conjecture [6]:

(8.4). Conjecture*. If the Hodge numbers $h^{a, b}=\operatorname{dim} H^{a, b}\left(V_{\varepsilon}\right)$ satisfy:

$$
\begin{aligned}
h^{2 p+1,0} & =\cdots=h^{p+1+s, p-s}=0 & & \text { where } q=2 p+1, \\
h^{2 p, 0} & =\cdots=h^{p+s, p-s}=0 & & \text { where } q=2 p,
\end{aligned}
$$

then $\left(T^{N}-I\right)^{r+1}=0$ where

$$
\begin{aligned}
r & =2 s-1 & & \text { in case } q=2 p+1 \\
& =2 s-2 & & \text { in case } q=2 p .
\end{aligned}
$$

REMARK. We can state this conjecture verbally as follows: If the Hodge numbers for the group $H^{q}\left(V_{s_{0}}, C\right)$ start to vanish from the ends, then we should be able to accordingly lower the index of nilpotency in the monodromy Theorem 3.1.

REMARK. An outline of the proof of this problem-conjecture might be as follows: Write $D=H \backslash G$ where $G$ is a real, simple Lie group and $H=H_{1} \times \cdots \times H_{s} \times Z(H)$ is a compact subgroup ( $\$ 2$, (d)), the $H_{j}(j=1, \cdots, s)$ being the simple factors of $H$ and $Z(H)$ being the center of $H$. The point should be that the number $s$ of simple factors of $H$ is the same as the number $s$ in the Conjecture 8.4.

In more detail, we restrict the period mapping (2.2) to the base space of our situation $f: Y \rightarrow \Delta^{*}$ and then lift to universal coverings to obtain a holomorphic mapping

$$
\Phi: H \rightarrow D,
$$

where $H$ is the usual upper-half-plane of all $z=x+i y, y>0$. The mapping satisfies:

(8.6). $\Phi$ is horizontal and $\Phi(z+1)=\Phi(z) \cdot T$ where $T$ is the P.-L. transformation. 
Since $T$ is an integral matrix, the eigenvalue statement will follow from Kronecker's theorem if we can show that the eigenvalues are all of absolute value one (this was pointed out to me by Weil). Having done this we would then want a bound on the index of nilpotency of $T^{N}-I$. Write $T=T_{s} \cdot T_{u}$ (Chevalley decomposition) where $T_{s}$ is semisimple, $T_{u}$ is unipotent, and $T_{s} T_{u}=T_{u} \cdot T_{s}$. First we must show that $T_{s}$ is elliptic; this should be done directly using (8.6) and (6.3), but in any case it is true. Assuming now that $T_{s}=I$, we write $T_{u}=\exp \gamma$ for $\gamma$ in the Lie algebra of G. Using (6.3) we will find an estimate on the norm, with respect to $d s_{D}^{2}$, of the infinitesimal linear transformation $\gamma$ acting on $D$. Taken with suitable information about $d s_{D}^{2}$, we should get (8.4). The point of this approach is that all the calculations can be done in the Lie algebras, and doing this ought to be a manageable problem.

9. Questions on compactification and the behavior of periods at infinity. (a) The discussion in this section was given in preliminary form in [3]. The present reformulation and clarification owes much to discussions with Borel.

Let

$$
\begin{aligned}
X & \subset \bar{X} \\
f \downarrow & \downarrow \bar{f} \\
S & \subset \bar{S}
\end{aligned}
$$

be a situation as described in $\S 1$ and denote by $\Gamma \subset \operatorname{Aut}\left(H^{q}\left(V_{s_{0}}, C\right)_{0}\right)$ the arithmetic group leaving the lattice $H^{q}\left(V_{s_{0}}, Z\right) \cap H^{q}\left(V_{s_{0}}, Q\right)_{0}$ invariant. Denote by $\Phi: S \rightarrow D / \Gamma$ the corresponding period mapping (2.2). Following the lead of Theorem 6.6, we should like to be able to find an analytic space $\{D / \Gamma\}^{*}$ which has $D / \Gamma$ as a Zariski open subset, and which has the property that the period mapping extends to a holomorphic mapping $\Phi: \bar{S} \rightarrow\{D / \Gamma\}^{*}$ for all situations (9.1) (where it is still assumed that $\bar{S}-S$ has normal crossings).

Before stating a formal conjecture, we want to comment about the boundary components [10] of $D \subset \check{D}$ (cf. (c) in §4). Namely, a boundary component of $D$ in its dual $\check{D}$ is, as a tentative definition, a locally closed complex submanifold $F$ of $\check{D}$ which satisfies the conditions

(i) $F$ is contained in $\partial D=\bar{D}-D$;

(ii) $F$ is itself a homogeneous complex manifold which is the orbit of a suitable parabolic subgroup $N(F)$ (called the normalizer of $F$ ) of $G$ under the natural action of $G$ on $\partial D$; and

(iii) if $Z(F)$ is the set $\{g \in N(F)$ such that $g \cdot \Omega=\Omega$ for all $\Omega \in F\}$ is the centralizer of $F$, 
then $F=G_{F} / H_{F}$ where $G_{F}=Z(F) \backslash N(F)$ is a real semisimple Lie group and $H_{F}$ is a compact subgroup. The boundary component $F$ is said to be a rational boundary component if the group $N(F)$ is a $Q$-subgroup of $G$ (cf. [10]). Examples of these concepts are given in $\$ 13$.

(9.2). Conjecture**. There exists a separated, locally compact topological space $\{D / \Gamma\} *$ with the following properties:

(i) $\{D / \Gamma\}^{*}$ contains $D / \Gamma$ as an open, dense subset;

(ii) set-theoretically $\{D / \Gamma\} *$ is the disjoint union

$$
D / \Gamma \cup\left\{\bigcup_{j=1}^{m} F_{j} / \Gamma_{j}\right\}
$$

where the $F_{j}$ are different rational boundary components of $D$ and $\Gamma_{j}=\Gamma \cap N\left(F_{j}\right)$ acts properly discontinuously on $F_{j}$;

(iii) the induced topology on $F_{j} / \Gamma_{j}$ is the natural topology there; and

(iv) a horizontal, holomorphic mapping $\Phi: S \rightarrow D / \Gamma$ extends to a continuous mapping $\Phi: \bar{S} \rightarrow\{D / \Gamma\}^{*}$.

(9.3). Remarks. We should call such a $D / \Gamma$ a partial compactification of $D / \Gamma$, and we should think of $\{D / \Gamma\}^{*}$ as giving a (topological) compactification of $D / \Gamma$ with respect to the holomorphic mappings which arise in algebraic geometry by taking period mappings. In fact, it should be observed that we have made no assertion that either

(i) $\{D / \Gamma\} *$ is a compact topological space, or

(ii) $\{D / \Gamma\} *$ is an analytic space.

Both of these points deserve comment.

Regarding the first, it was pointed out to me by Borel that it seems quite unlikely that there will be a natural (i.e. functorial) compactification of $D / \Gamma$ as an analytic space. Rather, what Borel suggested was that, at best, we should hope for a partial compactification as described in (9.2). It turns out that both the impossibility of a full compactification and the possibility of such a partial compactification receive support from the theory of intermediate Jacobians [2], [3], [23], and I should like to explain this.

The idea is that the theory of intermediate Jacobians has provided a very useful and simpler "abelian" analogue of the period mappings. This being the case, it is natural to see what happens to compactification questions here.

To be more explicit, we take the localized situation $\bar{f}: \bar{Y} \rightarrow \Delta$ of $\bar{f}: \bar{X} \rightarrow \bar{S}$ as was done in $\S 8\left(\right.$ a). Denote by $\Sigma\left(V_{s}\right)$ the group of algebraic cycles of codimension $q$ on $V_{s}$ which are algebraically equivalent to zero there. In case $s=0$ we should, at least as a first approximation, take cycles on $V_{0}$ which have compact support outside the singular 
locus of $V_{0}$ and which are algebraically equivalent to zero there. In any event, for $s \neq 0$, there is a holomorphic mapping, the Abel-Jacobi mapping

$$
\Psi_{s}: \Sigma\left(V_{s}\right) \rightarrow T\left(V_{s}\right)
$$

where $T\left(V_{s}\right)$ is the intermediate Jacobian for cycles of codimension $q$ on $V_{s}[2],[3]$. The map $\Psi_{s}$ has differential restrictions of the same nature as the infinitesimal period relation (2.1) (cf. [2]), and, as mentioned above, gives a simplified model of the period mapping. Now let us assume that, as $V_{s} \rightarrow V_{0}$, the intermediate Jacobian $T\left(V_{s}\right)$ tends somehow to a generalized intermediate Jacobian $T\left(V_{0}\right)$. For example we could take $V_{s}$ to be the product of 3 elliptic curves each acquiring an ordinary double point (in this case we take $q=2$ ). Contrary to the classical case when $T\left(V_{s}\right)$ is a Picard or Albanese variety, it need not happen that $T\left(V_{0}\right)$ has a compactification as an analytic space and which fits nicely into the complex analytic family $\left\{T\left(V_{s}\right)\right\}_{s \in \Delta *}$. The reason for this is that, if we could so compactify $T\left(V_{0}\right)$, then by the monodromy Theorem 3.1 (Clemens' proof [14] works for analytic spaces), we would have $\left(T^{N}-I\right)^{2}=0$ where $T: H^{1}\left(T\left(V_{s}\right), C\right)$ $\rightarrow H^{1}\left(T\left(V_{s}\right), \mathrm{C}\right)$ is the P.-L. transformation. But $H^{1}\left(T\left(V_{s}\right), \mathrm{C}\right)$ $=H_{2 q+1}\left(V_{s}, \mathrm{C}\right)$ so the best we can expect is $\left(T^{N}-I\right)^{2 q+1}=0$ (e.g. the above example of the product of elliptic curves). On the other hand, it seems quite reasonable to expect, and can be checked in easy exsmples such as the one given above, that there is a natural partial aompactification $\left\{T\left(V_{0}\right)\right\}^{*}$ of $T\left(V_{0}\right)$ so that if a cycle $Z$ in $\Sigma\left(V_{0}\right)$ cpecializes to a cycle $Z_{0}$ on $V_{0}$ which may meet the singular locus of $V_{0}$, then $\Psi_{0}(Z)$ will specialize to a point $\Psi_{0}\left(Z_{0}\right)$ in $\left\{T\left(V_{0}\right)\right\}^{*}$. In other words, the analogue of (9.2) in the simpler case of intermediate Jacobians can be checked in some examples and seems plausible in general.

As to the second point in Remark 9.3, we will venture the

(9.5). Conjecture***. Assuming (9.2), the compactification $D / \Gamma$ has the structure of an analytic space such that

(i) $D / \Gamma$ is a Zariski open subset, and

(ii) the extended mappings $\Phi: \bar{S} \rightarrow\{D / \Gamma\} *$ in (iii) of (9.2) will be holomorphic.

The reason for putting three asterisks on (9.5) is that, whereas one can see some possible approaches to (9.2), the analytic structure on $\{D / \Gamma\}^{*}$ has in the past only been possible through the use of some global functions or automorphic forms on $D / \Gamma$ (cf. [10]; the reason for this is because neighborhoods of points at infinity in $\{D / \Gamma\}^{*}$ $-D / \Gamma$ are in some sense quite "large"), and this theory is completely 
missing in the non-Hermitian symmetric case (cf. [4] and \$10 below). In fact, the only real reason for venturing the Conjecture 9.5 is some faith in the global theory of moduli.

(b) Let $f: \bar{Y} \rightarrow \bar{\Delta}$ be a localization, as in $\S 8($ a), of $f: \bar{X} \rightarrow \bar{S}$ around an irreducible branch of $\bar{S}-S$. If the Conjecture (9.2) is to hold, then it should be the case that the Hodge structure of $H^{q}\left(V_{s}, C\right)$ tends to some definite "object" whose parameter space is the rational boundary component $F_{j}$ which contains $\lim _{s \rightarrow 0} \Phi\left(V_{s}\right) \in F_{j} / \Gamma_{j}$ (using the notation of (9.2)). Examples (cf. §7, §13, §14) suggest that this limit object should be a "weighted Hodge structure"; i.e., the $\operatorname{limit} \lim _{s \rightarrow 0}$ $H^{a}\left(V_{s}, \mathbf{C}\right)$ should in first approximation look like the Hodge structure on a vector space $H^{q}\left(W_{0}, \mathbf{C}\right) \oplus \cdots \oplus H^{1}\left(W_{q-1}, \mathbf{C}\right) \oplus H^{0}\left(W_{q}, \mathbf{C}\right)$ where $W_{0}, \cdots, W_{q}$ are nonsingular algebraic varieties, and where the piece $H^{k}\left(W_{q-k}, \mathbf{C}\right)$ is thought of as having weight $k$.

The precise conjecture here is due to Deligne, who arrived at the formulation given below using his and Grothendieck's yoga of "weights on the cohomology of singular or noncomplete algebraic varieties," this yoga arising by translating back to the complex cohomology some consequences of the Weil conjectures for varieties defined over finite fields.

Before giving this conjecture, we need to give a few preliminary explanations. If $E$ is a complex vector space having a conjugation $e \rightarrow \bar{e}(e \in E)$, then a Hodge structure of weight $k$ on $E$ is given by a filtration

$$
F^{0} \subset F^{1} \subset \cdots \subset F^{k}=E
$$

with the property that the mapping

$$
F^{p} \oplus \bar{F}^{k-p-1} \rightarrow E
$$

is, for each $p=0, \cdots, k$, an isomorphism. This is the same as giving a direct sum decomposition

$$
E=\bigoplus_{p+q=k} E^{p, q}, \quad E^{p, q}=\bar{E}^{q, p} .
$$

If $E$ is defined over $Q$, and if we are given a rational bilinear form $Q: E \otimes E \rightarrow \boldsymbol{C}$ such that $Q\left(e, e^{\prime}\right)=(-1)^{k} Q\left(e^{\prime}, e\right)\left(e, e^{\prime} \in E\right)$, then there is the obvious conjugation on $E$ and we have the notion of a polarized Hodge structure of weight $k$ on $E$, which is given by a filtration (9.6) such that (9.7) and the two Hodge bilinear relations [2], [6], [9] are satisfied. Referring to (9.8) these relations are:

$$
\begin{aligned}
& Q\left(E^{p, q}, \bar{E}^{p^{\prime}, q^{\prime}}\right)=0 \quad \text { if } p \neq p^{\prime}, \quad q \neq q^{\prime} ; \\
& (-i)^{k}(-1)^{p} Q\left(E^{p, q}, \bar{E}^{p, q}\right)>0 .
\end{aligned}
$$


We now consider the local system $R_{f_{*}}^{q}(\mathbf{C})=R_{f_{*}}^{q}(\boldsymbol{Q}) \otimes C$ associated to a localized situation $f: Y \rightarrow \Delta^{*}$ as in $\S 8($ a). The P.-L. transformation $T$ acts on each fibre $R_{f_{*}}^{q}(\mathbf{C})_{s}=H^{q}\left(V_{s}, \mathbf{C}\right)$, and we will assume that $T$ is already unipotent so that $(T-I)^{q+1}=0$.

Let $E=R_{f_{*}}^{q}(\mathbf{C})_{s}$ be a typical fibre, which we consider as a complex vector space defined over $\boldsymbol{Q}$ and which has a rational bilinear form

$$
Q: E \otimes E \rightarrow C, \quad Q(v, w)=(-1)^{q} Q(w, v) .
$$

The first thing we want to do is to use

$$
\log T=(T-I)-(T-I)^{2} / 2+\cdots+(-1)^{q+1}(T-I)^{q} / q
$$

to put on $E$ the structure resembling that of the cohomology ring on the topological manifold underlying a $q$-dimensional Kähler manifold, and where $\log T$ plays the role analogous to the Lefschetz-Hodge $L$ operator [9].

To do this we define the Picard-Lefschetz filtration

$$
W^{0} \subset \cdots \subset W^{2 q}=E
$$

as being the unique rational filtration on $E$ such that $\log T\left(W^{k}\right)$ $C W^{k-2}$, and the induced map

$$
(\log T)^{k}: W^{q+k} / W^{q+k-1} \rightarrow W^{q-k} / W^{q-k-1}
$$

is an isomorphism. Thus, as mentioned before, $\log T$ is to be similar to the " $L$ " in Kähler geometry, except of course that $\log T$ decreases indices by two instead of raising by two.

Before continuing, let us show how to construct the P.-L. filtration. Because of (9.12) when $k=q$ we must have

$$
\begin{aligned}
W^{2 q-1} & =\text { kernel of }(\log T)^{q} \text { on } E, \quad \text { and } \\
W^{0} & =\text { image of }(\log T)^{q} .
\end{aligned}
$$

Having defined $W^{2 q-1}$ and $W^{0}$ in this way, we may replace $E$ by $E^{\prime}=W^{2 q-1} / W^{0}$ on which $\log T: E^{\prime} \rightarrow E^{\prime}$ is well defined and now satisfies $(\log T)^{q}=0$. Because of $(9.12)$ again we must have

$$
\begin{aligned}
W^{2 q-2} & =\text { inverse image in } W^{2 q-1} \text { of } \operatorname{ker}(\log T)^{q-1} \text { on } E^{\prime}, \text { and } \\
W^{1} & =\text { inverse image in } W^{2 q-1} \text { of } \operatorname{im}(\log T)^{q-1} \text { in } E^{\prime} .
\end{aligned}
$$

Continuing inductively in this way we will have defined the P.-L. filtration (9.11).

Using the equation

$$
Q(\log T \cdot v, w)+Q(v, \log T \cdot w)=0,
$$


we find that the P.-L. filtration (9.11) is an isotropic or self-dual filtration, which by definition means that we have

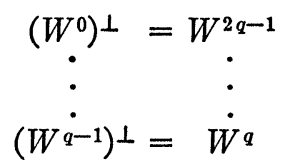

where $\left(W^{k}\right)^{\perp}=\left\{v \in E: Q\left(v, W^{k}\right)=0\right\}$. It follows from (9.14) that there are nonsingular rational bilinear forms

$$
Q_{k}: W^{k} / W^{k-1} \otimes W^{k} / W^{k-1} \rightarrow C, \quad Q_{k}(v, w)=(-1)^{k} Q_{k}(w, v)
$$

such that the maps $(\log T)^{k}$ in (9.12) are isometries. For example we define $Q_{2 q}$ by

$$
Q_{2 q}(v, w)=Q\left(v,(\log T)^{q} w\right), \quad \text { for } v, w \in W^{2 q} / W^{2 q-1} .
$$

These bilinear forms (9.15) are the analogues of the usual ones in Kähler geometry.

There is one final ingredient to put in before giving the conjecture. Namely, we define as usual the primitive part $P_{q+k}$ of $W^{q+k} / W^{q+k-1}$ $(k \geqq 0)$ to be the kernel of $(\log T)^{k+1}: W^{q+k} / W^{q+k-1} \rightarrow W^{q-k-1} / W^{q-k-2}$. Then we have the Lefschetz decomposition

$$
W^{q+k} / W^{q+k-1}=\bigoplus_{l=0}^{[k / 2]}(\log T)^{\imath} P_{q+k+2 l}
$$

as an orthogonal (with respect to $Q_{q+k}$ ) direct sum. This is proved just as in the Kähler case [9].

Deligne's conjecture relates the Hodge filtration of $\$ 1$

$$
F_{s}^{0} \subset \cdots \subset F_{s}^{q}=R_{f *}^{q}(C)_{s}
$$

with the Picard-Lefschetz filtration (9.11) as follows:

(9.17). Conjecture**. The induced filtrations

$$
F_{k}^{p}(s)=W^{k} \cap F_{s}^{p} / W^{k-1} \cap F_{s}^{p}
$$

have the following properties:

(i) the filtration $F_{k}^{0}(s) \subset \cdots \subset F_{k}^{k}(s)=W^{k} / W^{k-1}$ is a Hodge filtration of weight $k$ on $W^{k} / W^{k-1}$;

(ii) the Hodge filtrations in (i) induce polarized Hodge filtrations on the primitive pieces $P_{q+k}$ with the quadratic forms being given by $(9.15)$;

(iii) the $F_{k}^{p}(s)$ vary holomorphically with $s \in \Delta^{*}$; and

(iv) because of (iii) and Theorem 6.4 we see that the Hodge filtration in (i) extends across $s=0$, and we are to then have 


$$
\log T: F_{k}^{p}(0) \rightarrow F_{k-2}^{p-1}(0) .
$$

Remark. From (9.12) and (iv) in the conjecture, we see that the maps

$$
(\log T)^{k}: W^{q+k} / W^{q+k-1} \rightarrow W^{q-k} / W^{q-k-1}
$$

are, at $s=0$, isomorphisms of polarized Hodge structures. It is in this sense that $\lim _{s \rightarrow 0} H^{q}\left(V_{s}, \boldsymbol{C}\right)$ is given by a set of $q+1$ period matrices corresponding to polarized Hodge structures of weight $k$ for $k=0$, $1, \cdots, q$.

(9.18). Remark. For $k=q$ the conjecture says that

$$
Q_{2 q}: W_{Q}^{2 q} / W_{Q}^{2 q-1} \otimes W_{Q}^{2 q} / W_{Q}^{2 q-1} \rightarrow Q
$$

should be positive definite. This is true for $q=1,2$ [14] by using explicit formulae for the P.-L. transformation $T$.

Remark. We will prove (9.17) for $q=1$ in $\$ 13$ below, and will in $\S 14$ give a nontrivial example when $q=2$. At this time the best hope for doing (9.17) in general seems to be to relate it to the compactification question (9.2) so that the methods of group theory and hyperbolic complex analysis become available.

10. Problems on normalization and uniformization of periods. (a) It is of course well known to be the case that the period matrix $\Omega$ of a nonsingular curve can be normalized; that is to say there is a definite nonzero maximal rank minor of $\Omega$, so that we can in fact put $\Omega$ in the usual form $\Omega=\left(I_{n}, Z\right)$ where $Z=X+i Y$ is an $n \times n$ matrix with $Z={ }^{\prime} Z, Y>0$. This implies that whenever we are given the situation $f: X \rightarrow S$ as in $\S 1$ where the fibres $V_{s}$ are curves, then after passing to the universal covering $\tilde{S}$ of $S$ to have the lifted situation $\tilde{f}: \tilde{X} \rightarrow \widetilde{S}$, we will have a definite isomorphism of filtered holomorphic vector bundles over $\tilde{S}$

$$
\psi:\left\{0 \subset \tilde{F}^{0} \subset \tilde{E}\right\} \rightarrow\left\{0 \subset \theta_{\tilde{S}}^{n} \subset \Theta_{\tilde{S}}^{2 n}\right\}
$$

where the image of $\psi$ is given by bounded analytic functions on $\tilde{S}$.

This kind of normalization can equally well be done whenever the period matrix domain $D=H \backslash G$ happens to be a bounded symmetric domain in some $C^{N}$, which is the same as saying that $H=K$ is the maximal compact subgroup of $G$.

In general, there is a topological obstruction to finding such a global coordinate system on $D$. However, for any situation $f: X \rightarrow S$ as above, we may pass to universal coverings to have the lifted period mapping $\tilde{\Phi}: \tilde{S} \rightarrow D$. Then the bundles $\tilde{F}^{p}$ appearing in the lifted Hodge filtrations

$$
0 \subset \tilde{F}^{0} \subset \cdots \subset \tilde{F}^{q}=\tilde{E} \cong \tilde{S} \times H^{q}\left(V_{s_{0}}, C\right)_{0}
$$


are all of the form $\tilde{\Phi}^{*}(L)$ where $L \rightarrow D$ is a homogeneous vector bundle [4]. If we want to find an isomorphism of filtered bundles

$$
\psi:\left\{0 \subset \tilde{F}^{0} \subset \cdots \subset \tilde{F}^{q}\right\} \rightarrow\left\{0 \subset \Theta_{\tilde{S}}^{h_{1}} \subset \cdots \subset{\theta_{\tilde{S}}^{h q}}^{q^{q}}\right\},
$$

then a necessary condition is that the Chern classes of the $\tilde{F}^{p} \rightarrow \tilde{S}$ vanish. This happens as a consequence of the infinitesimal bilinear relation (2.1).

Now, even if we could find an isomorphism $\psi$ as above, there is an analytic obstruction to giving $\psi$ by bounded analytic functions. This obstruction is that the period mapping $\Phi: S \rightarrow D / \Gamma$ should be constant whenever there are no bounded holomorphic functions on $\widetilde{S}$. Using hyperbolic complex analysis, it has been seen to partially be the case that this analytic obstruction also vanishes (cf. [4] and [26] where precise statements are given).

(10.1). Conjecture**. Given $f: X \rightarrow S$, there exists a simultaneous normalization of all of the periods $\Phi\left(V_{s}\right)(s \in S)$. More precisely, suppose we lift the period mapping to the universal coverings to have $\Phi: \tilde{S} \rightarrow D$. Then there exists a natural embedding of the image $\Phi(\tilde{S})$ in a bounded domain $B$ in some $C^{N}$.

Remark. Suppose that the period mapping $\Phi: S \rightarrow D / \Gamma$ satisfies the following conditions:

(i) the differential $\Phi_{*}$ is everywhere injective;

(ii) each of the $P$.-L. transformations around the branches at infinity is of infinite order (cf. (6.4)); and

(iii) the induced map $\Phi_{*}: \pi_{1}\left(S, s_{0}\right) \rightarrow \Gamma$ is injective.

Let $B C D$ be the total inverse image of $\Phi(S)$ in the diagram

$$
\begin{gathered}
D \\
S \stackrel{\Phi}{\rightarrow} D / \Gamma
\end{gathered}
$$

From (6.2), (6.5), and [2] we have:

(10.2). Theorem. $B$ is

(i) topologically a cell,

(ii) strongly Levi-pseudo-convex and therefore a Stein manifold, and

(iii) strongly negatively curved, i.e., has a complete metric $d s_{B}^{2}$ with all holomorphic sectional curvatures satisfying $K_{B}(\pi) \leqq-1(c f .(6.2))$. be?

How much more like a bounded domain can a complex manifold 
(b) The following is, to me, the central remaining foundational question in variation of Hodge structures. It is the problem of finding the analogue of "automorphic functions" for general period mappings $\Phi: S \rightarrow D / \Gamma$ in case $D$ is not a bounded domain. Another way of phrasing the question is to find a natural way of algebracizing the images $\Phi(S) \subset D / \Gamma$ so that the mapping $\Phi: S \rightarrow \Phi(S)$ is a morphism. For example, although it could perhaps be squeezed out of the methods used to prove (6.5), it is certainly not a priori clear to me that the equivalence relation on $S$ given by " $s \sim s^{\prime} \Leftrightarrow$ Hodge structure (with distinguished lattice and polarization) on $H^{q}\left(V_{s}, C\right) \sim$ Hodge structure (with distinguished lattice and polarization) on $H^{q}\left(V_{\boldsymbol{s}^{\prime}}, \mathrm{C}\right)$ " is an algebraic equivalence relation (cf. [2]).

To state the conjecture, we denote by $K \rightarrow D$ the canonical bundle [4] (canonical factor of automorphy) and let $L=\bigoplus_{\mu=0}^{\infty} K^{\mu}$ be the formal direct sum. If $W \subset D / \Gamma$ is a locally closed analytic subvariety, the sheaf $\mathcal{O}(L)$ of holomorphic sections of $L$ is, by definition, the sheaf associated to the presheaf which assigns to each open set $U \subset W$ a $\Gamma$-invariant holomorphic section of $\bigoplus_{\mu=1}^{N} K^{\mu}$ over $\pi^{-1}(U)$, for some $N$ and where $\pi: D \rightarrow D / \Gamma$ is the canonical projection. We observe that there is induced from $d s_{D}^{2}$ an Hermitian metric $d s_{W}^{2}$ on $W$, defined at least at the simple points of $W$, and there is also a pointwise Hermitian metric in $L \rightarrow W[4]$. Thus it makes sense to speak of "holomorphic sections of $L \rightarrow W$ which have prescribed growth conditions at infinity." We do not want to make this too precise, but will only mention that one example of such growth conditions would be to say that "a holomorphic section $\phi \in H^{0}(W, \mathcal{O}(L))$ is square-integrable"

We consider now horizontal, holomorphic mappings $\Phi: S \rightarrow D / \mathrm{T}$ from algebraic varieties $S$ to $D / \Gamma$. Recall from Theorem 6.5 that the image $S_{\Phi}=\Phi(S)$ is a locally closed complex analytic subvariety of $D / \Gamma$. Also the bundle $\Phi^{*} L=L_{\Phi}$ satisfies $g^{*} L_{\Phi^{\prime}}=L_{\Phi}$ whenever we have a commutative diagram

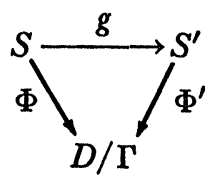

where $\Phi, \Phi^{\prime}$ are horizontal, holomorphic mappings and $g$ is a morphism of algebraic varieties. In particular (10.3) gives a map

$$
g^{*}: H^{0}\left(S^{\prime}, \mathcal{O}\left(L_{\Phi^{\prime}}\right)\right) \rightarrow H^{0}\left(S, \mathcal{O}\left(L_{\Phi}\right)\right) \text {. }
$$


(10.5). Conjecture (Existence of Automorphic forms)**. Given $\Phi: S \rightarrow D / \Gamma$ as above, the image $S_{\Phi}$ of $\Phi$ is an algebraic variety with the following properties:

(i) the holomorphic map $\Phi: S \rightarrow S_{\Phi}$ is a morphism of algebraic varieties; and

(ii) the canonical bundle $K \rightarrow S_{\Phi}$ is ample (i.e. there is an algebraic, projective embedding $S_{\Phi} \subset P_{N}$ given by a subspace of $H^{0}\left(S_{\Phi}, \mathcal{O}\left(K^{\mu}\right)\right)$ for some $\mu>0$ ).

(10.6). REMARK. The conjecture is true in case

(a) $S$ is complete (i.e. compact), or

(b) the period matrix domain $D$ is a bounded symmetric domain (cf. Theorem 6.6 and [10]).

(10.7). REMark. The way one probably should algebracize $S_{\Phi}$ is to prove that there is a graded homogeneous subring $A\left(S_{\Phi}, \mathcal{O}(L)\right)$ of $H^{0}\left(S_{\Phi}, \mathcal{O}(L)\right)$ with the following properties:

(i) $A\left(S_{\Phi}, \mathcal{O}(L)\right)$ is defined by growth conditions at infinity;

(ii) given a diagram (10.3) so that we have $S_{\Phi} \subset S_{\Phi^{\prime}}$, then we should have $A\left(S_{\Phi^{\prime}}, \mathcal{O}(L)\right) \rightarrow A\left(S_{\Phi}, \mathcal{O}(L)\right)$ (i.e. the growth conditions are inherited on subvarieties); and

(iii) $A^{0}\left(S_{\Phi}, \mathcal{O}(L)\right)$ is a finitely generated and related homogeneous coordinate ring whose spectrum is a projective algebraic variety in which $S_{\Phi}$ is canonically embedded as a Zariski open set.

(c) Finally we should like to mention the

(10.6). Problem***. Which Hodge structures come from algebraic varieties?

REMARKS. (i) We should be more precise about what it means for a Hodge structure to "come from an algebraic variety." Certainly we should include all Hodge structures, or equivalently period matrices, which arise from the primitive cohomology of smooth, projective algebraic varieties. However, Grothendieck has commented that we should also include the following type of Hodge structures: Let $V$ be a smooth, complete $q$-dimensional algebraic variety and $Z \subset V \times V$ a $q$-dimensional algebraic cycle. Then the homology class of $Z$ gives a mapping $\phi_{Z}: H^{q}(V, \mathbf{C}) \rightarrow H^{q}(V, \boldsymbol{C})$, where $\phi_{Z}$ is obtained from the Künneth isomorphism

$$
H^{*}(V \times V, \mathbf{C}) \cong \operatorname{Hom}\left(H_{*}(V, \mathbf{C}), H^{*}(V, \mathbf{C})\right) .
$$

The image $\phi_{Z}\left\{H^{q}(V, C)\right\}$ of $\phi_{Z}$ is a sub-Hodge structure of $H^{q}(V, \boldsymbol{C})$, and all such Hodge structures should also be considered as coming from algebraic varieties. It is a consequence of Grothendieck's theory of motives that these Hodge structures will exhaust those that can reasonably be said to arise from algebraic varieties. 
(ii) If the period matrix domain $D$ for a particular type of Hodge structure is a bounded domain, then it is more or less an observation that all Hodge structures $\Omega \in D$ come from algebraic varieties (we are purposely being sloppy about the lattices and discrete subgroups in the problem). On the contrary, if $D$ is not a bounded domain, then it was pointed out by Mumford that the set of $\Omega \in D$ which come from algebraic varieties is a countable union of proper analytic subsets of $D$ (cf. (2.4)), and is thus a set of the 1st category in the Baire sense. Thus the problem has completely changed character and it is anybody's guess as to what is going on.

11. Problems on automorphic cohomology. (a) Associated to a period matrix domain $D$ are two natural objects:

(i) the set of locally liftable, horizontal, holomorphic mappings $\Phi: S \rightarrow D / \Gamma$ from algebraic varieties $S$ to suitable quotien ts of $D$; and (ii) the automorphism group $G$ of $D$ and, in particular, the unitary representations of $G$ which are derived from the complex structure on $D$ [4], [24].

In case $D=K \backslash G$ is a bounded domain, there is a close connection between these objects provided by the theory of automorphic forms (cf. [10], [24] where further references are given). Roughly speaking, the space of square-integrable, holomorphic sections $H_{2}^{0}\left(D, K^{\mu}\right)$ of sufficiently high powers of the canonical bundle gives a nonzero, irreducible, unitary $G$-module which belongs to the discrete series part of $L_{2}(G)$. There is a dense subspace $H_{1}^{0}\left(D, K^{\mu}\right)$ of $H_{2}^{0}\left(D, K^{\mu}\right)$ given by $L_{1}$-holomorphic sections of $K^{\mu} \rightarrow D$, and for such $\phi \in H_{1}^{0}\left(D, K^{\mu}\right)$ and a given arithmetic subgroup $\Gamma$ of $G$ we can form Poincaré and Poincaré-Eisenstein series with $\phi$ to obtain $\Gamma$-invariant holomorphic sections in $H^{0}\left(D / \Gamma, \mathcal{\Theta}\left(K^{\mu}\right)\right.$ ) (cf. [10]). Such automorphic forms can then be used to prove Conjecture (10.5) and, in general, they play a central role in both the geometric and arithmetic theory of moduli.

For general period matrix domains $D$, both $H_{2}^{0}(D, E)$ and $H^{0}(D / \Gamma, \mathcal{O}(E))$ are zero for all homogeneous holomorphic vector bundles $E \rightarrow D$ unless $D$ happens to already be a bounded domain (cf. [4]). However, it has been recently proved by W. Schmid [24] that we do have automorphic cohomology which stands in the same relation to $L_{2}(G)$ as the automorphic forms did in the bounded symmetric domain case. More precisely, Schmid proves that the " $L_{2}$-cohomology group" $H_{2}^{p}\left(D, K^{\mu}\right)=0$ unless $p=q_{0}=\operatorname{dim}_{\mathrm{C}} H \backslash K$, and that $H_{2}^{q_{0}}\left(D, K^{\mu}\right)$ is (at least for $\mu \geqq \mu_{0}$ ) an irreducible unitary $G$-module appearing with preassigned character in the discrete series part of $L_{2}(G)$ (cf. [24] where the undefined terms are explained). Moreover, there is a dense subspace $H_{1}^{q_{0}}\left(D, K^{\mu}\right)$ of $L_{1}$-harmonic forms 
in $H_{2}^{q_{0}}\left(D, K^{\mu}\right)$ and so Poincaré series (and perhaps also suitably defined Eisenstein series?) can be formed as in the bounded domain case [2]. In other words, what we quite naturally are led to is automorphic cohomology $H^{q_{0}}\left(D / \Gamma, \mathcal{O}\left(\boldsymbol{K}^{\mu}\right)\right)$, which by definition is the $\Gamma$-invariant classes in $H^{q_{0}}\left(D, \mathcal{O}\left(K^{\mu}\right)\right)$ (cf. [4] where a discussion of $H^{q_{0}}\left(D / \Gamma, \mathcal{O}\left(K^{\mu}\right)\right)$ is given in case $D / \Gamma$ is compact).

(11.1). Problem**. Relate the automorphic cohomology $H^{q_{0}}\left(D / \Gamma, \mathcal{O}\left(K^{\mu}\right)\right)$ to the horizontal, holomorphic maps $\Phi: S \rightarrow D / \Gamma$ arising from variation of Hodge structures. In particular, can automorphic cohomology be used to construct the automorphic forms requested in (10.7)?

(11.2). Remark. A possible interpretation of the cohomology $H^{q_{0}}\left(D, \mathcal{O}\left(K^{\mu}\right)\right)$ is as follows (cf. [3] and the references given there): Let $Z \subset D$ be the compact, complex analytic subvariety $H \backslash K$ embedded in $D$. Then $\operatorname{dim}_{C} Z=q_{0}$ and $K \rightarrow Z$ is a negative line bundle [2], [4] in the sense of Kodaira. Now $Z$ is also an algebraic subvariety of the homogeneous rational variety $\check{D}$, and so we may consider the Chow variety $\Lambda(Z)$ of all effective algebraic cycles $Z^{\prime}$ on $\check{D}$ which are algebraically equivalent to $Z$ there. Let $\Lambda^{0}(Z)$ be the irreducible component of $\Lambda(Z)$ which contains $Z$ and denote by $\Psi$ the open set of all $Z^{\prime} \in \Lambda^{0}(Z)$ such that $Z^{\prime} \subset D$. It seems likely, but is certainly not proved, that if $D$ is not a bounded domain there will be an embedding

$$
\Psi \subset K_{C} \backslash G_{C}
$$

of $\Psi$ as an open set in the affine homogeneous variety $K_{C} \backslash G_{C}$. (The map $\Psi \rightarrow K_{C} \backslash G_{C}$ would send $Z^{\prime} \in \Psi$ into $K_{C} \cdot g$ where $Z^{\prime}=Z \cdot g$ for a suitable $g \in G_{C}$.) Now $K_{C} \backslash G_{C}$ is an affine variety defined over the rationals $Q$, and the set of real points of $K_{C} \backslash G_{C}$ is just the Riemannian symmetric space $R=K \backslash G$ (cf. (c) in \$2).

Let $E_{\mu} \rightarrow \Psi$ be the holomorphic vector bundle whose fibre $\left(E_{\mu}\right)_{Z^{\prime}}$ over $Z^{\prime} \in \Psi$ is the cohomology group $H^{q_{0}}\left(Z^{\prime}, \mathcal{O}_{Z^{\prime}}\left(K^{\mu}\right)\right)$. Then there is a natural injective mapping

$$
H^{q_{0}}\left(D, \mathcal{O}\left(K^{\mu}\right)\right) \rightarrow H^{0}\left(\Psi, \mathcal{O}\left(E_{\mu}\right)\right)
$$

which serves to faithfully interpret the cohomology $H^{q_{0}}\left(D, \mathcal{O}\left(K^{\mu}\right)\right)$ as holomorphic sections of holomorphic bundle somewhere. Furthermore, we may pass to quotients to have

$$
H^{q_{0}}\left(D / \Gamma, \mathcal{O}\left(K^{\mu}\right)\right) \rightarrow H^{0}\left(\Psi / \Gamma, \mathcal{O}\left(E_{\mu}\right)\right),
$$

and thus interpret the automorphic cohomology. (This procedure is closely related to recent work of Andreotti-Norguet (Annali della 
Scuola Normale di Pisa, §3, 21 (1967), 31-82) on complex manifolds which are partially convex and partially concave.)

There is a very interesting recent result of Schmid and Wells which says that $\Psi$ is a Stein manifold in a special case. Namely, if $D$ $=\operatorname{SO}(4,1 ; R) / U(2)$, the $\Psi$ is given as

$$
\left\{z \in C^{4}, w \in C: z \cdot z=w \cdot w-1,|w|^{2}>|z|^{2}\right\}
$$

where $z \cdot z=\sum_{j=1}^{4}\left(z_{j}\right)^{2},|z|^{2}=z \cdot \bar{z}$, etc. Wells remarks that it seems likely that $\Psi$ will always be Stein, but this is not proved yet.

The trouble with all of this is that it is not the least bit clear what our possible interpretation of automorphic cohomology has to do with period mappings.

(b) Let me give an explicit, and perhaps more accessible, special case of the automorphic cohomology problem (11.1) above. This situation arises in the theory of intermediate Jacobians [2].

Consider a $2 n$-dimensional complex vector space $E$ in which we have a fixed lattice $\Lambda \cong Z^{2 n}$, and on which we have a skew-form $Q: E \otimes E \rightarrow C$ which has the standard matrix

$$
\left(\begin{array}{cc}
0 & I_{n} \\
-I_{n} & 0
\end{array}\right)
$$

on a suitable basis of $\Lambda$. Let $\mathcal{F}$ be the flag manifold of all pairs of subspaces $\Omega=\left[S_{1}, S_{2}\right]$ with $S_{1} \subset S_{2} \subset E, \operatorname{dim} S_{1}=n-q$, and $\operatorname{dim} S_{2}=n$. On $\mathcal{F}$ we take the algebraic submanifold $\check{D}$ of all $\Omega$ with $Q\left(S_{2}, S_{2}\right)=0$ (i.e. $S_{2}$ is isotropic with respect to $Q$ ). The complex, simple algebraic group $\breve{C}$ of all matrices $T$ with $T Q^{t} T=Q$ acts transitively on $\check{D}$, so that $\check{D}$ is a rational, homogeneous projective variety [4]. In $\check{D}$ we take the domain of all $\Omega=\left[S_{1}, S_{2}\right]$ such that

$$
\sqrt{-1} Q\left(S_{2}, \bar{S}_{2}\right)=H=\left(\begin{array}{ll}
H_{11} & H_{12} \\
H_{21} & H_{22}
\end{array}\right)
$$

is a nonsingular Hermitian matrix with signature $(n-q, q)$, and with $H_{11}=\sqrt{-1} Q\left(S_{1}, \bar{S}_{1}\right)$ positive definite. Thus

$$
A H^{t} \bar{A}=\left(\begin{array}{lr}
I_{n-q} & 0 \\
0 & -I_{q}
\end{array}\right)
$$

for some matrix

$$
A=\left(\begin{array}{ll}
A_{11} & 0 \\
A_{21} & A_{22}
\end{array}\right) \text {. }
$$


The domain $D$ is the $G$-orbit of a suitable point in $\check{D}$ where $G$ is the obvious real form of $\breve{G}$. In the usual notation from Lie groups, $D=U(n-q) \times U(q) \backslash \operatorname{Sp}(2 n, R)$.

Given $\Omega \in D, T_{\Omega}=\Lambda \backslash E / S_{2}$ is a complex $n$-torus with a $q$-convex polarization $L_{\Omega} \rightarrow T_{\Omega}[2]$, [3]. We then have $H^{p}\left(T_{\Omega}, \mathcal{O}\left(L_{\Omega}^{\mu}\right)\right)=0$ for $\mu>0, p \neq q$, and also $\operatorname{dim} H^{q}\left(T_{\Omega}, \mathcal{O}\left(L_{\Omega}^{\mu}\right)\right)=n^{\mu}$, just as in the abelian variety case $q=0$. We agree to call $H^{q}\left(T_{\Omega}, \mathcal{O}\left(L_{\Omega}^{\mu}\right)\right)$ the theta cohomology and recall that it can be constructed directly [2]. In the classical case $q=0$, there is a close relation between theta functions and automorphic forms (given by the "theta-nullwerte").

(11.3). Problem*. In the situation just described find the relation between theta cohomology and automorphic cohomology.

REMARK. Let us construct the universal family $J \rightarrow D / \Gamma$ $(\Gamma=\mathrm{Sp}(2 n, \boldsymbol{Z}))$ of complex tori with $q$-convex polarization as follows: Over $D$ we have the universal bundle sequence

$$
0 \rightarrow S \rightarrow E \rightarrow T \rightarrow 0
$$

where $E=D \times E, S$ is the subbundle whose fibre $S_{\Omega}$ is $S_{2}$ where $\Omega=\left[S_{1}, S_{2}\right]$ and where $T$ is the quotient bundle. The projection of $\Lambda$ to $T$ gives an analytic family $\Lambda \backslash T \rightarrow D$ of complex $n$-tori with $T_{\Omega}$ lying over $\Omega \in D$. There is a natural line bundle $L \rightarrow \Lambda \backslash T$ with $L \mid T_{\Omega}$ $=L_{\Omega}$.

Now the modular group $\Gamma$ operates naturally on the situation

$$
\begin{array}{r}
L \stackrel{\pi}{\rightarrow} \Lambda \backslash T \\
\\
\quad \downarrow \tilde{\omega} \\
D
\end{array}
$$

and we may pass to the quotient to have:

$$
\begin{aligned}
\stackrel{\pi}{\rightarrow} & J \\
& \downarrow \tilde{\omega} \\
& D / \Gamma
\end{aligned}
$$

(Here it is convenient to let $\Gamma$ be a suitable congruence subgroup so as not to have fixed points.)

With these explanations, I would guess that the Problem (11.3) is essentially a question of understanding the cohomology

$$
H^{q+p}(\mathcal{J}, \mathcal{O}(\mathcal{L})) \cong H^{p}\left(D / \Gamma, R_{\tilde{\omega}}^{q} \mathcal{O}(\mathcal{L})\right)
$$

in some manner analogous to how one understands automorphic 
forms by knowing about theta-functions and theta-nullwerte.

12. Some general problems on complex analysis and uniformization of algebraic function fields. The questions in this section are not specifically about variation of Hodge structure but are, to some extent, prompted by Theorem 10.2 and by the Torelli problem 7.2 for $K-3$ surfaces.

To state the first question we let $W$ be a smooth, but not necessarily complete, algebraic variety. Given $w \in W$, a good neighborhood of $W$ is defined to be a Zariski open set $U \subset W$ with $w \in U$ and which satisfies:

(i) $\pi_{i}(U)=0$ for $i \geqq 2$;

(ii) $\pi_{i}(U, w)$ is a successive extension of free groups of finite type. It is a theorem of M. Artin that good neighborhoods exist (cf. S.G.A., Cohomologie étale des schémas, 1963-64, Exposé XI).

(12.1). Problem.** Consider a point $w \in W$ and an affine good neighborhood $U$ of $w$ as above. Let $\pi: \widetilde{U} \rightarrow U$ be the universal covering of $U$, so that $\widetilde{U}$ is a Stein manifold which is topologically a cell. Then what do such $\tilde{U}$ look like complex-analytically? Are they domains in $\boldsymbol{C}^{n}\left(n=\operatorname{dim}_{C} \tilde{U}\right)$ ? Does every point $w \in W$ have a good neighborhood $U$ whose universal covering $\tilde{U}$ is a bounded domain?

REMARKs. As evidence that $\tilde{U}$ may be a bounded domain, we mention the following results, which are fairly easy to prove:

(12.2). Theorem (local Picard theorem). Given weW, there exists a good neighborhood $U$ of w such that any holomorphic mapping $f: C^{m} \rightarrow U$ is constant.

(12.3). THEOREM. If $\operatorname{dim} W=2$, then there exist good neighborhoods $U$ such that there is a smooth holomorphic mapping $f: \widetilde{U} \rightarrow \Delta$ of $\tilde{U}$ onto a disc $\Delta$ in $C$ and where each fibre $f^{-1}(t)$ is biholomorphically equivalent to a disc $\Delta$.

(12.4). Problem.*** Assuming in Problem 12.1 that $\tilde{U}$ turns out to be a bounded domain, we consider the Bergman metric $d s^{2}$ on $\tilde{U}$ and fundamental group $\Gamma$ of $U$ acting as isometries on $\tilde{U}$. Then does $U=\tilde{U} / \Gamma$ have finite volume with respect to the Bergman metric on $\tilde{U}$ ? Is the field of rational functions on $U$ given as a subfield of the $\Gamma$-invariant meromorphic functions on $\tilde{U}$ which have suitable growth behaviour at infinity?

\section{Part III. Examples AND SPECIAL CASES}

13. Periods of algebraic curves acquiring singularities. We want to prove a theorem which is based on an unpublished result of Mayer and Mumford. 
(13.1). Theorem. Conjectures $9.2,9.5$, and 9.17 are true when $q=1$.

REMARKs. Conjectures 9.2 and 9.5 shall now be combined and rewritten slightly to read as follows:

$(13.1)^{\prime}$. Let $\bar{f}: \bar{Y} \rightarrow \Delta$ be a localization of $\bar{f}: \bar{X} \rightarrow \bar{S}$ over a disc $\Delta$ which meets transversely an irreducible branch of $\bar{S}-S$ as in $\S 8$ (a). Then the period mapping $\Phi: \Delta^{*} \rightarrow D / \Gamma$ for $q=1$ extends to a continuous mapping $\Phi: \Delta \rightarrow\{D / \Gamma\} *$ into the Satake compactification [10], [25].

In fact, for $q=1$ the period matrix domain is the Siegel upper-halfplane (cf. $\$ 13(\mathrm{c})$ below and [25]) of all $n \times n$ matrices $Z=X+i Y$ which satisfy

$$
Z={ }^{t} Z, \quad Y>0,
$$

and $\Gamma$ is a so-called paramodular group. In this case the compactification of $D / \Gamma$ as a normal analytic space of the sort asked for in 9.2 and 9.5 was done by Satake and Baily [25]. Our proof can be generalized a little to show that the period map extends to a holomorphic map $\bar{\Phi}: \bar{S} \rightarrow\{D / \Gamma\} *$ (cf. Theorem 6.6).

The proof will be given in three steps in sections (a)-(c) below. We may assume that the P.-L. matrix $T$ is already unipotent and thus satisfies $(T-I)^{2}=0$.

(a) Asymptotic form of the period matrix. What we want to prove is:

(13.3). Proposition. There exists a rational basis $\delta_{1}, \cdots, \delta_{2 n}$ of $H_{1}\left(V_{8}, Q\right)$ with respect to which the intersection form Q, P.-L. matrix $T$, and period matrix $Z(s)$ have the following form:

$$
\begin{gathered}
Q=\left(\begin{array}{ccc}
0 & I_{n} \\
-I_{n} & 0
\end{array}\right) \\
T=\left(\begin{array}{llll}
I_{m} & 0 & \Lambda & 0 \\
0 & I_{n-m} & 0 & 0 \\
0 & 0 & I_{m} & 0 \\
0 & 0 & 0 & I_{n-m}
\end{array}\right), \quad \Lambda={ }^{t} \Lambda \text { and } \Lambda>0 \\
\left.Z(s)=\frac{\log s}{2 \pi i}\left(\begin{array}{ll}
\Lambda & 0 \\
0 & 0
\end{array}\right)+\left(\begin{array}{cc}
Z_{11}(s) & Z_{12}(s) \\
{ }^{t} Z_{12}(s) & Z_{22}(s)
\end{array}\right)\right\} n-m
\end{gathered}
$$

where $Z_{i j}(s)$ is holomorphic in $\Delta$ and $\operatorname{Im} Z_{22}(s)>0$ there.

Proof. We will first prove (13.4) and (13.5) (except for the assertion $\Lambda>0$ in (13.5)), which are purely algebraic results. Since $(T-I)^{2}$ $=0$ we may find a basis $x_{1}, \cdots, x_{m} ; y_{1}, \cdots, y_{m} ; z_{1}, \cdots, z_{2 n-2 m}$ for the rational homology group $H_{1}\left(V_{s}, Q\right)$ such that 


$$
\begin{array}{ll}
T x_{j}=x_{j}+y_{j} & j=1, \cdots, m ; \\
T y_{j}=y_{j} & j=1, \cdots, m ; \quad \text { and } \\
T z_{\alpha}=z_{\alpha} & \alpha=1, \cdots, 2 m-2 n .
\end{array}
$$

Since $Q\left(x_{i}, y_{j}\right)=Q\left(T x_{i}, T y_{j}\right)=Q\left(x_{i}+y_{i}, y_{j}\right)$, we have $Q\left(y_{i}, y_{j}\right)=0$ tor all $i, j$. Similarly $Q\left(y_{i}, z_{\alpha}\right)=0$ for all $i, \alpha$, and from

$$
Q\left(x_{i}, x_{j}\right)=Q\left(x_{i}+y_{i}, x_{j}+y_{j}\right)
$$

it follows that $Q\left(y_{i}, x_{j}\right)+Q\left(x_{i}, y_{j}\right)=0$. Thus the intersection matrix has the form

$$
\left.Q=\left(\begin{array}{rrr}
0 & A & 0 \\
-{ }^{t} A & B & C \\
0 & -{ }^{t} C & D
\end{array}\right)^{\}}\right\}_{z_{\alpha} ;}^{\} y_{j}} \quad A={ }^{\prime} A .
$$

Now let $M=\frac{1}{2}^{t}\left(A^{-1} B\right)$ and $x_{j}^{\prime}=x_{j}+\sum_{i=1}^{m} M_{j}^{i} y_{i}$. Then $Q\left(x_{j}^{\prime}, x_{i}^{\prime}\right)$ $=B_{j i}+\sum_{k} M_{j}^{k} A_{k i}-\sum_{l} M_{i}^{l} A_{l j}=0$, and this eliminates $B$ in (13.8). Similarly, we can eliminate $C$. Relettering our basis, we may assume that (13.7) holds and that

$$
\left.Q=\left(\begin{array}{rll}
0 & A & 0 \\
-{ }^{t} A & 0 & 0 \\
0 & 0 & B
\end{array}\right)\right\} \begin{aligned}
& \} y_{j} \\
& \gamma_{j}
\end{aligned} \quad A={ }^{t} A, \quad B+{ }^{t} B=0 .
$$

If we make a substitution $x_{i}^{\prime}=\sum_{k=1}^{m} E_{i}^{k} x_{k}, y_{i}^{\prime}=\sum_{k=1}^{m} F_{i}^{k} y_{k}$, then in (13.9) the new $A^{\prime}=E A^{t} F$. Thus we can choose $E, F$ so that $A=I$ in (13.9) (of course (13.7) no longer holds). We may also choose the $z_{\alpha}$ so that

$$
B=\left(\begin{array}{ll}
0 & I_{n-m} \\
-I_{n-m} & 0
\end{array}\right)
$$

Then if we take our final basis $\delta_{1}, \cdots, \delta_{2 n}$ for $H_{1}\left(V_{s}, Q\right)$ in the order $y_{1}, \cdots, y_{m} ; z_{1}, \cdots, z_{n-m} ; x_{1}, \cdots, x_{m} ; z_{n-m+1}, \cdots, z_{2 n-2 m}, Q$ will have the form (13.4) and $T$ will have the form (13.5), except we cannot yet say that $\Lambda>0$.

We now will prove (13.6) and the $\Lambda>0$ part of (13.5). For $q=1$ the Hodge filtration is just $F_{s}^{0} C E_{s}=H^{1}\left(V_{s}, C\right)$, where $\operatorname{dim} F_{s}^{0}=n$ and where the Riemann bilinear relations (cf. (13.21) and (13.22) below)

$$
Q\left(F_{s}, F_{s}\right)=0, \quad i Q\left(F_{s}, \bar{F}_{s}\right)>0
$$

are satisfied. It follows from Theorem 4.1 (cf. p. 35 in [3]) that we can find a single-valued holomorphic basis $\omega_{1}(s), \cdots, \omega_{n}(s)$ for $F_{s}$ on the punctured disc $\Delta^{*}$ such that the periods $\Omega_{\alpha \rho}(s)=\left\langle\omega_{\alpha}(s), \delta_{\rho}(s)\right\rangle=$ 
$\int_{\delta_{\rho}(s)} \omega_{\alpha}(s)$ have the form $f(s)+l(s) g(s)$ where $f, g$ are holomorphic on the whole disc and $l(s)=\log s / 2 \pi i$. It follows that the period matrix

$$
\Omega(s)=\Omega_{0}(s)+l(s) \Omega_{1}(s), \quad l(s)=\log s / 2 \pi i
$$

where $\Omega_{0}(s), \Omega_{1}(s)$ are holomorphic $n \times 2 n$ matrices. Since analytic continuation around the origin changes $\Omega(s)$ into $\Omega(s) T$, we may rewrite (13.10) as

$$
\Omega(s)=\Omega_{0}(s)+l(s) \Omega_{0}(s)(T-I),
$$

where $\Omega_{0}(s)$ is holomorphic and $T$ is given by (13.5). Writing $\Omega(s)$ $=\left(\Omega_{1}(s), \Omega_{2}(s)\right)$ where $\Omega_{1}(s)$ and $\Omega_{2}(s)$ are each $n \times n$ matrices, it follows from (13.11) that $\Omega_{1}(s)$ is holomorphic, and it follows from the Riemann bilinear relations that $\operatorname{det} \Omega_{1}(s) \neq 0$ for $s \neq 0$. Thus we may replace $\Omega(s)$ by $\Omega_{1}(s)^{-1} \Omega(s)=\left(I_{n}, Z(s)\right)$ where

$$
Z(s)=\left(\begin{array}{ll}
Z_{11}(s) & Z_{12}(s) \\
{ } Z_{12}(s) & Z_{22}(s)
\end{array}\right)+l(s)\left(\begin{array}{cc}
\Lambda & 0 \\
0 & 0
\end{array}\right), \quad l(s)=\frac{\log s}{2 \pi i} .
$$

In (13.12) the $Z_{i j}(s)$ are meromorphic matrices and $Z(s)$ satisfies the Riemann bilinear relations (13.2).

We want to prove that the $Z_{i j}(s)$ are holomorphic functions of $s$ in the whole disc $\Delta$. Since $Z_{22}(s)={ }^{t} Z_{22}(s)$ and $\operatorname{Im} Z_{22}(s)>0, Z_{22}(s)$ may be thought of as a mapping into a Siegel upper-half-plane of genus $n-m$, which is a bounded domain in $C^{N}(N=(n-m)(n-m+1) / 2)$ [25]. Thus by the Riemann extension theorem, $Z_{22}(s)$ is holomorphic in the disc $\Delta$ and $\operatorname{Im} Z_{22}(s)>0$ for all $s \in \Delta$.

We now prove that $Z_{11}(s)$ is holomorphic in $\Delta$ by using induction on the size of the minors of $Z_{11}(s)$. Suppose first that the diagonal entry $z_{j j}(s)=a / s^{k}+\cdots$ for some $a \neq 0, k>0$. We may suppose that $a=1$, and then we will have

$$
\begin{aligned}
1>\left|\exp \left(i\left(z_{j j}(s)+\frac{\log s}{2 \pi i} \lambda_{j j}\right)\right)\right| \\
=\left|\exp \left(\frac{i}{s^{k}}\right)\right| \cdots\left|\exp \left(\frac{s \lambda_{j j}}{2 \pi}\right)\right|
\end{aligned}
$$

for all $0<|s|<\epsilon$. This clearly is a contradiction. Thus all $1 \times 1$ minors of $Z_{11}(s)$ are holomorphic. Assuming that all the principal $l \times l$ minors of $Z_{11}(s)$ have been proved to be holomorphic, then a similar argument to the one just given shows that all principal $(l+1) \times(l+1)$ minors are holomorphic in $\Delta$. Finally, after we have proved that $Z_{11}(s)$ and $Z_{22}(s)$ are holomorphic in (13.12), we can essentially repeat the argument just given to show that $Z_{12}(s)$ is holomorphic in the whole disc $\Delta$. 
It remains to prove that $\Lambda>0$. Write $s=\exp (2 \pi i t), t=\xi+i \eta$ with $\eta>0$. Then we have an expansion

$$
\operatorname{Im} Z(t)=A+e^{-\eta}\{B(t)\}+\eta\left(\begin{array}{ll}
\Lambda & 0 \\
0 & 0
\end{array}\right)
$$

where $A$ is a constant matrix. Thus from (13.14) we have

$$
0<\frac{\operatorname{Im} Z(t)}{\eta}=\left(\begin{array}{ll}
\Lambda & 0 \\
0 & 0
\end{array}\right)+\frac{1}{\eta}\{C(t)\} .
$$

Taking the limit as $\eta \rightarrow \infty$ gives $\Lambda \geqq 0$. Since $\operatorname{det} \Lambda \neq 0$, we have $\Lambda>0$ as required.

(b) Proof of Deligne's conjecture 9.17 for $q=1$. This will be a straightforward application of Proposition 13.4. Namely, using (13.4), (13.5), and (13.6) we can choose a rational basis $\omega_{1}, \cdots, \omega_{2 n}$ for $E_{s}=H^{1}\left(V_{s}, Q\right) \otimes_{Q} C$, and a basis $\phi_{1}, \cdots, \phi_{n}$ for $F_{s}^{0}=H^{1,}{ }^{0}\left(V_{s}, C\right)$, such that we have the following:

$$
Q\left(\omega_{\alpha}, \omega_{n+\alpha}\right)=+1=-Q\left(\omega_{n+\alpha}, \omega_{\alpha}\right) \quad(\alpha=1, \cdots, n)
$$

and all other $Q\left(\omega_{i}, \omega_{j}\right)=0$;

$$
T \omega_{\rho}=\omega_{\rho}+\sum_{\sigma=1}^{m} \Lambda_{\rho \sigma} \omega_{n+\sigma} \quad(\rho=1, \cdots, m), \Lambda={ }^{t} \Lambda \text { and } \Lambda>0,
$$

and all other $T \omega_{j}=\omega_{j}$;

$$
\phi_{\alpha}(s)=\omega_{\alpha}+\sum_{\beta=1}^{n} Z_{\alpha \beta}(s) \omega_{n+\beta}
$$

where $Z(s)$ is given by (13.6).

According to (9.12), the Picard-Lefschetz filtration $W^{0} \subset W^{1} \subset W^{2}$ of $E_{s}$ is to be given by

$$
\begin{aligned}
& W^{1}=\operatorname{ker}(T-I), \\
& W^{0}=\operatorname{im}(T-I) .
\end{aligned}
$$

From (13.16) and (13.18) we see that the successive quotients are:

$$
\begin{aligned}
W^{2} / W^{1} & =\left\{\omega_{1}, \cdots, \omega_{m}\right\}, \\
W^{1} / W^{0} & =\left\{\omega_{m+1}, \cdots, \omega_{n} ; \omega_{n+m+1}, \cdots, \omega_{2 n}\right\}, \text { and } \\
W^{0} & =\left\{\omega_{n+1}, \cdots, \omega_{n+m}\right\},
\end{aligned}
$$

where $\left\{e_{1}, \cdots e_{l}\right\}$ is notation for the vector space with basis $e_{1}, \cdots, e_{l}$. Since $(\log T) \omega_{\rho}=\sum_{\sigma=1}^{m} \Lambda_{\rho \sigma} \omega_{n+\sigma}$, the bilinear pairing $Q_{2}: W^{2} / W^{1} \otimes W^{2} / W^{1} \rightarrow C$ is given by $Q_{2}\left(\omega_{\rho}, \omega_{\sigma}\right)=\Lambda_{\rho \sigma}$, which is rational and positive definite by (13.5). The bilinear pairing $Q_{1}$ on 
$W^{1} / W^{0}$ is given by $Q_{1}\left(\omega_{j}, \omega_{n+j}\right)=+1=-Q_{1}\left(\omega_{n+j}, \omega_{j}\right)(j=m+1, \cdots, n)$, and all other $Q\left(\omega, \omega^{\prime}\right)$ are zero. Thus $Q_{1}$ is the standard skew-form on $W^{1} / W^{0}$ with respect to the basis given in (13.19).

We now compute the induced Hodge filtrations

$$
F_{s}^{k, l}=W^{k} \cap F_{s}^{l} / W^{k-1} \cap F_{s}^{l}
$$

using (13.17) and (13.19). We find that $F^{2,0}=F^{2,1}=W^{2} / W^{1} ; F^{0,0}=\{0\}$ and $F^{0,1}=W^{0}$; and finally

$$
\begin{aligned}
& F_{s}^{1,0}=\left\{\omega_{j}+\sum_{k=m+1}^{n} Z_{j k}(s) \omega_{k}: j=m+1, \cdots, n\right\} ; \\
& F_{s}^{1,1}=\left\{\omega_{j} ; \omega_{n+m+j}: j=m+1, \cdots, n\right\} .
\end{aligned}
$$

From (13.6) and (13.20) it follows that

(i) $W^{0}, W^{2} / W^{1}$ have (trivial) Hodge structures of weights 0 and 2; and

(ii) $W^{1} / W^{0}$ has a Hodge structure of weight 1 which is holomorphic on the whole disc $\Delta$.

Using (i) and (ii) we then verify the Conjecture (9.17).

(c) Completion of the proof of Theorem 13.1. We want to use (13.4), (13.5), and (13.6) to prove $(13.1)^{\prime}$. For this we first need to know something about the period matrix domain $D$ and the Satake compactification $(D / \Gamma) *$ of $D / \Gamma[25]$.

Consider a complex vector space $E$ in which we have a given $Z$-basis (i.e., a given isomorphism $E \cong Z^{2 n} \otimes_{Z} C$ where $\operatorname{dim} E=2 n$ ) and a given nonsingular, skew-symmetric form $Q: E \otimes E \rightarrow C$ which is integral on the $\boldsymbol{Z}$-basis of $E$. For simplicity we will take the principally polarized case, so we assume that $Q$ has the standard matrix (13.4) with respect to a suitable $\boldsymbol{Z}$-basis for $E$.

Let $G(n, E)$ be the Grassman variety of all $n$-planes through the origin in $E$, and let $\check{D}$ be the subvariety of all isotropic $n$-planes $S$; i.e., those $S \in G(n, E)$ which satisfy

$$
Q(S, S)=0 .
$$

The rational, projective variety $\check{D}$ is acted on transitively by the complex, simple Lie group $G_{C} \cong \operatorname{Sp}(2 n, C)$ of all linear transformations $T \in G L(E)$ which preserve $Q$. In $\check{D}$ we take the period matrix domain $D$ (which in this case is the Siegel upper-half-plane $H_{n}$ of genus $n$ ) of all $S \in \check{D}$ which satisfy

$$
i Q(S, \bar{S})>0 \text {. }
$$


The group $G_{R} \cong \operatorname{Sp}(2 n, R)$ of all real linear transformations $T=\bar{T}$ in $G_{C}$ acts transitively on $D$ and preserves the boundary $\partial D=\bar{D}-D$. In fact it is easy to see that $\bar{D}$ is defined by (13.21) and

$$
I Q(S, \bar{S}) \geqq 0 .
$$

Now $G_{R}$ contains both the group of rational points $G_{Q}$ and the arithmetic subgroup $\Gamma \cong \operatorname{Sp}(2 n, \boldsymbol{Z})$ (modular group) of all $T$ which leave invariant the given $Z$-lattice in $E$.

The Satake compactification is obtained by letting $D^{*}$ be the disjoint union of $D$ with the rational boundary components of $D$ in $\check{D}[10],[25]$. Then $\Gamma$ acts on $D^{*}$ and we will have that

$$
\{D / \Gamma\}^{*}=D^{*} / \Gamma,
$$

where a suitable topology has been put on $D^{*}$ so that $D^{*} / \Gamma$ is a separated topological space. In the case at hand the rational boundary components will be in a one-one correspondence with the rational isotropic subspaces $W^{0}$ of $E$ [10]. To each such $W^{0}$ with $\operatorname{dim} W^{0}=m$, we let $F\left(W^{0}\right)$ be the set of all $S \in \check{D}$ with $W^{0} \subset S$ and such that any of the following equivalent conditions is satisfied:

$$
\begin{aligned}
& i Q(S, \bar{S}) \text { has rank } n-m ; \\
& W^{0}=S \cap \bar{S} ; \text { or } \\
& \left(W^{0}\right)^{\perp}=S+\bar{S} .
\end{aligned}
$$

Then $F\left(W^{0}\right)$ is isomorphic to a Siegel-upper-half-plane $H_{n-m}$ of genus $n-m$, the map being that obtained by sending $S \in F\left(W^{0}\right)$ into the $n-m$ plane $S / W^{0}$ of $\left(W^{0}\right)^{\perp} / W^{0}$. Since any two $m$-dimensional rational isotropic subspaces of $E$ are related by a transformation in $\Gamma$ acting on $E$, referring to (13.24) we will have in our case that settheoretically

$$
\{D / \Gamma\}^{*}=\left(H_{n} / \Gamma_{n}\right) \cup\left(H_{n-1} / \Gamma_{n-1}\right) \cup \cdots \cup\left(H_{0} / \Gamma_{0}\right)
$$

where $\Gamma_{p}$ is the modular group acting on $H_{p}$.

At this time, we should point out the connection between the definition of rational boundary components given in $\S 9$ and that just above. In the notation of $\S 9$, the group $N\left(F\left(W^{0}\right)\right)$ is $\left\{T \in G_{R}\right.$ : $\left.T W^{0}=W^{0}\right\}$, and $Z\left(F\left(W^{0}\right)\right)$ is $\left\{T \in N\left(F\left(W^{0}\right)\right): T=I\right.$ on $\left.\left(W^{0}\right)^{\perp} / W^{0}\right\}$. Obviously then $N\left(F\left(W^{0}\right)\right)$ is a maximal parabolic subgroup of $G_{R}$ and is defined over $\boldsymbol{Q}$ and $Z\left(F\left(W^{0}\right)\right)$ is a normal $Q$-subgroup. Observe that $W^{1} / W^{0}$ has a nonsingular skew form

$$
Q_{1}: W^{1} / W^{0} \otimes W^{1} / W^{0} \rightarrow \mathbf{C}
$$


and there is a surjective homomorphism

$$
h: N\left(F\left(W^{0}\right)\right) \rightarrow G L\left(W^{0}, R\right) \times \operatorname{Sp}\left(Q_{1}, R\right)
$$

where $\operatorname{Sp}\left(Q_{1}, R\right)$ is the group of real linear transformations of $W^{1} / W^{0}$ which preserve $Q_{1}$. The kernel of $h$ is the unipotent radical $U\left(F\left(W^{0}\right)\right)$ of $N\left(F\left(W^{0}\right)\right)$, and we also have that $Z\left(F\left(W^{0}\right)\right)$ is the semidirect product $G L\left(W^{0}, R\right) \cdot U\left(F\left(W^{0}\right)\right)$. Note that the P.-L. transformation (13.5) belongs to $U\left(F\left(W^{0}\right)\right)$.

ProOF OF CONVERGENCE IN THE ORDINARY TOPOLOGY. Given a localization $\bar{f}: \bar{Y} \rightarrow \Delta$, we have $E=H^{1}\left(V_{s_{0}}, C\right)$ and may choose bases for $E$ and $F_{s}^{0}$ so that (13.15)-(13.17) are satisfied. The rational boundary component will be $F\left(W^{0}\right)$ where $W^{0}$ is given by (13.18).

Let $s=\exp (2 \pi i t)$ and lift the period mapping to the ordinary upperhalf-plane $H$. Then according to (13.16), (13.17), and (13.19), we can choose a basis $\psi_{1}(t), \cdots, \psi_{n}(t)$ for $F_{t}^{0}$ such that we have:

$$
\begin{aligned}
W^{0} & =\left\{\omega_{n+1}, \cdots, \omega_{n+m}\right\} ; \\
\left(W^{0}\right)^{\perp} & =\left\{\omega_{m+1}, \cdots, \omega_{n}, \omega_{n+1}, \cdots, \omega_{2 n}\right\} ; \\
\psi_{\alpha}(t) & =\frac{1}{t}\left\{\theta_{\alpha}(\exp (2 \pi i t)\}+\sum_{\beta=1}^{m} \Lambda_{\alpha \beta} \omega_{n+\beta} ; \alpha=1, \cdots, m ;\right. \\
\psi_{\rho}(t) & =\omega_{\rho}+\sum_{\sigma=m+1}^{m+n} z_{\rho \sigma}(\exp (2 \pi i t)) \omega_{n+\sigma} ; \rho=m+1, \cdots, n ;
\end{aligned}
$$

where $\theta_{\alpha}(\exp (2 \pi i t))$ and $z_{\rho \sigma}(\exp (2 \pi i t))$ are holomorphic on the whole disc $\operatorname{Im} t>0$. From (13.27) it follows that

$$
\lim _{\operatorname{Im} t \rightarrow+\infty} F_{t}{ }^{0}=S \in F\left(W^{0}\right),
$$

where the limit is taken in the ordinary topology of $D \subset \check{D}$ (cf. (4.3)).

Proof of convergence in the Satake topology. For this we will set $s=\exp (2 \pi i t)$ as above, and will change the ordering of our basis of $H_{1}\left(V_{s_{0}}, Q\right)$ to $\delta_{m+1}, \cdots, \delta_{n} ; \delta_{1}, \cdots, \delta_{m} ; \delta_{n+m+1}, \cdots, \delta_{2 n}$; $\delta_{n+1}, \cdots, \delta_{n+m}$ so that $(13.6)$ becomes

$$
Z=\imath\left(\begin{array}{ll}
0 & 0 \\
0 & \Lambda
\end{array}\right)+\left(\begin{array}{cc}
Z_{11} & Z_{12} \\
{ }^{t} Z_{12} & Z_{22}
\end{array}\right), \quad \Lambda={ }^{t} \Lambda>0,
$$

where the $Z_{i j}$ are holomorphic functions of $s=\exp (2 \pi i t)$ at $s=0$.

Now we shall describe explicitly a fundamental set or Siegel domain [10] for modular group acting on the Siegel-upper-half-plane $H_{n}$. For this we write $Z \in H_{n}$ as $Z=X+i Y$ and

$$
Y={ }^{t} W D W
$$


where

$$
W=\left(\begin{array}{ccc}
1 & \cdots & w_{1 n} \\
\vdots & \ddots & \vdots \\
0 & \cdots & 1
\end{array}\right)
$$

is upper triangular and

$$
D=\left(\begin{array}{ccc}
d_{1} & \cdots & 0 \\
\vdots & \ddots & \vdots \\
0 & \cdots & \vdots \\
0 & \cdots & d_{n}
\end{array}\right)
$$

is diagonal with positive entries. Choose a large positive real number $u$. Then a fundamental set $\Im_{n}$ for $H_{n}$ is given by the $Z=X+i Y$ which satisfy

$$
\begin{aligned}
& \left|x_{i j}\right|<u, \\
& \left|w_{i j}\right|<u, \\
& 1 \leqq d_{1}, \quad d_{i} \leqq u d_{i+1} \quad \text { for } i=1, \cdots, n-1 .
\end{aligned}
$$

Write $Z^{\prime}=Z_{11}(0) \in H_{n-m}$ as $Z^{\prime}=X^{\prime}+i Y^{\prime}$. By a transformation in the modular group operating on $W^{1} / W^{0}$ we may assume that $Z^{\prime}$ satisfies the conditions (13.31) for $\mathcal{F}_{n-m}$ in $H_{n-m}$. By possibly making $u$ larger, the same will be true for $Z_{11}(s)$ when $|s|<\epsilon$.

Now write $\Lambda={ }^{t} V \Delta V$ where

$$
V=\left(\begin{array}{ccc}
1 & \cdots & v_{1 m} \\
\vdots & \ddots & \vdots \\
0 & \cdots & 1
\end{array}\right)
$$

is upper triangular and

$$
\Delta=\left(\begin{array}{ccc}
\delta_{1} & \cdots & 0 \\
\vdots & \ddots & \vdots \\
\dot{0} & \cdots & \delta_{m}
\end{array}\right)
$$

is diagonal. By renumbering our rational basis for $W^{0}$, we may assume that $0<\delta_{1} \leqq \cdots \leqq \delta_{m}$. Let $t=\xi+i \eta$. We may restrict ourselves to $|\xi| \leqq v, \eta>v$. Then $t \Lambda=\xi \Lambda+i\left({ }^{t} V \eta \Delta V\right)$. It follows that we may assume $\left|x_{i j}(t)\right| \leqq u$ for all such $t$. Also we have

$$
Y=\left(\begin{array}{cc}
0 & 0 \\
0 & { }^{t} V \eta \Delta V
\end{array}\right)+\left(\begin{array}{cc}
Y_{11} & Y_{12} \\
t Y_{12} & Y_{22}
\end{array}\right), \quad \text { and }
$$




$$
\begin{aligned}
Y & =\left(\begin{array}{cc}
{ }^{t} W_{11} & 0 \\
{ }^{t} W_{12} & { }^{t} W_{22}
\end{array}\right)\left(\begin{array}{cc}
D_{1} & 0 \\
0 & D_{2}
\end{array}\right)\left(\begin{array}{cc}
W_{11} & W_{12} \\
0 & W_{22}
\end{array}\right) \\
& =\left(\begin{array}{cc}
{ }^{t} W_{11} D_{1} W_{11} & { }^{t} W_{11} D_{1} W_{12} \\
{ }^{t} W_{12} D_{1} W_{11} & { }^{t} W_{12} D_{1} W_{12}+{ }^{t} W_{22} D_{2} W_{22}
\end{array}\right) .
\end{aligned}
$$

Comparing (13.32) and (13.33) we see that $Z(t) \in \mathcal{F}_{n}$ for all $t$ if we agree to take $u$ large enough. In other words, we have a holomorphic mapping

$$
\phi: H \rightarrow D \subset \check{D}
$$

from the usual upper half plane $\{t=\xi+i \eta: \eta>0\}$ to the Siegelgeneralized-upper-half-plane $H_{n}=D$ which satisfies

(i) $\phi(t+1)=T \phi(t)$ where $T \in \Gamma$, which is the modular group acting on $D$; and

(ii) $\phi$ maps the strip $|\xi|\langle v, \eta\rangle v$ into a Siegel domain $\mathfrak{F} \subset D$;

(iii) $\lim _{\eta \rightarrow+\infty} \phi(\xi+i \eta)$ is a point in a Siegel domain $\mathfrak{F}_{n-m}$ on an $H_{n-m}$ embedded in $\partial D$ as a rational boundary component, and where the limit is in the usual topology of $\breve{D}$. It follows from (i)-(iv) that we have convergence in the sense of Satake.

14. Periods of certain algebraic surfaces acquiring singularities. My purpose in this section is to discuss and partially verify the Conjectures 9.2, 9.5, and 9.17 in a substantial example for $q=2$ (periods of 2 -forms). The term "substantial example" is based on the following heuristic considerations: Let $V_{s}$ be an analytic family of algebraic surfaces in some $P_{N}$ which is parametrized by the unit $s$-disc $\Delta$ in $C$, and where $V_{s}$ is nonsingular for $s \neq 0$ and $V_{0}$ is irreducible. By resolution of singularities, $V_{0}$ will have a desingularization $\tilde{V}_{0}$ in some $P_{N^{\prime}}$.

Now generic projection of $P_{N}$ into $P_{3}$ gives a family of surfaces $W_{s}$, $W_{s}$ being the projection of $V_{s}$, which have only ordinary singularities (cf. Kodaira [21], Lefschetz [7], and (14.1) below). A reasonable geometric assumption is that the projection $W_{0}$ of $V_{0}$ still has only ordinary singular points, but has acquired additional new ones from those imposed by $W_{s}$ as $s$ tends to zero (cf. Mumford's theorem quoted in the proof of Proposition 15.6 below).

Since we are interested in periods of 2 -forms, another reasonable hypothesis is that the surfaces $V_{s}$ are simply-connected so that in particular the irregularity $h^{1,0}\left(V_{s}\right)=0(s \neq 0)$.

To be precise, we assume given a local situation $f: \bar{Y} \rightarrow \Delta$ as in $\S 8(\mathrm{a})$, and where $V_{s}(s \neq 0)$ is a nonsingular, simply-connected algebraic surface. We will assume that $V_{0}$ is irreducible, and that we can 
find local holomorphic coordinates $u, v, w$ on $\bar{Y}$ such that $V_{0}$ has one of the following three local equations (definition of ordinary singularities):

$$
u v=0 \quad \text { (double points) }
$$

$(14.1)_{t} \quad u v w=0 \quad$ (triple points)

$$
u v^{2}=w^{2} \text { (cuspidal points) }
$$
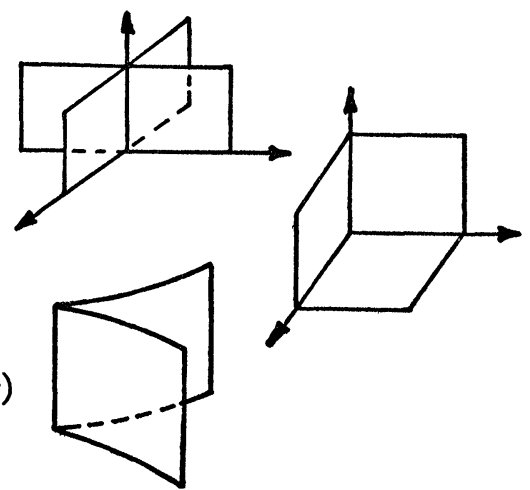

We will also make use of a projective embedding $\bar{Y} \hookrightarrow P_{N}$ and generic projection $P_{N} \rightarrow P_{3}$, so that in the diagram

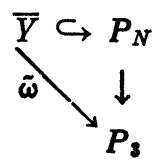

the projected surfaces $W_{s}=\tilde{\omega}\left(V_{s}\right)$ are irreducible surfaces with ordinary singularities given by the affine equation

$$
f(x, y, z ; s)=0,
$$

where $x, y, z$ are coordinates in $C^{3}$ and where $f(x, y, z ; s)$ is an irreducible polynomial of degree $n$ whose coefficients are holomorphic functions of $s$.

Referring to (14.1), the singular curve $C$ on $V_{0}$ has the respective local equations

$$
\text { (14.4) } \begin{aligned}
u & =0, \quad v=0 \\
u & =0, \quad v=0 \quad \text { or } \quad u=0, \quad w=0 \quad \text { or } \quad v=0, \quad w=0 \\
v & =0, \quad w=0
\end{aligned}
$$

Thus the only singularities of $C$ are triple points at those of $V_{0}$. The normalization $\tilde{V}_{0}$ of $V_{0}$ is also a desingularization, and we have a diagram 


$$
\begin{aligned}
& \nabla_{0} \supset C \\
& \pi \downarrow \downarrow \pi \\
& V_{0} \supset C,
\end{aligned}
$$

where $\tilde{C}$ is the inverse image of $C$ in $\tilde{V}_{0}$. The normalization locally around double points and triple points of $V_{0}$ is clear, and the normalization around the cuspidal point is given by (cf. (14.1) $)_{\mathrm{o}}$ )

$$
(\xi, \eta) \rightarrow\left(\xi^{2}, \eta, \xi \eta\right) .
$$

Referring to (14.4) and (14.6) we see that:

The curve $\tilde{C}$ has only ordinary double points, and the projection $\pi: \widetilde{C} \rightarrow C$ is a 2 -sheeted covering with simple ramification of order two at each cuspidal point, and with three double points of $\tilde{C}$ lying over each triple point of $C$.

We will assume that $\tilde{C}$ is irreducible. This is the general and more interesting case, and the al ternatives can be done by similar methods. The number of cuspidal points on $C$ is then an even number $2 \sigma$.

Let $C^{\prime}$ and $\tilde{C}^{\prime}$ be the respective normalizations of $C$ and $\tilde{C}$. We have a diagram

$$
\begin{aligned}
\tilde{C}^{\prime} & \rightarrow \tilde{C} \\
\pi \downarrow & \\
C^{\prime} \rightarrow & \downarrow \pi
\end{aligned}
$$

and the Riemann-Hurwitz formula gives the relation

$$
h^{1,0}\left(C^{\prime}\right)=2 h^{1,0}\left(C^{\prime}\right)-1+\sigma .
$$

In fact, we have

(14.9). Proposition. There is an involution $j: \widetilde{C}^{\prime} \rightarrow \widetilde{C}^{\prime}$ with $\widetilde{C}^{\prime} /(j)$ $=C^{\prime}$ in (14.7). Letiing $h^{1,0}(j,+)$ and $h^{1,0}(j,-)$ be the respective dimensions of the +1 and -1 eigenspaces of $j$ acting on $H^{0}\left(\tilde{C}^{\prime}, \Omega^{1}\right)$, we have $h^{1,0}(j,+)=h^{1,0}\left(C^{\prime}\right)$ and

$$
h^{1,0}(j,-)=h^{1,0}\left(C^{\prime}\right)-1+\sigma .
$$

Having formulated our example and established the necessary notations, we will divide the remainder of this paragraph into four sections. The first deals with the behaviour of the holomorphic 2forms on $V_{s}$ as $s \rightarrow 0$; the second section discusses the topology of $V_{s}$ and $V_{0}$, and the P.-L. transformation $T$ around $s=0$; in the third section we will put everything together to find out formulae for the periods; and in the fourth section we will draw some conclusions. 
(a) Behaviour of differentials. We will use the notations $h^{1,0}(C)$ $=h^{1,0}\left(C^{\prime}\right), h^{1,0}(\tilde{C})=h^{1,0}\left(\widetilde{C}^{\prime}\right)$, and $h^{2,0}\left(V_{0}\right)=h^{2,0}\left(\tilde{V}_{0}\right)$. Our purpose is to describe the differentials in $H^{0}\left(V_{s}, \Omega_{V_{s}}^{2}\right)$ as $s$ tends to zero (written $s \rightarrow 0$ ). In particular, we will outline a proof of

(14.10). Proposition. The following relation holds

$$
h^{2,0}\left(V_{s}\right)=h^{2,0}\left(V_{0}\right)+h^{1,0}(\widetilde{C})-h^{1,0}(C)+\tau,
$$

where $\tau$ is the number of triple points on $C$.

REMARK. For curves the analogous formula is (15.8) below.

Proof. For the proof we will use the notion of a Poincare residue. Let $M$ be a complex manifold of dimension $m, N \subset M$ an analytic hypersurface, and $\omega$ a meromorphic $m$-form on $M$ with a first order pole along each of the irreducible components of $N$. The Poincaré residue $R(\omega)$ will be a meromorphic $(m-1)$ form on each irreducible component of $N$. For our purposes it will suffice to say what the differential $f^{*} R(\omega)$ is whenever $f: N^{\prime} \rightarrow N$ is a holomorphic mapping of a connected, $(m-1)$-dimensional complex manifold $N^{\prime}$ into $M$ such that the differential of $f$ has maximal rank at some point. With these assumptions, $f^{*} R(\omega)$ will be determined if we say what $R(\omega)$ is at a simple point of $N$. For this we can choose local holomorphic coordinates $z_{1}, \cdots, z_{m}$ on $M$ such that $N$ is given by $z_{m}=0$, and then $\omega$ has an expression $\omega=\left(F(z) d z_{1} \wedge \cdots \wedge d z_{m}\right) / z_{m}$ where $F(z)$ is holomorphic. We define

$$
R(\omega)=F\left(z_{1}, \cdots, z_{m-1}, 0\right) d z_{1} \wedge \cdots \wedge d z_{m-1} .
$$

With this preliminary about residues understood, we recall [7] that the holomorphic 2-forms on $V_{s}$ are given on $W_{s}$ by (cf. (14.3))

$$
\phi(s)=P(x, y, z ; s) d x \wedge d y / \partial f / \partial z(x, y, z ; s)
$$

where $P(x, y, z ; s)$ is a polynomial of degree $n-4$ whose coefficients are holomorphic functions of $s$ and which vanishes on the double curve $C_{s}$ of $W_{s}$ (adjoint conditions). Comparing (14.11) and (14.12) we see that $\phi(s)=R \omega(s)$ where

$$
\omega(s)=(P(x, y, z ; s) d x \wedge d y \wedge d z) / f(x, y, z ; s)
$$

is a meromorphic 3 -form on $P_{3}$ with a first order pole along $W_{8}$. As $s \rightarrow 0$ we will have $\omega(s) \rightarrow \omega(0)$ where

$$
\omega(0)=(P(x, y, z ; 0) d x \wedge d y \wedge d z) / f(x, y, z ; 0)
$$

and where $P(x, y, z ; 0)$ need not vanish on the new double curve $\tilde{\omega}(C)$ 
of $V_{0}$. Using (14.1) and (14.6) we see that the Poincare residue $\phi=\phi(0)$ of $\omega(0)$ is given locally by
$(14.13)_{\mathrm{d}}$
$\phi=F\{d v d w / v\}$,
$\phi=F\{d v d w / v w\}$,$$
\phi=F\{d u d v / w\}=F\{d \xi d \eta / \eta\},
$$

where $F$ is a holomorphic function. Thus, in all cases, $\phi$ is a meromorphic 2 -form on $\tilde{V}_{0}$ with a pole of order one along $\tilde{C}$ (cf. (14.5)). The Poincare residue $\psi=R \phi$ of $\phi$ along $\widetilde{C}$ is then a meromorphic 1form on the normalization $\widetilde{C}^{\prime}$ of $\widetilde{C}$ (cf. (14.7)) with logarithmic singularities (first order poles) at the $6 \tau$ points on $\tilde{C}^{\prime}$ lying over the $\tau$ triple points on $C$, and which satisfies

$$
j^{*} \psi=-\psi
$$

where $j: \tilde{C}^{\prime} \rightarrow \tilde{C}^{\prime}$ is the canonical involution given by (14.7). Note that the usual relation

$$
\sum_{x \in \tilde{C}^{\prime}} \operatorname{Res}_{x}(\psi)=0
$$

is in our situation a consequence of (14.14). From (14.13), (14.14), and (14.15) it follows that

$$
h^{2,0}\left(V_{s}\right) \leqq h^{2,0}\left(V_{0}\right)+h^{1,0}(\tilde{C})-h^{1,0}(C)+\tau .
$$

The equality in (14.16) now results from the 'classical' theory of algebraic surfaces (cf. Zariski, Algebraic surfaces (Chelsea), p. 64).

(14.17). Remark. The above discussion tells us that we can find a basis

$$
\begin{array}{ll}
\phi_{1}(s), \cdots, \phi_{h_{0}}(s) & h_{0}=h^{2,0}\left(V_{0}\right) \\
\psi_{1}(s), \cdots, \psi_{\tilde{p}-p}(s) & \tilde{p}=h^{1,0}(C), p=h^{1,0}(C) \\
\eta_{1}(s), \cdots, \eta_{\tau}(s) & \tau=\text { number of triple points }
\end{array}
$$

for $H^{0}\left(V_{s}, \Omega_{V_{s}}^{2}\right)(s \neq 0)$ which varies holomorphically with $s \in \Delta$ and which has the following properties:

(i) the $\phi_{a}(s), \psi_{i}(s)$, and $\eta_{m}(s)$ are Poincaré residues on $V_{0}$ of the form given by (14.12);

(ii) the $\phi_{a}=\phi_{a}(0)$ are a basis for the holomorphic 2 -forms on $\tilde{V}_{0}$ (i.e. the $P_{a}(x, y, z ; 0)$ vanish on $C$ and no nontrivial linear combination of the $P_{a}(x, y, z ; 0)$ is zero);

(iii) the $\psi_{i}=\psi_{i}(0)$ are a basis for the meromorphic 2-forms on $\tilde{V}_{0}$ whose Poincaré residues $R \psi_{i}$ on $\tilde{C}$ induce holomorphic 1 -forms on 
$\tilde{C}^{\prime}$ (i.e. the $P_{i}(x, y, z ; 0)$ vanish at the triple points of $C$ and no nontrivial linear combination of the $P_{i}(x, y, z ; 0)$ vanishes on $\left.C\right)$; and

(iv) the Poincaré residues $R \eta_{m}\left(\eta_{m}=\eta_{m}(0)\right)$ induce meromorphic 1 -forms on $\tilde{C}^{\prime}$ which are linearly independent modulo holomorphic 1 -forms there (i.e. no nontrival linear combination of the $P_{m}(x, y, z ; 0)$ vanishes at a triple point of $C$ ).

(14.18). Remark. According to the Poincare complete reducibility theorem, we can find a basis $\theta_{1}, \cdots, \theta_{\tilde{p}}\left(\tilde{p}=h^{1,0}(\tilde{C})\right)$ for $H^{0}\left(\tilde{C}^{\prime}, \Omega_{\tilde{C}^{1}}\right)$ and a basis $\gamma_{1}, \cdots, \gamma_{2}$ for $H_{1}\left(\tilde{C}^{\prime}, Q\right)$ with the following properties:

(i) $j^{*} \theta_{k}=-\theta_{k}$ for $k=1, \cdots, \tilde{p}-p\left(p=h^{1,0}(C)\right)$ where $j: \tilde{C}^{\prime} \rightarrow \tilde{C}^{\prime}$ is the involution (14.7). In fact, we may assume that

$$
\theta_{k}=R \psi_{k} \quad(k=1, \cdots, \tilde{p}-p)
$$

in the notation of Remark (14.17);

(ii) the intersection matrix of $\gamma_{1}, \cdots, \gamma_{2 \tilde{p}}$ is

$$
\widetilde{Q}=\left(\begin{array}{cc|cc}
0 & I_{\tilde{p}-p} & 0 & 0 \\
-I_{\tilde{p}-p} & 0 & 0 & 0 \\
\hline 0 & 0 & 0 & I_{p} \\
0 & 0 & -I_{p} & 0
\end{array}\right) ;
$$

and

(iii) the period matrix $\left(\int_{\gamma_{p}} \theta_{k}\right)$ splits as a direct sum

$$
\tilde{\Omega}=\left(\begin{array}{cccc}
I_{\tilde{p}-p} & 0 & Z^{\prime} & 0 \\
0 & I_{p} & 0 & Z^{\prime \prime}
\end{array}\right)
$$

where $Z^{\prime}, Z^{\prime \prime}$ are both in appropriate Siegel-upper-half-planes (cf. $\$ 13$ above).

We will use below the notation

$$
\Omega^{\prime}=\left(I_{\tilde{p}-p}, Z^{\prime}\right)
$$

for the period matrix

$$
\left(\int_{\gamma_{\alpha}} R \psi_{k}\right) \quad(1 \leqq k \leqq \tilde{p}-p ; 1 \leqq \alpha \leqq 2 \tilde{p}-2 p) .
$$

(b) Topology and the P.-L. transformation. In this section we will extract the results from [14] necessary to give a picture of the topology of $V_{s}$ near $V_{0}$ (cf. also $\$ 15$ below). For this we picture $V_{s}(s \neq 0)$ as a generalized fibre space

$$
h: V_{s} \rightarrow V_{0}
$$


lying over $V_{0}$ (cf. $\$ 2$ in [14] and $\$ 15$ (b) below). The fibres of $h$ have the following description:

(i) For $x \in V_{0}-C, h^{-1}(x)$ is just a single point.

(ii) For $x \in C-\{$ triple and cuspidal points $\}, h^{-1}(x)$ is topologically $C^{*}$ :

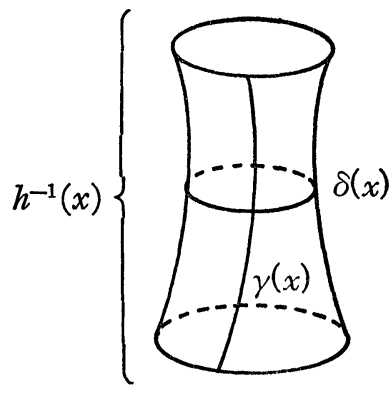

Figure 1

Thus $h^{-1}(x)$ has the homotopy type of an oriented circle $\delta(x)$, which we will call the vanishing cycle over $x$. The Poincaré-Lefschetz dual $\gamma(x)$ of $\delta(x)$ in $h^{-1}(x)$ will be termed the transverse cycle over $x$.

(iii) For $x$ a triple point on $C, h^{-1}(x)$ is topologically $C^{*} \times C^{*}$ and thus has the homotopy type of an oriented 2-torus $\Delta(x)$, which we will call the vanishing cycle associated to the triple point $x$.

(iv) For $x$ a cuspidal point, the fibre $h^{-1}(x)$ is somewhat complicated and all we shall need are the following two facts: the fibre $h^{-1}(x)$ is topologically a manifold with boundary, the intersection pairing on $H_{2}\left(h^{-1}(x), Q\right)$ is nonsingular, and the local P.-L. transformation $T$ is of finite order 6 on $H_{2}\left(h^{-1}(x), Q\right)$; and

(14.20) as $y \in C$ turns around a cuspidal point $x$, the vanishing cycle $\delta(y)$ reverses orientation (cf. Figure 1 ).

To describe the topology of $V_{8}$ near $V_{0}$, we replace $T$ by $T^{6}$ so as to get rid of the finite order material around the cuspidal point thus making $T$ unipotent, and we also assume that the intersection number $(\tilde{C} \cdot \tilde{C}) \tilde{V}_{0}>0$. This is the general case and the alternative can be treated by a similar argument.

We divide the homology $H_{2}\left(V_{s}, Q\right)$ into three groups of cycles.

(C-1). Cycles associated to triple points. A basis (over $Q$ ) for these cycles will be labelled ( $\tau=$ number of triple points)

$$
\Delta_{1}, \cdots, \Delta_{\tau} ; \quad \Phi_{1}, \cdots, \Phi_{\tau} ; \quad \Psi_{1}, \cdots, \Psi_{\tau}
$$

and they have the following properties and interpretations: 
(i) the intersection matrix for the cycles in (14.21) is an orthogonal direct sum of $\tau 3 \times 3$ matrices, each of which is

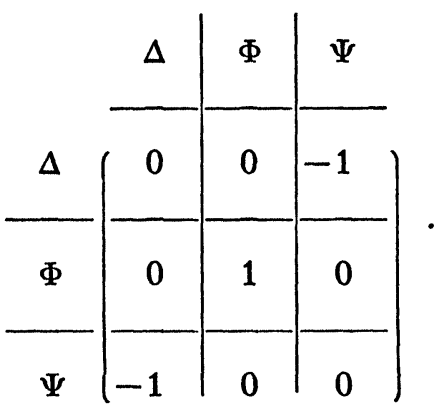

This matrix is equivalent over $R$ to

$$
\left(\begin{array}{rrr}
1 & 0 & 0 \\
0 & 1 & 0 \\
0 & 0 & -1
\end{array}\right),
$$

and so the cycles in (14.21) contribute $2 \tau$ positive and $\tau$ negative eigenvalues to the intersection matrix $Q$ for $V_{8}$.

(ii) The P.-L. transformation on the cycles in (14.21) is given by

$$
\begin{aligned}
& T \Delta=\Delta \\
& T \Phi=\Phi+\Delta \\
& T \Psi=\Psi+\Phi+\frac{1}{2} \Delta
\end{aligned}
$$

and thus $T$ on these cycles is a direct sum of $\tau$ copies of the standard Jordan matrix

$$
\left(\begin{array}{lll}
1 & 1 & \frac{1}{2} \\
0 & 1 & 1 \\
0 & 0 & 1
\end{array}\right)=\exp \left(\begin{array}{lll}
0 & 1 & 0 \\
0 & 0 & 1 \\
0 & 0 & 0
\end{array}\right) .
$$

(iii) The $\Delta_{m}$ are the vanishing cycles associated to the $\tau$ triple points of $V_{0}$ along $C$. These cycles are independent in $H_{2}\left(V_{s}, Q\right)$ since $\pi_{1}\left(V_{s}\right)=0$.

(iv) To describe the $\Phi_{m}$ and $\Psi_{m}$, we choose for each triple point $x$ three cuspidal points $y_{1}, y_{2}, y_{3}$ and, on each of the three branches of $C$ passing through $x$, we draw a closed curve from $x$ around one of the $y_{j}$ 's. (cf. $\left.(14.1)_{t}\right)$ 


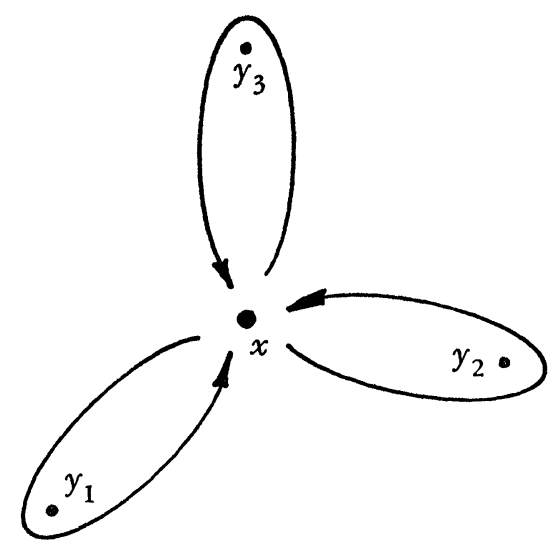

FIGURE 2

(v) To define $\Phi(x)$ (which is the particular one of the $\Phi_{m}$ associated to the triple point $x$ ), we will describe $\Phi(x)$ up to multiplicities and a linear combination of $\Delta_{1}, \cdots, \Delta_{m}$. The locus of the vanishing cycle $\delta(y)$ as $y$ traverses one of the paths in Fig. 2 is a 2-chain whose boundary is twice the circle $\lim _{y \rightarrow x} \delta(y)$; this circle is sitting in the 2-torus $\Delta(x)$ (cf. (14.20)). A linear combination of these three 2chains will then give a 2-chain whose boundary is also the boundary of a 2-chain in $\Delta(x)$. Adding on minus this 2-chain from $\Delta(x)$, we obtain the cycle $\Phi(x)$.

(vi) We will similarly describe $\Psi(x)$ up to multiplicities and linear combinations of $\Phi_{1}, \cdots, \Phi_{m}$ and $\Delta_{1}, \cdots, \Delta_{r}$. This description will also only describe $\Phi(x) \cap h^{-1}(C)$, and to do this we take the locus of the transverse cycle $\gamma(y)$ as $y$ describes the paths in Figure 2, and where it is agreed to add in the limiting position $\lim _{y \rightarrow x} \gamma(y)$.

From these descriptions it is not hard to see that the cycles in (14.21) as given by (iii)-(vi) may be normalized so that (14.22) and (14.23) hold true.

(C-2). Cycles over the double curve. A basis over $Q$ for these cycles will be labelled

$$
\begin{aligned}
& \Theta_{1}, \cdots, \Theta_{2 \tilde{p}-2 p} \\
& \Gamma_{1}, \cdots, \Gamma_{2 \tilde{p}-2 p}
\end{aligned}
$$

where $\tilde{p}=h^{1,0}(\tilde{C}), p=h^{1,0}(C)$ and where we have the relation

$$
2 \tilde{p}-2 p=2 p+2 \sigma-2
$$

by (14.8). The cycles in (14.24) have the following properties and 
interpretations:

(i) the intersection matrix is

$$
\left(\begin{array}{cccl}
0 & 0 & 0 & I_{\dot{p}-p} \\
0 & 0 & -I_{\tilde{p}-p} & 0 \\
0 & -I_{\tilde{p}-p} & 0 & 0 \\
I_{\tilde{p}-p} & 0 & 0 & 0
\end{array}\right) .
$$

This matrix is equivalent to

$$
\left(\begin{array}{cc}
I_{2 \tilde{p}-2 p} & 0 \\
0 & -I_{2 \tilde{p}-2 p}
\end{array}\right),
$$

and so the cycles in (14.25) contribute $2 \tilde{p}-2 p$ positive and $2 \tilde{p}-2 p$ negative eigenvalues to $Q$.

(ii) The P.-L. transformation on the cycles in (14.25) is given by

$$
\begin{aligned}
& T \Theta_{\rho}=\Theta_{\rho} \\
& T \Gamma_{\rho}=\Gamma_{\rho}+\Theta_{\rho} .
\end{aligned}
$$

(iii) To describe the $\Theta^{\prime}$ 's we let $C^{*}=C-\{$ triple and cuspidal points $\}, \widetilde{C}^{*}=\widetilde{C}-\{$ double points and branch points of $\pi: \widetilde{C} \rightarrow C\}$, and $\pi: \widetilde{C}^{*} \rightarrow C^{*}$ the projection (cf. $(14.5)$ ). Let $H_{1}(\widetilde{C},-)$ be the -1 eigenspace of the involution $j_{*}: H_{1}\left(\widetilde{C}^{\prime}, Q\right) \rightarrow H_{1}\left(\tilde{C}^{\prime}, Q\right)$ so that the rank of $H_{1}(\tilde{C},-)$ is $2 p-2 \sigma-2$ (cf. (14.9) and (14.25)). Choose cycles $\tilde{\theta}_{1}$, $\cdots, \tilde{\theta}_{2 p+2 \sigma-2}$ in $H_{1}\left(\widetilde{C}^{*}, Q\right)$ which give a basis for $H_{1}(\widetilde{C},-)$ and whose intersection matrix is

$$
\left(\begin{array}{cc}
0 & I_{\tilde{p}-p} \\
-I_{\tilde{p}-p} & 0
\end{array}\right),
$$

as in Remark 14.18. Let $\theta_{\rho}=\pi_{*} \tilde{\theta}_{\rho}$ and $\Theta_{\rho}$ be the locus of the vanishing cycle $\delta(x)$ as $x$ traverses $\theta_{\rho}$.

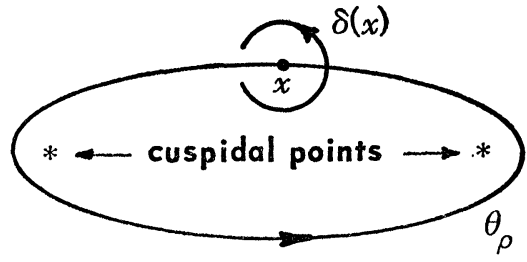

Figure 3

(iv) The $\Gamma_{\rho}$ are defined in $h^{-1}(C)$ as the locus of the transverse 
cycle $\gamma(x)$ as $x$ traverses the dual cycle $\check{\theta}_{\rho}$ to $\theta_{\rho}$ (i.e. $\check{\theta}_{\rho}=\theta_{\tilde{p}-p+\rho}$ if $\rho \leqq \tilde{p}-p$ and $\tilde{\theta}_{\rho}=-\theta_{\rho-\tilde{p}+p}$ if $\left.\rho>\tilde{p}-p\right)$.

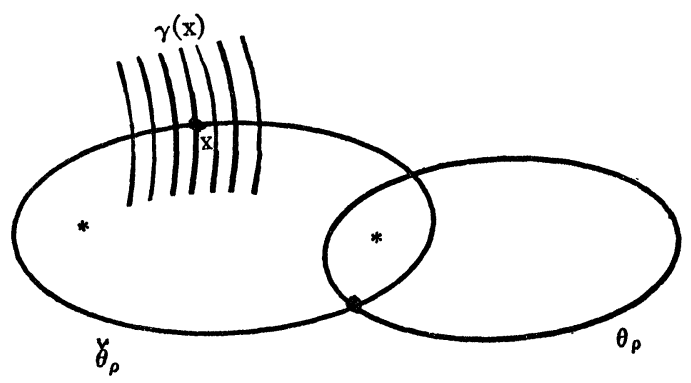

FigURE 4

(C-3). Cycles away from the singularities. A basis for these cycles will be

$$
\Sigma_{1}, \cdots, \Sigma_{b},
$$

and they are described as the cycles in $H_{2}\left(V_{s}-h^{-1}(C), Q\right)$ which inject into $H_{2}\left(V_{s}, Q\right)$. Since we are assuming $\tilde{C}$ to be the polarizing class on $\tilde{V}_{0}$, we see that

$$
\left(Q \cdot \Sigma_{1}\right) \oplus \cdots \oplus\left(Q \cdot \Sigma_{b}\right) \sim H_{2}\left(\tilde{V}_{0}, Q\right)_{0},
$$

where $H_{2}\left(\tilde{V}_{0}, Q\right)_{0}$ is the primitive part [6] of $H_{2}\left(\tilde{V}_{0}, Q\right)$. The cycles $\Sigma_{j}$ are all invariant and have intersection matrix $Q_{0}=\left(\Sigma_{j} \cdot \Sigma_{k}\right)$.

Combining this remark with $(14.26)^{\prime}$ and $(14.22)^{\prime}$ we have that: The number of positive eigenvalues in the intersection matrix $Q$ for $V_{s}$ is equal to

$$
2 \tau+(2 \tilde{p}-2 p)+\left(\# \text { positive eigenvalues in } Q_{0}\right) \text {. }
$$

Using this formula together with the Hodge index theorem [6] we obtain another proof of (14.10).

Finally we have from (14.23) and (14.27) the expression relating the P.-L. transformation to the geometric genera of $V_{8}$ and $\widetilde{V}_{0}$ :

$$
\frac{1}{2} \operatorname{dim}\left\{(T-I) H_{2}\left(V_{s}, Q\right)\right\}=h^{2,0}\left(V_{s}\right)-h^{2,0}\left(V_{0}\right) .
$$

The analogous formula for curves is (15.10) below.

(c) Asymptotic formulae for periods. We want to give the formulae for the intersection matrix, P.-L. transformation, and period matrix of $V_{s}$ as $s$ tends toward zero. In order to condense the notation, these matrices will be given in "block form." For example the matrix 


$$
\begin{array}{ccc}
\Delta & \Phi & \Psi \\
{ }_{\psi}\left(\begin{array}{ccc}
\cdot & \cdot & \cdot \\
\cdot & \cdot & \cdot
\end{array}\right)
\end{array}
$$

will be the abbreviation for the matrix

$$
\Delta_{1} \cdots \Delta_{\tau} \quad \Phi_{1} \cdots \Phi_{\tau} \quad \Psi_{1} \cdots \Psi_{\tau}
$$

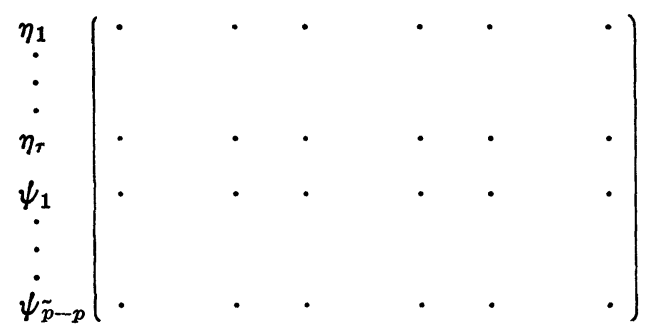

whose entries (in this case) are the periods $\int_{\Delta_{m}} \eta_{j}, \int_{\Delta_{m}} \psi_{j}, \cdots$ of certain of the holomorphic 2-forms on $V_{s}$ (cf. (14.17) and (14.22)). As another

\begin{tabular}{|c|c|c|c|c|c|c|}
\hline & $\Delta$ & $\Phi$ & $\Psi$ & $\Theta$ & $\Gamma$ & $\Sigma$ \\
\hline 4 & 0 & 0 & $-I_{\tau}$ & & & \\
\hline$\Phi$ & 0 & $I_{\tau}$ & 0 & & & \\
\hline$\Psi$ & $-I_{\tau}$ & 0 & 0 & & & \\
\hline$\Theta$ & & & & 0 & $J$ & \\
\hline$\Gamma$ & & & & $-J$ & 0 & \\
\hline & & & & & & \\
\hline & & & & & & $Q_{0}$ \\
\hline
\end{tabular}
example, the full intersection matrix $Q$ on $\mathrm{H}_{2}\left(V_{s}, Q\right)_{0}$ is written as

where $J$ is the matrix

$$
\left(\begin{array}{cc}
0 & I_{\tilde{p}-p} \\
-I_{\tilde{p}-p} & 0
\end{array}\right)
$$

(cf. (14.26)) and where the blank spaces in (14.32) mean zero.

Before giving the period matrix we will make two normalizations. I will take the basis $\phi, \eta, \psi$ for $H^{0}\left(V_{s}, \Omega_{V_{s}}^{2}\right)$ given in Remark 14.17, and the basis

$$
\Psi, \Phi, \Delta ; \Gamma, \Theta ; \Sigma
$$


for $H_{2}\left(V_{s}, Q\right)_{0}$ as given by (14.21), (14.24), and (14.29); and whose intersection matrix is (14.32). According to (iv) in Remark 14.17 we may assume that

$$
\int_{\Delta_{m}} \eta_{k} \sim \delta_{m}^{k} \quad(1 \leqq k, m \leqq \tau),
$$

where the notation " $a \sim b$ " means that $\lim _{s \rightarrow 0}\left|s^{-\lambda}\right||a-b|=0$ for any $\lambda$ with $0 \leqq \lambda<1$ (thus e.g. $s \log s \sim 0$ ). The second normalization is that, according to (iii) in Remark 14.17 and (14.19) in Remark 14.18, we may assume

$$
\left(\int_{\Theta_{\rho}} \psi_{i}\right) \sim \Omega^{\prime} \quad(1 \leqq i \leqq \tilde{p}-p ; 1 \leqq \rho \leqq 2 \tilde{p}-2 p),
$$

where $\Omega^{\prime}=\left(I_{\tilde{p}-p}, Z^{\prime}\right)$ in $(14.19)^{\prime}$.

The entries in the period matrix will be given only in the sense of the notation " " explained above, so that in effect we are only giving it asymptotically. Also the following notations will be used: $l=\log s / 2 \pi i, H_{j}$ will be a holomorphic matrix for any index $j$, and finally we will write

$$
\left(\int_{\Sigma_{j}} \phi_{a}(s)\right) \sim \Omega_{0}
$$

where $\Omega_{0}$ is the period matrix for the holomorphic 2 -forms on the normalization $\tilde{V}_{0}$ of $V_{0}$ (cf. (ii) in Remark 14.17 and (14.30)).

(14.36). Proposition. With the conventions and notations just given, the period matrix $\Omega(s)$ for $V_{s},|s|<\epsilon$, is in block form

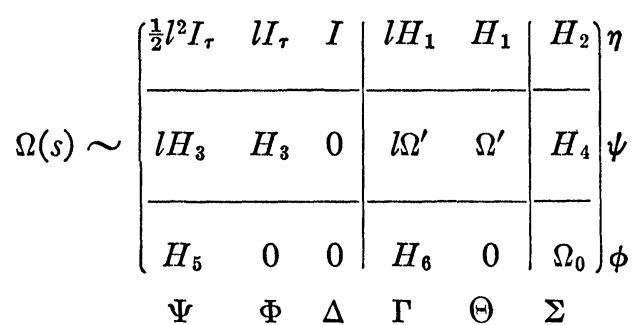

The proof of Proposition 14.36 follows more or less by inspection using the normalizations (14.33)-(14.35).

To put (14.37) in better form, we multiply $\Omega(s)$ on the left with 


$$
\frac{1}{l^{2}}\left(\begin{array}{ccc}
2 I_{\tau} & 0 & 0 \\
-H_{3} & l I_{\tilde{p}-p} & 0 \\
-H_{3} & 0 & l^{2} I_{h^{2,0}\left(V_{0}\right)}
\end{array}\right)
$$

to obtain the new period matrix

$$
\Omega_{1}(s) \sim\left(\begin{array}{cccccc}
I_{\tau} & 0 & 0 & 0 & 0 & 0 \\
0 & 0 & 0 & \Omega^{\prime} & 0 & 0 \\
0 & 0 & 0 & 0 & 0 & \Omega_{0}
\end{array}\right) .
$$

As a check on our computations, let us try to verify the 2 nd bilinear relation $\Omega(s) Q^{t}\{\Omega(s)\}^{*}>0(s \neq 0)$. From (14.32) and (14.38) we have

$$
\begin{aligned}
\Omega(s) Q^{t}\{\Omega(s)\}^{*}= & \\
& \left(\begin{array}{ccc}
\frac{1}{2}(\log |s| / \pi)^{2} I_{\tau} & * & * \\
* & (-\log |s| / \pi) i \Omega^{\prime} J t \bar{\Omega}^{\prime} & * \\
* & * & \Omega_{0} Q^{t} \bar{\Omega}_{0}
\end{array}\right)
\end{aligned}
$$

where the ${ }^{*}$ denotes lower order terms. Since $i \Omega^{\prime} J^{t} \bar{\Omega}^{\prime}=\operatorname{Im} Z^{\prime}>0$ by (14.19) and $\Omega_{0} Q_{0}^{t} \bar{\Omega}_{0}>0$ by the 2 nd bilinear relation for $\tilde{V}_{0}$, we indeed have $\Omega(s) Q^{t}\{\Omega(s)\}^{*}>0(s \neq 0)$.

(d) Some conclusions. We are not able to draw the same strong conclusions in this example as was done in $\$ 13$ for curves because the modular variety $D / \Gamma$ has not been compactified, or even partially compactified (cf. \$9), in the situation at hand. What we can say is given by Propositions (14.40) and (14.43) below.

(14.40). Proposition. Deligne's conjecture (9.17) is true "in the limit."

REMARK. Let me explain what this means. The Hodge filtration

$$
H^{2,0}\left(V_{s}\right) \subset H^{2,0}\left(V_{s}\right)+H^{1,1}\left(V_{s}\right)_{0} \subset H^{2}\left(V_{s}, \mathrm{C}\right)_{0}
$$

is to be thought of as a (many-valued) holomorphic filtration

$$
F^{0}(s) \subset F^{1}(s) \subset E \quad\left(F^{1}(s)=F^{0}(s)^{\perp}\right)
$$

of a fixed vector space $E\left(=H^{2}\left(V_{s_{0}}, C\right)_{0}\right)$ with the property that turning around $s=0$ changes $F^{p}(s)$ into $T \cdot F^{p}(s)$ where $T \in \operatorname{Aut}\left(H^{2}\left(V_{s_{0}}, \boldsymbol{C}\right)_{0}\right)$ is the P.-L. transformation. We write $s=\exp (2 \pi i t), t=\xi+i \eta$ where $\eta>0$, and consider $F^{p}(\exp (2 \pi i t))=F^{p}(t)$ as a single-valued holomorphic function of $t$ satisfying $F^{p}(t+1)=T \cdot F^{p}(t)$. Then we will find 
(cf. Proposition 14.43) that $F^{p}(0)=\lim _{\eta \rightarrow+\infty} F^{p}(\xi+i \eta)$ exists and gives a filtration $F^{0}(0) \subset F^{1}(0) \subset E$ of $E$. If we set

$$
F_{k}^{p}(0)=F^{p}(0) \cap W^{k} / F^{p}(0) \cap W^{k-1}
$$

where $W^{0} \subset W^{1} \subset W^{2} \subset W^{3} \subset W^{4}=E$ is the P.-L. filtration given by (9.12), then what we will prove is that (9.17) holds for the filtration $F_{k}^{0}(0) \subset F_{k}^{1}(0) \subset W^{k} / W^{k-1}$.

To state the second result, we lift the period mapping $\phi: \Delta^{*} \rightarrow D / \Gamma$ to

$$
\phi: H \rightarrow D, \quad t=\xi+i \eta \in H, \quad \phi(t+1)=\phi(t) \cdot T
$$

as above.

(14.43). Proposition. There exists a Siegel domain $\mathfrak{F} \subset D$ and an equivariant embedding $\psi: D^{\prime} \times D^{\prime \prime} \rightarrow \partial D$, where $D^{\prime}(\cong \operatorname{Sp}(n, R) / U(n))$ and $D^{\prime \prime}(\cong S O(2 h, k) / U(h) \times S O(k))$ are period matrix domains for 1-forms and 2-forms respectively, such that:

(i) the normalizer $N(F)=\{g \in G: g F=F\}$ and centralizer $Z(F)$ $=\{g \in G: g f=f$ for all $f \in F\}$ of $F=\psi\left(D^{\prime} \times D^{\prime \prime}\right)$ are $Q$-subgroups of $G$; (ii) if we restrict the period mapping (14.42) to the strip $\mathfrak{F}_{1}$ $=\left\{\xi+i \eta:|\xi| \leqq \frac{1}{2}, \eta \geqq \eta_{0}\right\}$, then $\phi\left(\Im_{1}\right) \subset F ;$ and

(iii) $\lim _{\eta \rightarrow+\infty} ; \xi+i \eta \in F_{1} \phi(t)=\phi(0)$ exists and is a point of $F \cap \mathcal{F}$.

Propositions (14.40) and (14.43) are proved using the asymptotic formulae (14.37) and (14.38) for the period matrix in the same way that Theorem (13.1)' and (9.17) (for $q=1$ ) followed from (13.6). The proof of (14.40) is completely straightforward, and the proof of (14.43) is also fairly direct once we have some information on Siegel domains in the period matrix space $D$. The requisite data on such Siegel domains can be gleaned from $\$ \$ 11,12$ of Borel's lecture notes Ensembles fondamentaux pour les groupes arithmetiques et formes automorphes, which are available from l'École Normale Supérieure, Paris. The computations are a little messy and not particularly enlightening so we will omit them.

15. Some remarks on and examples of the local invariant cycle problem. We want to give some comments about, and two examples of, the local invariant cycle problem (8.1).

(a) The first remark is that the local invariant problem should probably be stated for cohomology with rational coefficients. If this is done, then the problem behaves correctly with regard to taking ramified coverings of the parameter space.

Indeed, let 


$$
\begin{aligned}
\bar{Y}^{\prime} & \stackrel{\pi}{\rightarrow} \bar{Y} \\
f^{\prime} \downarrow & \downarrow f f \\
\Delta^{\prime} & \stackrel{\pi}{\rightarrow} \Delta
\end{aligned}
$$

be a diagram between local situations $f^{\prime}: \bar{Y}^{\prime} \rightarrow \Delta^{\prime}, f: \bar{Y} \rightarrow \Delta, V_{\boldsymbol{s}^{\prime}}=V_{\pi\left(\boldsymbol{s}^{\prime}\right)}$, as in $\S 8(\mathrm{a})$, and where $\Delta^{\prime} \rightarrow \Delta$ is a standard ramified covering given by $s^{\prime} \rightarrow\left(s^{\prime}\right)^{k}$ for some $k>0$. Fix $s_{0} \in \Delta$ and $s_{0}{ }^{\prime} \in \Delta^{\prime}$ with $\left(s_{0}^{\prime}\right)^{k}=s_{0} \neq 0$ and let $I \subset H^{q}\left(V_{s_{0}}, Q\right), I^{\prime} \subset H^{q}\left(V_{s_{0}}, Q\right)$ be the respective invariant part of the cohomology. Using the identification $H^{q}\left(V_{s_{0}}, \boldsymbol{Q}\right)=H^{q}\left(V_{s_{0}}, \boldsymbol{Q}\right)$ and that $T^{\prime}=T^{k}$ for the P.-L. transformations, we have $I \subset I^{\prime}$. If $\phi \in I$, and if there exists $\Phi \in H^{q}(\bar{Y}, Q)$ with $\Phi \mid V_{s_{0}}=\phi$, then $\Phi^{\prime}=\pi^{*} \Phi$ satisfies $\Phi^{\prime} \mid V_{s^{\prime} 0}=\phi$. Conversely, if $\phi \in I^{\prime}$, and if there is $\Phi^{\prime} \in H^{q}\left(\bar{Y}^{\prime}, Q\right)$ with $\Phi^{\prime} \mid V s_{s_{0}}=\phi$, then we may use the Gysin map (integration over the fibre) to define $\Phi=\pi_{*} \Phi^{\prime} \in H^{q}(\bar{Y}, Q)$ and

$$
\Phi \mid V_{s_{0}}=\sum_{\mu=0}^{k} T^{\mu} \phi
$$

This explains then how the invariant cycle problem behaves under ramified coverings of the base. In particular the problem is independent of the smooth compactification of $f: Y \rightarrow \Delta^{*}$.

(b) Taking $f: \bar{Y} \rightarrow \Delta$ as above and letting $h: V \rightarrow V_{0}$ be the collapsing map (defined up to isotopy) of a general fibre $V=V_{s_{0}}\left(s_{0} \neq 0\right)$ onto the singular fibre $V_{0}$ and $g: V_{s_{0}} \rightarrow V_{s_{0}}$ be the P.-L. homeomorphism, we see from $\S 3$ of Clemens' paper [14] that $h$ is a fibration of a very special sort, and furthermore we may assume that $h \circ g=h$. Using this Clemens, and also Grothendieck, analyzes the Leray spectral sequence of $h: V \rightarrow V_{0}$ in which the P.-L. transformation $g^{*}$ operates (Clemens uses homology). In this framework, we want to see what the local invariant cycle problem comes down to. In so doing we will also sketch a proof of the monodromy theorem 3.1.

Let $R_{h_{*}}^{q}=R_{h_{*}}^{q}(Q)$ be the Leray sheaf on $V_{0}$, which we recall comes from the presheaf $U \rightarrow H^{q}\left(h^{-1}(U), Q\right)$, where $U$ is an open set on $V_{0}$. Using (1.2) these sheaves are easily calculated as follows: If $x_{0} \in V_{0}$ is given locally by $z_{1}^{a_{1}} \cdots z_{k}^{a_{k}}=0$, then $h^{-1}\left(x_{0}\right)$ has the homotopy type of the disjoint union of g.c.d. $\left(a_{1}, \cdots, a_{k}\right)$ real tori of dimension $k-1$, and the stalk $\left(R_{h_{*}}^{q}\right)_{x_{0}}=H^{q}\left(h^{-1}\left(x_{0}\right), Q\right)$. The P.-L. transformation $g^{*}$ operates on the Leray sheaves $R_{h_{*}}^{q}$ (cf. $\S 4$ of [14]), and it follows from the above remark that $\left(g^{*}\right)^{N_{x}}=I$ on $\left(R_{h_{*}}^{a}\right)_{x}$, where $N_{x}$ is the 1.c.m. of $\left(a_{1}, \cdots, a_{k}\right)$ above.

Now we recall that there is a spectral sequence $\left\{E_{r}^{p . q}\right\}$ with the following properties: 
(i) $E_{2}^{p, q}=H^{p}\left(V_{0}, R_{h_{*}}^{q}\right)$, and

(ii) there is an increasing filtration $H^{r}(V, Q)_{0} \subset \cdots \subset H^{r}(V, Q)_{r}$ $=H^{r}(V, Q)$ of $H^{r}(V, Q)$ with the associated graded module being $E_{\infty}^{r, 0}+\cdots+E_{\infty}^{0, r}$.

Furthermore, the P.-L. transformations $g^{*}: R_{h_{*}}^{q} \rightarrow R_{h_{*}}^{q}$ induce linear maps $T_{r}: E_{r}^{p, q} \rightarrow E_{r}^{p, q}$ in the spectral sequence which commute with all the differentials $d_{r}: E_{r}^{p, q} \rightarrow E_{r}^{p+r, q-r+1}(r \geqq 2)$. The full P.-L. transformation $T: H^{r}(V, Q) \rightarrow H^{r}(V, Q)$ preserves the filtration on $H^{r}(V, Q)$ and induces $T_{\infty}$ on the associated graded $\sum_{p=0}^{r} E_{\infty}^{r-p, p}$.

From the above information we have that $T_{\infty}^{N}-I=0$ on $E_{\infty}^{r-p, p}$ for a suitable $N$. This gives us that

$$
T^{N}-I: H^{r}(V, Q)_{p} \rightarrow H^{r}(V, Q)_{p-1},
$$

from which the monodromy theorem follows.

The next approximation to $T^{N}$ is

$$
T^{N}-I: E_{\infty}^{r-p, p} \rightarrow E_{\infty}^{r-p+1, p-1}
$$

which is calculated using intersection numbers in Theorem 4.4 in [14].

The image of $H^{q}(\bar{Y}, Q) \rightarrow H^{q}\left(V_{s}, Q\right)$ is $E_{\infty}^{q, 0}$, and so the local invariant cycle problem is just the following:

(15.5). Reformulation of local inVARIANT CYCle Problem. Assuming (as we may by (a) above) that $T$ is already unipotent, prove that $E^{q, 0}=\operatorname{ker}(T-I)$.

(c) We will prove the

(15.6). Proposition. The local invariant cycle problem is true for curves.

Proof. We will use the following result of Mumford: Given a situation $f: Y \rightarrow \Delta^{*}$ where the fibres $V_{s}$ are all nonsingular curves, we may pass to a ramified covering $f^{\prime}: Y^{\prime} \rightarrow \Delta^{\prime *}$ and find a smooth compactification $f^{\prime}: \bar{Y}^{\prime} \rightarrow \Delta^{\prime}$ such that the singular curve $V_{0}$ has only normal crossings, and where all components of $V_{0}$ are smooth and have multiplicity one.

Let $p_{1}, \cdots, p_{n}$ be the double points of $V_{0}$. Then we have for the Leray sheaves that

$$
\begin{aligned}
& R_{h_{*}}^{0}=Q_{v_{0}}, \\
& R_{h_{*}}^{1}=Q_{p_{1}} \oplus \cdots \oplus Q_{p_{n}},
\end{aligned}
$$

where $Q_{v_{0}}$ is the constant sheaf $\boldsymbol{Q}$ on $V_{0}$ and $\boldsymbol{Q}_{p_{j}}$ is the "skyscraper sheaf" with stalk $\boldsymbol{Q}$ at $p_{j}$ and zero elsewhere. 
Let $C_{1}, \cdots, C_{m}$ be the irreducible components of $V_{0}$, and denote by $\pi(V), \pi\left(C_{j}\right)$ the genus of $V, C_{j}$ respectively. Then we have

$$
\pi(V)=\sum_{j=1}^{m} \pi\left(C_{j}\right)+n-m+1,
$$

so that $n-m+1$ is the rank of the vanishing cycle group, which is just the kernel of the surjective map $h_{*}: H_{1}(V, Q) \rightarrow H_{1}\left(V_{0}, Q\right)$.

Referring to (15.7), we have $E_{2}^{p, q}=0$ unless $q=0$ or $p=0$ and $q=1$. Thus the only nonzero differential in the spectral sequence is

$$
\begin{array}{rl}
d_{2}: E_{2}^{0,1} & \rightarrow E_{2}^{2,0} \\
\mathbb{R} & \mathbb{R} \\
Q_{p_{1}} \oplus \cdots \oplus \mathcal{Q}_{p_{n}} & \rightarrow \mathbb{Q}^{m}
\end{array}
$$

and it follows from (15.8) that $d_{2}$ in (15.9) has kernel isomorphic to $Q^{n-m+1}$. The filtration on $H^{1}(V, Q)$ has only two steps, and so is just the exact sequence

$$
0 \rightarrow H^{1}\left(V_{0}\right) \rightarrow H^{1}(V) \rightarrow Q^{n-m+1} \rightarrow 0,
$$

and it is easy to see that $T-I$ is injective on $H^{1}(V) / H^{1}\left(V_{0}\right) \cong Q^{n-m+1}$ using what we have said together with the "classical" P.-L. formula [7], which gives us the relation

$$
\operatorname{rank}\left\{(T-I) H_{1}(V)\right\}=\pi(V)-\sum_{j=1}^{n} \pi\left(C_{j}\right)=n-m+1 .
$$

(d) Essentially we were able to prove Proposition 15.6 by using Mumford's theorem to put us in a simple enough situation so that we could give a direct geometric discussion of the vanishing and invariant cycles. On the contrary, if we take even the simplest example of algebraic surfaces degenerating, then we are unable to prove the invariant cycle conjecture unless we use some transcendental methods. Here is an example, due to Landman, which illustrates our point.

(15.11). Proposition. Let $f: \bar{Y} \rightarrow \Delta$ be as above and assume that

(i) the general fibre $V$ is a nonsingular surface, and

(ii) the singular fibre $V_{0}=S_{1} \cup S_{2}$ where $S_{1}$ and $S_{2}$ are smooth surfaces which intersect transversally in an irreducible, nonsingular curve $C$. Then if $\phi \in H^{2}(V)$ is an invariant cohomology class, there exists $\Phi \in H^{2}(\bar{Y})$ such that $\Phi \mid V=\phi$.

Proof. We want to use (15.5). The Leray sheaves are given by 


$$
R_{h_{*}}^{0}=Q_{v_{0}}, \quad R_{h_{*}}^{1}=Q c .
$$

Thus $E_{2}^{p, q}=0$ if $q \geqq 2$ and $E_{3}=\cdots=E_{\infty}$. The differentials

$$
\begin{array}{clll}
d_{2}: E_{2}^{0,1} \rightarrow & E_{2}^{2,0}, & d_{2}: E_{2}^{2,1} & \rightarrow E_{2}^{4,0} \\
\| & \| & \| & \| \\
H^{0}(C) & H^{2}\left(V_{0}\right) & H^{2}(C) & H^{4}\left(V_{0}\right)
\end{array}
$$

turn out to be injective since $C$ is irreducible. The remaining differential

$$
\begin{array}{cl}
d_{2}: E_{2}^{1,1} & \rightarrow E_{2}^{3,0} \\
\| & \| \\
H^{1}(C) & H^{3}\left(V_{0}\right)
\end{array}
$$

is the essential one, and we claim that

(15.14). Lemma. In (15.13), we have

$$
H^{1}(C)=\left(\operatorname{ker} d_{2}\right) \oplus\left(\operatorname{ker} d_{2}\right)^{\perp}
$$

as an orthogonal direct sum over $\boldsymbol{Q}$, and where the pairing on $H^{1}(C)$ is the cup product.

Proof. From the Mayer-Vietoris sequence

$$
H^{2}\left(S_{1}\right) \oplus H^{2}\left(S_{2}\right) \stackrel{r}{\rightarrow} H^{2}(C) \rightarrow H^{3}\left(V_{0}\right) \rightarrow H^{3}\left(S_{1}\right) \oplus H^{3}\left(S_{2}\right) \rightarrow 0,
$$

and the fact that $r$ is onto, we see that ker $d_{2}$ is the same as the kernel of

$$
H^{1}(C) \rightarrow H^{3}\left(S_{1}\right) \oplus H^{3}\left(S_{2}\right) .
$$

Now (15.15) is derived from the mapping of Albanese varieties

$$
\operatorname{Alb}(C) \rightarrow \operatorname{Alb}\left(S_{1}\right) \oplus \operatorname{Alb}\left(S_{2}\right),
$$

and Lemma 15.14 follows from the Poincaré complete reducibulity theorem.

(15.16). Remark. From the proof we see that (15.14) is not a topological fact, but depends on having Kähler manifolds.

We may now complete the proof of (15.11). The filtration on $H^{2}(V)$ induced from the spectral sequence is again just the exact sequence

$$
0 \rightarrow E_{\infty}^{2,0} \rightarrow H^{2}(V) \rightarrow\left\{\operatorname{ker} d_{2}\right\} \rightarrow 0 .
$$


To see that $T-I: \operatorname{ker} d_{2} \rightarrow H^{2}(V)$ is injective, we consider the diagram

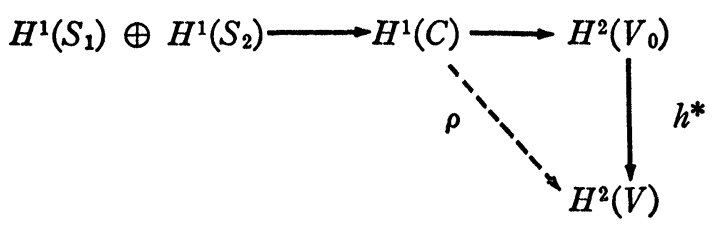

where the horizontal row is a piece of the Mayer-Vietoris sequence. According to the proof of Lemma 15.14, $\rho$ is zero on $\left(\operatorname{ker} d_{2}\right)^{\perp}$, and our result follows from Lemma 15.14 and Clemens' formula 5.1 in [14], which in our special case is just:

$$
(T-I) \psi \wedge \rho(\theta)[V]= \pm \psi \wedge \theta[C],
$$

for $\psi, \theta \in$ ker $d_{2}$.

APPENDix. Recent PROGRESS ON THE PROBLEMS AND CONJECTURES

(i) In the introduction I listed hyperbolic complex analysis and the theory of algebraic Lie groups as being the main tools used thus far in studying periods of integrals. To this I should like to add the algebraicizing of cohomology and resulting technique of reduction modulo $p$ of the (algebraic) de Rham cohomology as a method which has led to significant progress in the study of periods. A hint of this was given in $\$ 4$ where Katz's proof of the regularity of the Picard-Fuchs equations was outlined. It was also pointed out there that this argument also gives the eigenvalue part of the monodromy theorem 3.1, but had not yet yielded the elementary divisor part of that theorem. Just recently Katz, with some valuable suggestions from Deligne, has succeeded in extending his techniques so that he gets out not only the full monodromy theorem 3.1 , but also obtains the refinement on the elementary divisors predicted by (8.4). In summary then, this method now gives a unified treatment of the regularity Theorem 4.3 and the monodromy theorem 3.1 in the strong form discussed in Conjecture 8.4, and so this technique must certainly now be listed as among the main tools used in the study of periods.

(ii) In Problem 7.3 the question of the global Torelli theorem for nonsingular cubic threefolds was discussed. There has recently been progress on this problem in a joint work of $\mathrm{H}$. Clemens and the author, and it seems now quite likely that the conjecture will go through as stated.

(iii) In $\S 8$ I gave a tentative outline of how Conjecture 8.4 might 
be approached group-theoretically. This discussion was somewhat off target, especially the second remark following 8.4, and I should like to add a few comments here.

First of all, it was pointed out to me by Borel and Schmid that the statement about the number of simple factors of $H$ controlling the index of unipotency of the P.-L. transformation was not too good, and they suggested how the group theoretic version of the conjecture might be phrased. Following the notation and terminology given in (c) of $\S 4$, we should therefore like to revise (8.4) to read

ConjeCture $8.4^{\prime}$. Let $\Phi: H \rightarrow D$ be a horizontal holomorphic mapping which satisfies $\Phi(z+1)=\Phi(z) \cdot T$ for some $T \in G_{Z}$ (this is the group of integral points in $G)$. We assume that $T$ is of infinite order and let

$$
\Omega_{0}=\lim _{\operatorname{Im}(z) \rightarrow \infty} \Phi(z)
$$

be the point in $\partial D \subset \check{D}$ as given in Theorem 4.11. Let $B\left(\Omega_{0}\right) \subset \check{G}$ be the stability group of $\Omega_{0} \in \check{D}=B \backslash \breve{G}$, so that $B\left(\Omega_{0}\right)$ is a parabolic subgroup of $\breve{G}$ which contains $T$. The conjecture is that a suitable power $T^{N}$ belongs to the unipotent radical $R_{u}\left(B\left(\Omega_{0}\right)\right)$.

REMARKs. This conjecture would yield the monodromy theorem 3.1 in the strong form conjectured by 8.4. Also, the proof would almost certainly give fairly good information on the behavior of the period matrix $\Phi(z)$ as $\operatorname{Im}(z) \rightarrow \infty$.

It should be pointed out that the monodromy theorem (in the strong form 8.4) does not conversely imply the above Conjecture $8.4^{\prime}$, because a statement about the index of unipotency of $T$ is not enough to put $T$ in $R_{u}\left(B\left(\Omega_{0}\right)\right)$. Therefore, it is worth remarking that in case $\Phi: H \rightarrow D$ arises from an algebraic family of algebraic varieties and $q=1$ or 2 , then it is in fact the case that $T \in R_{u}\left(B\left(\Omega_{0}\right)\right.$ ) (c.f. just below (13.26) in $\$ 13$ and (14.32)).

(iv) Problem 12.1 has been partially answered in that it can be proved that every point $w \in W$ has a Zariski neighborhood $U$ whose universal covering $\widetilde{U}$ is complex analytically isomorphic to a bounded domain in $C$. The main question now seems to be to somehow rigidify the embedding $\tilde{U} \subset \subset C^{n}$ so that it is as "nice" a domain as possible.

(v) There are two additions to the bibliography which I would like to mention. The first is a manuscript Die Monodromie der Isolierten Singularitäten von Hyperflächen by E. Brieskorn which gives a thorough discussion of the de Rham cohomology and monodromy associated to an isolated singular point on a complex analytic hypersurface. The second is the recent paper The action of a real semisimple group 
on a complex flag manifold. I: orbit structure and holomorphic arc components, Bull. Amer. Math Soc. 75 (1969), 1121-1237, by J. A. Wolf in which he gives the beginning of a general discussion about homogeneous complex manifolds among which our period matrix domains are a special case.

\section{REFERENCES}

1. P. Deligne, Théorie de Hodge (to appear). (Cf. Deligne's article Théorème de Lefschetz et critères de dégénérescence de suites spectrales, Inst. Hautes Etudes Sci. Publ. Math. No. 35 (1968), 107-126.)

2. P. Griffiths, Periods of integrals on algebraic manifolds. I, II, Amer. J. Math. 90 (1968), 568-626; 805-865. MR 37 \#5215; MR 38 \#2146.

3. - On the periods of integrals on algebraic manifolds, Rice Univ. Studies 54 (1968), 21-38.

4. P. Griffiths and W.Schmid, Locally homogeneous complex manifolds, Acta Math. (to appear).

5. A. Grothendieck, On the de Rham cohomology of algebraic varieties, Inst. Hautes Etudes Sci. Publ. Math. No. 29 (1966), 95-103. MR 33 \#7343.

6. W. V. D. Hodge, The theory and applications of harmonic integrals, 2nd ed., Cambridge Univ. Press, New York, 1952. MR 14, 500.

7. S. Lefschetz, L'analysis situs et la géometrie algébrique, Gauthier-Villars, Paris, 1924.

8. J. Milnor, Singular points of complex hypersurfaces, Ann. of Math. Studies, no. 61, Princeton Univ. Press, Princeton, N. J., 1968.

9. A. Weil, Introduction a l'étude des variêtés kählériennes, Publ. Inst. Math. Nancago, no. VI, Actualités Sci. Indust., no. 1267, Hermann, Paris, 1958. MR 22 \#1921.

10. W. L. Baily, Jr. and A. Borel, Compactification of arithmetic quotients of bounded symmetric domains, Ann. of Math. (2) 84 (1966), 442-528. MR 35 \#6870.

11. A. Borel and R. Narasimhan, Uniqueness conditions for certain holomorphic mappings, Invent. Math. 2 (1967), 247-255. MR 35 \#414.

12. S. S. Chern, Characteristic classes of Hermitian manifolds, Ann. of Math. (2) 47 (1946), 85-121. MR 7, 470.

13. - On holomorphic mappings of Hermitian manifolds of the same dimension, Proc. Sympos. Pure Math., vol. 11, Amer. Math. Soc., Providence, R. I., 1968, pp. 157-170. MR 38 \#2714.

14. C. H. Clemens, Jr., Picard-Lefschetz theorem for families of nonsingular algebraic varieties acquiring ordinary singularities, Trans. Amer. Math. Soc. 136 (1969), 93-108. MR 38 \#2135.

15. P. Deligne, Monograph on connexions with regular singular points, SpringerVerlag (to appear).

16. P. Griffiths, Monodromy of homology and periods of integrals on algebraic manifolds, preprint from Princeton University, Princeton, N. J.

17. - A theorem on periods of integrals on algebraic manifolds, Rice Univ. Studies (to appear).

18. A. Grothendieck, Un théorème sur les homomorphismes de schémas abéliens, Invent. Math. 2 (1966), 59-78. MR 36 \#180.

19. P. Deligne and A. Grothendieck, S. G. A. 7 (on monodromy theorems), Inst. Hautes Études Sci. Publ. Math. (1968/70). 
20. K. Kodaira, On compact analytic surfaces. III, Ann. of Math. (2) 78 (1963), 1-40. MR $32 \# 1730$.

21. - On characteristic systems of families of surfaces with ordinary singularities in a projective space, Amer. J. Math. 87 (1965), 227-256. MR 31 \#652.

22. A. Landman, On the Picard-Lefschetz transformations, Thesis, University of California, Berkeley, Calif., 1966.

23. D. Lieberman, Higher Picard varieties, Amer. J. Math. 90 (1968), 1165-1199.

24. W. Schmid, On a conjecture of Langlands, Ann. of Math. (to appear).

25. Séminaire H. Cartan, Fonctions automorphes, 10e Année, 1957/58, Secrétariat mathématique, Paris, 1958. MR $21 \# 2750$.

26. H. Wu, Normal families of holomorphic mappings, Acta Math. 119 (1967), 193-233. MR 37 \#468.

Princeton University, Princeton, New Jersey 08540 and

Institute des Hautes Études Scientifiques, Bures-sur-Yvette, France 